\title{
Climate Science in the Tropics: Waves, Vortices, and PDEs
}

\author{
Boualem Khouider ${ }^{1}$, Andrew J Majda ${ }^{2}$ and Samuel N \\ Stechmann $^{3}$ \\ ${ }^{1}$ Department of Mathematics and Statistics, University of Victoria, PO BOX 3045 \\ STN CSC Victoria, B.C. Canada V8W 3P4 \\ E-mail: khouider@uvic.ca \\ ${ }^{2}$ Department of Mathematics, and Center for Atmosphere-Ocean Science, Courant \\ Institute of Mathematical Sciences, New York University, 251 Mercer Street, New \\ York, NY 10012 USA \\ ${ }^{3}$ Department of Mathematics, and Department of Atmospheric and Oceanic Sciences, \\ University of Wisconsin-Madison, 480 Lincoln Drive, Madison, WI 53706 USA
}

\begin{abstract}
.
Clouds in the tropics can organize the circulation on planetary scales and profoundly impact long range seasonal forecasting and climate on the entire globe, yet contemporary operational computer models are often deficient in representing these phenomena. On the other hand, contemporary observations reveal remarkably complex coherent waves and vortices in the tropics interacting across a bewildering range of scales from kilometers to ten thousand kilometers. This paper reviews the interdisciplinary contributions over the last decade through the modus operandi of applied mathematics to these important scientific problems. Novel physical phenomena, new multi-scale equations, novel PDEs, and numerical algorithms are presented here with the goal of attracting mathematicians and physicists to this exciting research area.
\end{abstract}

PACS numbers: 00.00, 20.00, 42.10

Keywords: Climate science, Tropical meteorology, waves, vortices, convection, PDEs, MJO, GCMs, tropical waves, moisture, clouds, cumulus parameterization

Submitted to: Nonlinearity 


\section{Introduction}

One of the grand challenges of contemporary science is a comprehensive predictive model for the atmosphere and coupled climate system. This is one of the most difficult multiscale problems in contemporary science because there is an incredible range of strongly interacting anisotropic nonlinear processes over many spatio-temporal scales; contemporary comprehensive computer models, called general circulation models (GCMs), are currently incapable of adequately resolving or parameterizing many of these interactions on time scales appropriate for seasonal prediction as well as climate change projections. An overview can be found in [1].

Basic questions which drive climate research are the prediction of the weather from 1 to 14 days, the prediction of climate variations on seasonal to yearly time scales and finally, climate change projections on decadal and centennial time scales as well as quantifying the uncertainty associated with these predictions. One of the striking recent observational discoveries is the profound impact of variations in the tropics on all of these problems. The primary issue in the influence of the tropics occurs through the interaction and organization of clouds into clusters, super clusters, and planetary scale dynamics, an inherently fully nonlinear multiscale process. For climate change, water vapor is arguably the most important greenhouse gas, and the microphysical processes in clouds are a key mechanism for radiative feedback. In fact, only a $4 \%$ change in average cloudiness would overwhelm the effects of CO2 in climate change.

The dominant component of intraseasonal variability in the tropics is the 40- to 50day tropical intraseasonal oscillation, often called the Madden-Julian oscillation (MJO) after its discoverers $[2,3]$. In the troposphere, the MJO is an equatorial planetaryscale wave envelope of complex multiscale convective processes. It begins as a standing wave in the Indian Ocean and propagates eastward across the western Pacific Ocean at a speed of roughly $5 \mathrm{~m} \mathrm{~s}^{-1}$ [4]. The planetary scale circulation anomalies associated with the MJO significantly affect monsoon development, intraseasonal predictability in mid-latitudes, and the development of the El Nino Southern Oscillation (ENSO) in the Pacific Ocean, which is one of the most important components of seasonal prediction $[4,5]$.

Despite the widespread importance of the MJO, present day computer GCMs typically have poor representations of it [6]. A growing body of evidence suggests that this poor performance of GCMs is due to the inadequate treatment of interactions of organized tropical convection on multiple spatio-temporal scales [6, 7]. Such hierarchical organized structures that generate the MJO as their envelope are the focus of current observational initiatives and modeling studies [7], and there is a general lack of theoretical understanding of these processes and the MJO itself.

Over the last decade, the modus operandi of modern applied mathematics, blending novel asymptotic, qualitative, and numerical modelling as well as rigorous analysis, has been utilized to address many of the pressing issues in climate science described above. This work is inherently interdisciplinary and driven by the attempts to develop theories 
which predict the complex observational record and new algorithms to improve GCMs. Therefore, many of the papers with new perspectives and results from modern applied mathematics and cited in this review are published in disciplinary journals such as the Journal of the Atmospheric Sciences, the Quarterly Journal of the Royal Meteorological Society, or the Journal of Climate. Our goal in this review article is to make this exciting and important area of interdisciplinary research accessible to a wide range of mathematicians and physicists while also illustrating this interdisciplinary research perspective. We hope the range of new phenomena, methods, and results presented here inspires many more mathematicians and physicists to work in this important and exciting research area. The remainder of this paper has the following table of contents.

\section{Contents}

1 Introduction $\quad 2$

2 Tropical weather and climate $\quad 4$

2.1 Climatology . . . . . . . . . . . . . . . . . . . . 4

2.2 Waves and vortices . . . . . . . . . . . . . . . . 5

$\begin{array}{lll}3 & \text { Fluid dynamics of the tropical atmosphere } & 7\end{array}$

3.1 Dry dynamics . . . . . . . . . . . . . . . . . . . . 9 9

3.1.1 The barotropic-first baroclinic mode interaction system . . . . . . 9

3.1.2 Linear wave solutions and meridional structure . . . . . . . . . . . 10

3.1.3 Weakly nonlinear Kelvin waves . . . . . . . . . . . . . . . . 14

3.1.4 Nonlinear interaction of first and second baroclinic modes . . . . . 15

3.2 Moist dynamics . . . . . . . . . . . . . . . . . . . . . . . 19

3.2.1 The large-scale moisture equation . . . . . . . . . . . . . . . . . . 19

3.2.2 Simple one-heating mode model . . . . . . . . . . . . . . . . . . . 21

3.2.3 Limiting dynamic moisture front solutions . . . . . . . . . . . . 22

3.2.4 Analytic formulation of moisture front solution with finite

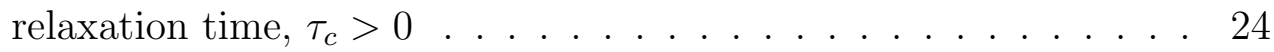

3.2.5 Wave reflection and transmission at the interface of a moisture front 26

4 Scale-dependent and multiscale models $\quad 29$

4.1 Two examples . . . . . . . . . . . . . . . . . . . . . . . . . . . . . . . . . 29

4.2 "Self-similarity" in tropical convection . . . . . . . . . . . . . . . . . 32

4.3 From planetary to sub-cloud scales . . . . . . . . . . . . . . . . 33

4.3.1 Moist dynamics on planetary scales . . . . . . . . . . . 33

4.3.2 Convectively coupled waves and convective momentum transport 33

4.3 .3 Superrotation . . . . . . . . . . . . . . . . 34

4.3.4 Superparameterization . . . . . . . . . . . . . . 34

4.3.5 Hurricane embryo . . . . . . . . . . . . . . . . . 35

4.3.6 Squall lines . . . . . . . . . . . . . . . . . 35 
4.3.7 Moist thermodynamics and mesoscale convection . . . . . . . 36

4.3.8 Shallow and deep cumulus clouds . . . . . . . . . . . . . 36

4.4 MJO skeleton model . . . . . . . . . . . . . . . . . . . . . . 37

5 Multicloud and multiscale models $\quad 41$

5.1 The multicloud model . . . . . . . . . . . . . . . . . . . . . 41

5.2 Convectively coupled equatorial waves in the multicloud model . . . . . . 43

5.3 The MJO analog wave . . . . . . . . . . . . . . . . 47

5.4 GCM simulation of the MJO and convectively coupled waves . . . . . . 48

5.5 Convective momentum transport and multiscale waves within the MJO . 50

5.6 A multiscale model for the MJO: the muscle . . . . . . . . . . . . . 53

6 Tropical-extratropical interactions $\quad 56$

6.1 Nonlinear resonance of longwave barotropic and baroclinic Rossby waves 57

6.2 Barotropic response to a slowly moving Kelvin wave . . . . . . . . . . . 59

6.3 Resonant interactions of equatorial waves and the diurnal cycle . . . . . 63

6.4 Extra-tropical initiation of the MJO . . . . . . . . . . . 66

7 Applied math and rigorous PDEs for the Tropics 68

7.1 Precipitation fronts . . . . . . . . . . . . . . . 68

7.2 Rigorous multiscale singular limits . . . . . . . . . . . . . . 70

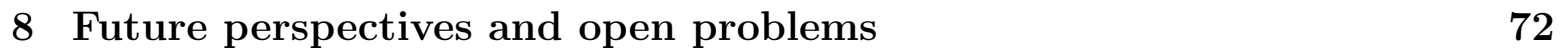

\section{Tropical weather and climate}

\subsection{Climatology}

The climatological mean state in the tropics includes two prominent circulation cells: one aligned in the north-south direction (the Hadley cell) and the other aligned in the east-west direction (the Walker cell). Figure 1 shows observations and a schematic diagram of these circulation cells, each of which consists of a moist cloudy region with ascending air and a dry desert region with descending air. Hence these cells demarcate the mean patterns of tropical convection, which tend to be roughly aligned with patterns of sea surface temperature (SST). For instance, the ascending region of the Walker cell occurs over the western Pacific Ocean and Indonesian maritime continent, where some of the warmest SSTs in the world are located; and the descending region of the Walker cell occurs over the eastern Pacific Ocean, where some of the coldest SSTs in the tropics are located. To summarize, the take-home message here is that the circulation is intimately related to the hydrological cycle and to deep convection in particular. A final indication of this can be seen by computing the north-south circulation in different ways (see figure 1a,b): the circulation is much stronger when computed in a way that accounts for moisture [8]. 
Computational models called GCMs are used to simulate the global atmospheric evolution. While GCMs capture many features realistically, they also struggle to represent some aspects of the tropical mean climate. One well-known example of this is the "double ITCZ problem," which can be crudely described as a tendency of some GCMs to produce two Intertropical Convergence Zones (ITCZs), one on each side of the equator, rather than a single ITCZ (see $[9,10]$ and references therein). Since the ITCZ patterns clouds and convection, it is believed that the double ITCZ problem is related to inadequate simulation of the relationships between circulation and the hydrological cycle (which includes a very large number of interactions, including atmosphere-ocean interactions).

As a final note, while it is tempting to treat the mean climate as an isolated entity, it would be cursory to try to fully understand the mean state without regard to the fluctuations.

\subsection{Waves and vortices}

Fluctuations about the mean state include a variety of waves and vortices. As was the case for the climatological mean state, a key feature of the waves and vortices in the tropics is that they are strongly coupled with water vapor and convection.

Tropical atmospheric waves, coupled with convection, occur on a wide variety of different length and time scales, as shown in figure 2. The left plot shows precipitation along the equator evolving in time for one year, and many waves can be seen propagating in the zonal ( $x$, east-west) direction. Using a Fourier transform in both space and time, the power spectrum of such precipitation data can be displayed as in the right plot (after removing a background power spectrum). A striking feature here is that peaks in spectral power are aligned with the dispersion curves of the (dry) linear equatorial shallow water equations. However, the dispersion curves are the only feature in common between the convectively coupled equatorial waves (CCEWs) and the dry linear waves: the structures of the CCEWs and the dry linear waves do not agree, and more complicated physics is needed to explain the CCEWs (see section 5.2 below). In addition to the CCEWs, there is also a low-frequency spectral peak that is not aligned with the dry linear dispersion curves. This is due to the MJO, which appears on time scales of 30-60 days and spatial scales of 10,000-40,000 km (zonal wavenumbers 1-4).

This pair of wave types - CCEWs and the lower-frequency MJO - is one example of the multiscale organization of tropical convection. As can be seen in the left plot of figure 2, the MJO is an eastward-propagating envelope (denoted by thin white lines) of smaller-scale convection and wave activity, including CCEWs. As other examples, CCEWs are, in turn, propagating envelopes of smaller-scale cloud systems, and the MJO envelope also modulates the occurrence of hurricanes and tropical cyclones, the most intense example of vortices in the tropics. It remains a difficult challenge to understand the interactions in this hierarchy of organized tropical convection, and one can no doubt see that multiscale models should play an important theoretical role in this area. 

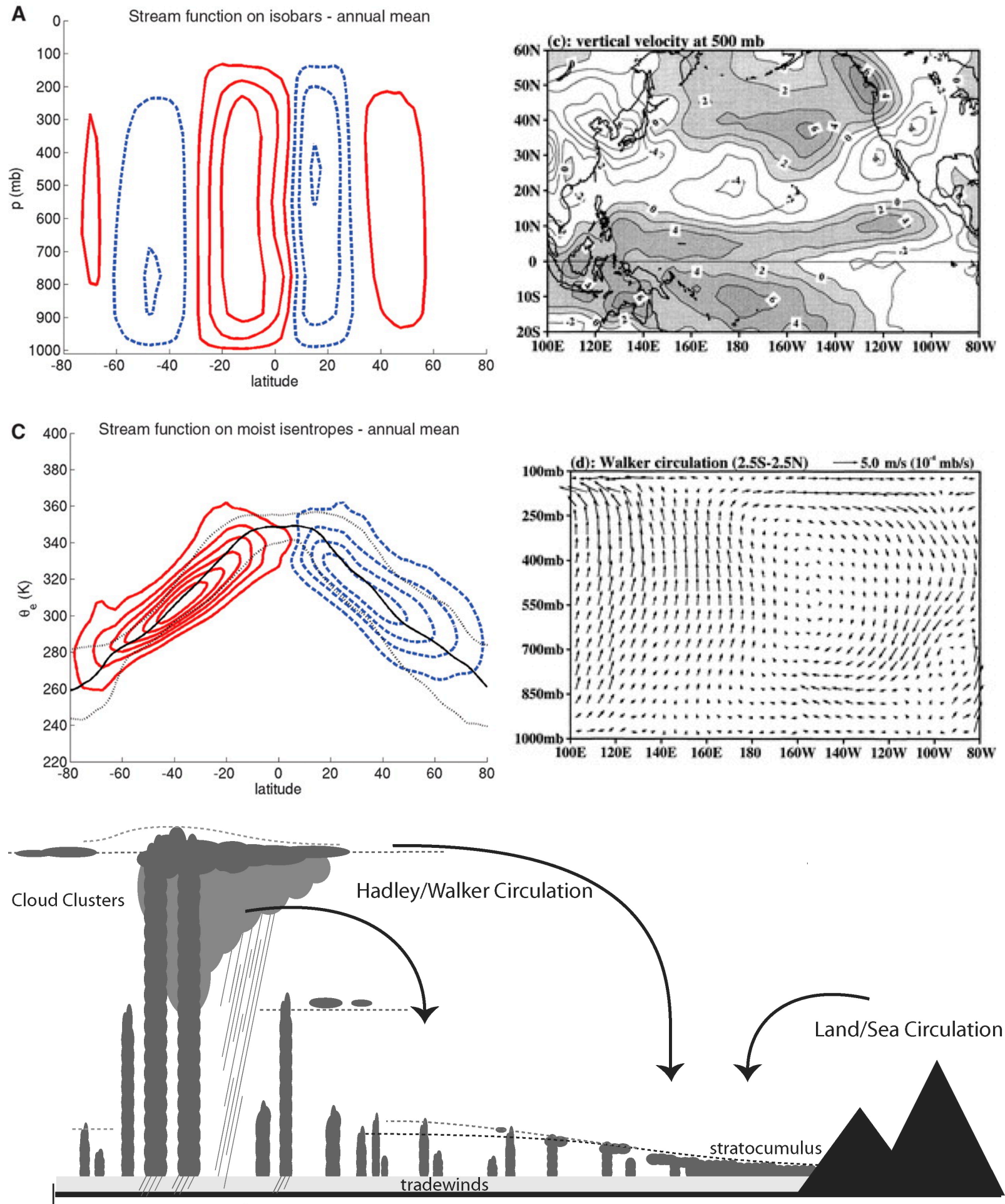

EQ warm, western tropical oceans

cold, eastern subtropical ocean

Figure 1. Time-averaged mean state of the tropics. (a) Stream function, averaged over longitude on surfaces of constant pressure. The two cells of the Hadley circulation extend from the equator to $\pm 30^{\circ}$ latitude. (b) Same as (a), but averages taken on surfaces of constant moist entropy. Two cells of the global circulation now extend from equator to pole, and the circulation appears stronger than in (a). From [8]. (c) Vertical velocity in the mid-troposphere in January. (d) The Walker circulation. Zonal-vertical velocity vectors, averaged from $2.5^{\circ} \mathrm{S}-2.5^{\circ} \mathrm{N}$. From [11]. (e) Schematic diagram of the cloud regimes associated with different branches of the Walker or Hadley circulations. From [12]. 


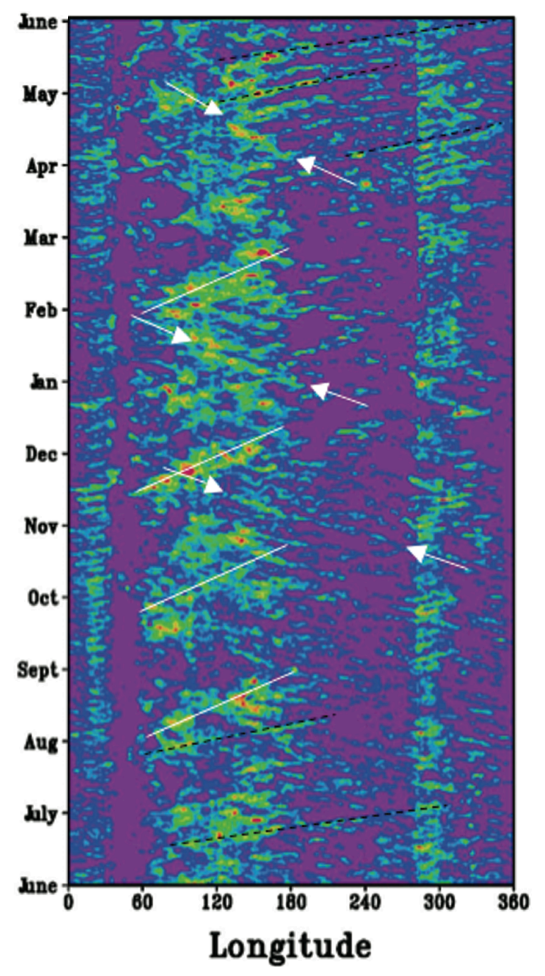

a) Symmetric CLAUS $T_{b}$ Spectrum

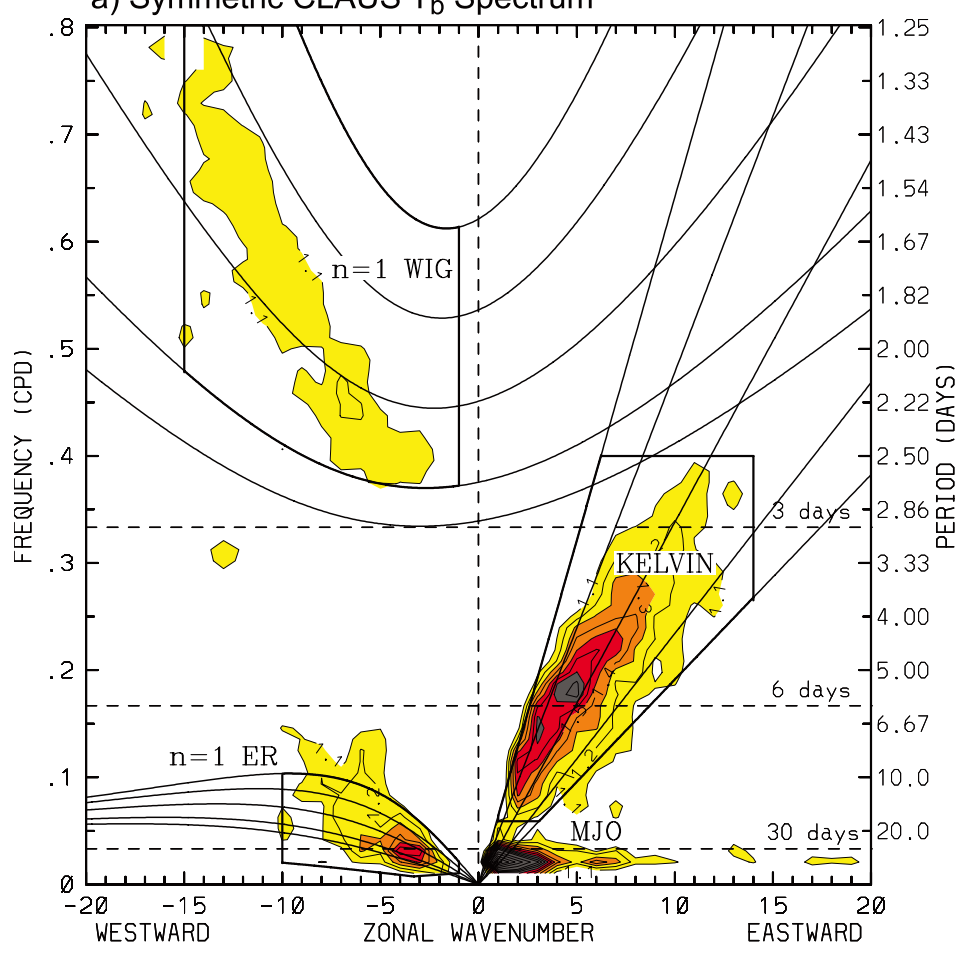

Figure 2. Fluctuations in clouds and precipitation near the equator. Left: Precipitation at the equator over one year. Adapted from [4]. Right: Fourier power spectrum of equatorial cloudiness (with a background spectrum removed), showing spectral peaks associated with convectively coupled equatorial waves and the MaddenJulian Oscillation. From [21].

For GCMs, the MJO and CCEWs are some of the most difficult atmospheric features to simulate realistically $[13,14,6,15]$. It is believed that this stems from an inadequate treatment of the multiscale hierarchy of tropical convection [7], and, in fact, some non-traditional approaches appear to have more success by accounting for this hierarchy in various ways $[16,17,18,19,20]$. One of the great difficulties here is that much of the convection and cloud systems are actually on subgrid-scales for longterm climate simulations with grid spacings of roughly $100 \mathrm{~km}$. It is hoped that GCM simulations will improve in tandem with advances in theoretical understanding, some of which is described below.

\section{Fluid dynamics of the tropical atmosphere}

Because of the vanishing Coriolis force at the equator, the equatorial atmosphere harbors a large spectrum of waves. These waves have a very complex vertical and meridional structure among which we distinguish barotropic waves that have the capability to travel poleward and baroclinic waves that are trapped in the vicinity of and propagate along the equator. In atmosphere and ocean sciences, the large scale flow is uniquely decomposed into its barotropic, i.e, independent of height, and baroclinic components. 
To illustrate these complex dynamics of the equatorial atmosphere, we consider the non-linear primitive equations, on the equatorial beta-plane; these are a special approximation of the Navier-Stokes equations on a rotating sphere where 1) density variations are ignored except in the buoyancy term; this is known as the Boussinesq approximation, 2) the vertical velocity variations are ignored in the vertical momentum equation, and 3) Earth's curvature is approximated by using a domain reduced to a periodic strip aligned along and centered at the equator. Assumption 2) results from scale analysis because, on the scales relevant for weather and climate predictions, there is a large discrepancy between the horizontal scales measured in thousands of kilometers and the vertical scales that are limited by the height of the troposphere of roughly 16 $\mathrm{km}$.

$$
\begin{aligned}
& \frac{\partial \mathbf{V}}{\partial t}+\mathbf{V} \cdot \nabla \mathbf{V}+W \frac{\partial \mathbf{V}}{\partial z}+\beta y \mathbf{V}^{\perp}=-\nabla \Phi+S_{\mathbf{V}}, \\
& \frac{\partial \Phi}{\partial z}=\frac{g \Theta}{\theta_{0}}, \\
& \frac{\partial \Theta}{\partial t}+\mathbf{V} \cdot \nabla \Theta+W \frac{\partial \Theta}{\partial z}+\frac{N^{2} \theta_{0}}{g} W=S_{\Theta}, \\
& \nabla \cdot \mathbf{V}+\frac{\partial W}{\partial z}=0 .
\end{aligned}
$$

Here $\mathbf{V}=(U, V)^{T}$ is the horizontal velocity field, where $U$ is the zonal (along the equator) component and $V$ is the meridional (North-South) component, and $W$ is the vertical velocity. The positive directions are from West to East, South to North, and from bottom to top, respectively. The scalars $\Phi$ and $\Theta$ are the pressure and potential temperature (i.e., the temperature that a parcel of air would have if displaced adiabatically in the vertical from its original position to a fixed reference position) perturbations, respectively. The total potential temperature, including a background that depends only on height, is given by

$$
\Theta^{\text {total }}(x, y, z, t)=\theta_{0}+\bar{\theta}(z)+\Theta(x, y, z, t)
$$

where $\theta_{0} \approx 300$ Kelvin is a reference constant temperature and $\bar{\theta}$ defines the vertical profile-background stratification and satisfies $N^{2}=\frac{g}{\theta_{0}} \frac{\partial \bar{\theta}}{\partial z}>0$ where $N$ is known as the Brunt-Väisälä buoyancy frequency; it is the frequency with which a vertically displaced parcel would oscillate around its equilibrium position under the influence of buoyancy if no other forces were present, in a stably stratified fluid. Here $x, y, z$ are respectively the zonal, meridional, and vertical coordinates and $t>0$ is time. We fix $z=0$ at the surface or bottom of the troposphere and $z=H$ is at the top of the troposphere $(H=16 \mathrm{~km})$ while $y$ measures the distance from the equator.

The first equation in (3.1) represents the conservation of horizontal momentum, where $\nabla=\left(\partial_{x}, \partial_{y}\right)^{T}$ is the horizontal gradient and $\mathbf{V}^{\perp}=(-V, U)^{T}$ with $\beta=2.28 \times 10^{-11}$ $\mathrm{m}^{-1} \mathrm{~s}^{-1}$ is the gradient of the Coriolis parameter at the equator. The second equation expresses hydrostatic balance, and the third one expresses conservation of potential temperature. Finally, the last one is the divergence free constraint derived from the continuity (conservation of mass) equation due to the Boussinesq approximation. The 
equations in (3.1) are known as the hydrostatic Boussinesq primitive equations. The terms $S_{\mathbf{V}}$ and $S_{\Theta}$ are the sources and sinks of momentum and potential temperature, respectively. In particular, $S_{\Theta}$ represents convective heating from clouds and radiative cooling (and heating), which constitute the main sources of energy input in the tropics.

The primitive equations are supplemented with the rigid boundary conditions

$$
\left.W\right|_{z=0} ^{z=H}=0
$$

\subsection{Dry dynamics}

To elucidate the dry dynamics of the tropical atmosphere, we first decompose the primitive equations (3.1) into their barotropic and baroclinic parts, following [22]; see also $[23,24,25,26]$. To do so, we invoke the normal modes of vertical structure associated with the linearized primitive equations (about the imposed background rest state), i.e., the linear operator part of (3.1). Because of the rigid lid boundary conditions in (3.2), the vertical velocity and potential temperature are expanded in terms of $\{\sin (m \pi z / H)\}_{m=1}^{\infty}$ while the horizontal velocities and pressure are expanded in terms of $\{\cos (m \pi z / H)\}_{m=0}^{\infty}$. The interested reader is referred to [22] for details. We note that $m=0$ corresponds to the barotropic mode that carries only a horizontal velocity $\overline{\mathbf{v}}=(\bar{u}, \bar{v})^{T}$ and pressure $\bar{p}$ and is independent of height while $m=1,2, \cdots$ define an infinite number of baroclinic modes that oscillate in the vertical and carry nontrivial vertical velocity and potential temperature components.

To derive the nonlinear dynamic equations for the barotropic and baroclinic flow components, we introduce the $L_{2}$ inner product in the vertical direction

$$
(f, g)=\frac{1}{H} \int_{0}^{H} f(z) g(z) d z
$$

3.1.1. The barotropic-first baroclinic mode interaction system We impose the following ansatz of truncating the primitive equations in the vertical direction onto the barotropic and first baroclinic modes:

$$
\left(\begin{array}{l}
\mathbf{V} \\
\Phi
\end{array}\right)(x, y, z, t)=\left(\begin{array}{c}
\overline{\mathbf{v}} \\
\bar{p}
\end{array}\right)(x, y, t)+\left(\begin{array}{c}
\mathbf{v}_{1} \\
p_{1}
\end{array}\right)(x, y, t) \sqrt{2} \cos (\pi z / H)
$$

and

$$
\left(\begin{array}{c}
W \\
\Theta
\end{array}\right)(x, y, z, t)=\left(\begin{array}{c}
w_{1} \\
\theta_{1}
\end{array}\right)(x, y, t) \sqrt{2} \sin (\pi z / H) .
$$

The $\sqrt{2}$ in front of the sine and cosine functions are added to obtain an orthonormal basis in the given $L_{2}$ inner product.

The hydrostatic balance equation yields the relationships $p_{1}=-\left(H g / \pi \theta_{0}\right) \theta_{1}$ while the divergence equation leads to $w_{1}=-(H / \pi) \nabla \cdot \mathbf{v}_{1}$. In what follows, all the dependent and independent variables are converted into non-dimensional form using the first baroclinic dry gravity wave speed $c=N H / \pi \approx 50 \mathrm{~m} / \mathrm{s}$, the equatorial deformation length $L=\sqrt{c / \beta} \approx 1500 \mathrm{~km}$, the time scale $T=L / c \approx 8$ hours, the temperature scale $\bar{\alpha}=H N^{2} \theta_{0} / \pi g=(H / \pi) \bar{\theta}_{z} \approx 15 \mathrm{~K}$, and the pressure scale $c^{2}$. 
When the primitive equations are Galerkin projected into the barotropic and first baroclinic modes according to the ansatz above, we obtain the following nonlinearly coupled shallow-water like systems, for the barotropic and first baroclinic modes, respectively, which were derived and used in [23] and motivated by [27]. We have

$$
\begin{aligned}
& \frac{\partial \overline{\mathbf{v}}}{\partial t}+\overline{\mathbf{v}} \cdot \nabla \overline{\mathbf{v}}+\mathbf{v}_{1} \cdot \nabla \mathbf{v}_{1}+\left(\nabla \cdot \mathbf{v}_{1}\right) \mathbf{v}_{1}+y \overline{\mathbf{v}}^{\perp}=-\nabla \bar{p} \\
& \nabla \cdot \overline{\mathbf{v}}=0 \\
& \frac{\partial \mathbf{v}_{1}}{\partial t}+\overline{\mathbf{v}} \cdot \nabla \mathbf{v}_{1}+\mathbf{v}_{1} \cdot \nabla \overline{\mathbf{v}}+y \mathbf{v}_{1}^{\perp}-\nabla \theta_{1}=0 \\
& \frac{\partial \theta_{1}}{\partial t}+\overline{\mathbf{v}} \cdot \nabla \theta_{1}-\nabla \cdot \mathbf{v}_{1}=S_{\theta}
\end{aligned}
$$

in the above nondimensional units [23, 28, 29, 24, 25, 26]. Here we assumed $S_{\mathbf{V}}=0$ and $S_{\Theta}=S_{\theta} \sqrt{2} \sin (\pi z / H)$. Notice that according to the non-dimensional units introduced above, both the $\beta$ parameter and potential temperature stratification gradient $\bar{\theta}_{z}$ have been normalized to one.

The first two equations in (3.3) are in essence the vertical average of the primitive equation (3.1). This is consistent with the fact that the barotropic mode is independent of height.

The equations in (3.3) have some interesting mathematical properties, among which we have conservation of total (dry) energy. We have

$$
0=\frac{d}{d t} E_{d}(t) \equiv \frac{1}{2} \frac{d}{d t} \iint|\overline{\mathbf{v}}|^{2}+\left|\mathbf{v}_{1}\right|^{2}+\theta_{1}^{2} d x d y
$$

under the appropriate boundary conditions: periodic in $x$ and vanishing meridional velocity at the North and South boundaries [23, 28, 29, 24, 25, 26]. In fact, it is easy to see that under these boundary conditions, the individual, barotropic $\left(\frac{1}{2} \iint|\overline{\mathbf{v}}|^{2} d x d y\right)$ and baroclinic $\left(\frac{1}{2} \iint\left|\mathbf{v}_{1}\right|^{2}+\theta_{1}^{2} d x d y\right)$, energies are conserved independently when the barotropic-baroclinic cross-interaction terms in (3.3) and the advection by the barotropic wind are ignored [25]. This is used as a design principle in [25] to build a numerical scheme for the barotropic-baroclinic coupled system in (3.3). These equations form an interesting laboratory to study the interactions between tropical waves and the extra-tropics. Some work in this direction will be reviewed below and in Section 6 . But many questions of mathematical and scientific interest remain, namely, existence, uniqueness, and regularity of solutions, nonlinear and linear stability of simple and asymptotic solutions such as those discussed below.

3.1.2. Linear wave solutions and meridional structure When the barotropic-baroclinic cross-interaction terms are ignored, the equations in (3.3) split naturally into a nonlinear rotating 2D incompressible Euler equations for the barotropic flow,

$$
\begin{aligned}
& \frac{\partial \overline{\mathbf{v}}}{\partial t}+\overline{\mathbf{v}} \cdot \nabla \overline{\mathbf{v}}+y \overline{\mathbf{v}}^{\perp}=-\nabla \bar{p}, \\
& \nabla \overline{\mathbf{v}}=0,
\end{aligned}
$$


and a rotating linear shallow-water system for the baroclinic mode

$$
\begin{aligned}
& \frac{\partial \mathbf{v}_{1}}{\partial t}+y \mathbf{v}_{1}^{\perp}-\nabla \theta_{1}=0 \\
& \frac{\partial \theta_{1}}{\partial t}-\nabla \cdot \mathbf{v}_{1}=S_{\theta} .
\end{aligned}
$$

Next, we describe simple solutions to each of these systems.

Because of the incompressibility constraint, the barotropic equations can be written in vorticity-stream function form. Let $\bar{\xi}=-\bar{u}_{y}+\bar{v}_{x}$ be the vertical vorticity of the barotropic flow and $\bar{\psi}$ be the associated stream function. We have

$$
\begin{aligned}
& \frac{\partial \bar{\xi}}{\partial t}+J(\bar{\psi}, \xi)+\beta \psi_{x}=0, \\
& \Delta \bar{\psi} \equiv \bar{\psi}_{x x}+\bar{\psi}_{y y}=0 \\
& \bar{u}=-\bar{\psi}_{y}, \bar{v}=\bar{\psi}_{x} \\
& J(f, g)=f_{x} g_{y}-f_{y} g_{x} .
\end{aligned}
$$

Just like 2D incompressible Euler equations, these equations have many conserved quantities, including energy, enstrophy, and potential vorticity $\bar{q}=\bar{\xi}+\beta y$. $\ddagger$ Moreover, because of the variation of the Coriolis force, the $\beta y$ term, these equations possess wavelike solutions known as Rossby waves, which in effect form the weather systems that we see on forecast maps every day [22].

With the ansatz $\bar{\psi}=\hat{\psi} e^{i(k x+l y-\bar{\omega} t)}$, where $k, l$ are respectively the zonal and meridional wavenumbers, $\bar{\omega}$ is the frequency or phase, and $i^{2}=-1$, we have the dispersion relation

$$
\bar{\omega}=-\frac{k}{k^{2}+l^{2}} .
$$

We note that, although this is a linear wave ansatz, it is actually a solution to the fully non-linear equations because the corresponding Jacobian, $J(\bar{\psi}, \Delta \bar{\psi})$, is zero. We also note that, unlike the baroclinic waves discussed next, these barotropic Rossby waves travel Northward and Southward toward the midlatitudes. This interesting feature makes these waves an interesting engine for energy exchange between the tropics and the extra-tropics. Thus, their non-linear interactions with the baroclinic waves is of particular interest.

On the other hand, the baroclinic system in (3.6) has solutions that are trapped near the equator and do not propagate northward or southward. This is the theory of equatorially trapped waves, which can be found in almost any good text on large scale geophysical fluid dynamics [30, 31, 22, 32, etc], and which first appeared in a paper by Matsuno [33]. Next, the simplest of these waves (the Kelvin wave) is described, and then the system is considered in more generality.

When the forcing is ignored $\left(S_{\theta}=0\right)$, the simplest solution for the linear equatorial shallow-water equations (3.6) is obtained by setting $v=0$ (and the subscript 1 being ignored for simplicity). The meridional momentum equation then becomes a geostrophic

$\ddagger$ Recall that $\beta=1$ in the non-dimensional units. 
balance formula between $u$ and $\theta$ in the meridional direction: $y u=\theta_{y}$. Plugging this into the $u$ and $\theta$ equations and then combining the two leads to the wave equation $u_{t t}-u_{x x}=0$, whose solutions of the form $u(x, y, t)=\phi_{ \pm}(x \pm t, y)$ satisfy

$$
y \phi_{t}-\phi_{y x}=0 .
$$

This yields the two solutions $u_{+}(x, y, t)=\phi_{+}(x+t, 0) \exp \left(y^{2} / 2\right)$ and $u_{-}(x, y, t)=\phi_{-}(x-$ $t, 0) \exp \left(-y^{2} / 2\right)$, corresponding to westward and eastward moving waves, respectively, along the equator. It turns out that only the eastward wave is retained as a physically relevant solution; this is not only because it has finite energy and rapidly vanishes as $y \longrightarrow \pm \infty$, but also because it is one of the most observed waves in the tropics $[22,21]$. It is known as a Kelvin wave because it is related to oceanic Kelvin waves that are observed to propagate along the coasts and it has the general waveform $u(x, y, t)=-\theta(x, y, t)=\phi(x-t) \exp \left(-y^{2} / 2\right)$.

Besides the Kelvin wave, there are other equatorially trapped waves that can be seen by considering solutions to (3.6) in more generality. Assuming a wave-like ansatz $(u, v, \theta)^{T}=(\hat{u}, \hat{v}, \hat{\theta})^{T} \exp [i(k x-\omega t)]$ for (3.6) and substituting $\hat{u}$ and $\hat{\theta}$ in the $v$-equation yields a single equation for $\hat{v}$ :

$$
\frac{\partial^{2} \hat{v}}{\partial y^{2}}+\left[\left(\omega^{2}-k^{2}-\frac{k}{\omega}\right)-y^{2}\right] \hat{v}=0,
$$

which is in fact the harmonic-oscillator of quantum mechanics. (See [22] for a treatment using symmetric hyperbolic systems and the raising and lowering operators of quantum mechanics directly for the equatorial shallow water equations without forming the reduced higher order equation in (3.8).) We have the cubic equation,

$$
\omega^{2}-\frac{k}{\omega}-k^{2}=2 n+1 ; \quad n=0,1,2, \cdots,
$$

for the dispersion relation $\omega=\omega(k)$. The Kelvin wave corresponds to $n=-1$ and is not included here; and the special case $n=0$ is considered separately below. For each of $n=1,2, \cdots$, this equation has three solutions: two fast-moving waves, one eastward and one westward, known as inertia-gravity waves; and a westward-moving slow wave, known as a Rossby wave. It is easy to see this if we pass to the asymptotic limit. When $\omega / k>>1$ we obtain a quadratic equation whose solutions, $\omega_{ \pm} \approx \pm\left(k^{2}+2 n+1\right)^{1 / 2}$, are reminiscent of the eastward and westward gravity waves of the classical shallow water equations. On the other hand, when $\omega / k<<1$, we get a single solution $\omega \approx-k /\left(k^{2}+2 n+1\right)$ which is much like the barotropic Rossby wave dispersion relation above if we set $l^{2}=2 n+1$. The integral number $n$, known as the meridional index, is thus closely related to meridional wavenumber.

The special case $n=0$ has only two solutions, the third one being unphysical [32]. One solution corresponds to an eastward moving gravity-like wave and the other one moves westward like a Rossby wave, and thus they are often referred to as the mixed Rossby-gravity or MRG waves. It is also called the Yanai wave.

The dispersion curves $\omega=\omega(k)$ are plotted in Figure 3 for various values of the meridional index $n$. In this plot, eastward-moving waves are displayed on the positive 
side of the zonal wavenumber axis, and westward-moving waves are displayed on the negative $k$ side. Note in particular that the Yanai or MRG dispersion curves which behave like a gravity wave for large positive $k$ and like a Rossby wave for negative $k$. Note the large scale gap between the Rossby wave dispersion curves and those corresponding to gravity waves. This scale gap is often exploited in asymptotic analysis to filter away the fast moving gravity waves such as in the long-wave approximation equations [30, 31, 22]. The Kelvin and Yanai waves, however, behave very differently: they include both high frequency and low-frequency modes.

To find the structures of the waves, (3.8) must be solved. With the dispersion relation in (3.9), the eigensolutions of (3.8) are given by

$$
\hat{v}(y)=v_{0} H_{n}(y) \exp \left(-y^{2} / 2\right), n=0,1,2, \cdots
$$

where $H_{n}(y), n=0,1,2, \cdots$ are the Hermite polynomials with $H_{0}(y)=1, H_{1}(y)=$ $2 y, H_{2}(y)=4 y^{2}-2, \cdots$. The functions $D_{n}(y)=C_{n} H_{n}(y) \exp \left(-y^{2} / 2\right), n=0,1,2, \cdots$ are the parabolic cylinder functions, which form an orthonormal basis for the $L^{2}$ inner product on $(-\infty,+\infty)$. Here $C_{n}, n=0,1,2, \cdots$ are the normalization constants. Let $L_{ \pm} \equiv \frac{d}{d y} \pm y$ be the raising and lowering operators of quantum mechanics. Then

$$
L_{-} D_{n}(y)=-\sqrt{2(n+1)} D_{n+1}(y) \text { and } L_{+} D_{n}(y)=\sqrt{2 n} D_{n-1}(y) .
$$

If we introduce the Riemann invariants $q=(u-\theta) / \sqrt{2}$ and $r=(u+\theta) / \sqrt{2}$, then the (unforced) equatorial shallow water equations (3.6) yield a symmetric hyperbolic system whose eigenmodes involve the parabolic cylinder functions [22]. In particular, we have

$$
\begin{aligned}
& i(k-\omega) \hat{q}(y)=-\frac{1}{\sqrt{2}} L_{-} \hat{v}(y) \\
& i(k+\omega) \hat{r}(y)=-\frac{1}{\sqrt{2}} L_{+} \hat{v}(y) \\
& i \omega \hat{v}(y)=\frac{1}{\sqrt{2}}\left(L_{+} \hat{q}(y)-L_{-} \hat{r}(y)\right) .
\end{aligned}
$$

With (3.10), we have all the normal modes of the equatorial shallow water equations expanded in terms of the parabolic cylinder functions. These waves vanish exponentially fast away from the equator, and they travel in both directions along the equator - thus the name "equatorially trapped waves." More details on these waves can be found in the literature $[30,31,22,32,21]$ and therefore they are not discussed further here. However they will reappear in the upcoming sections in the form of moisture-coupled or convectively coupled waves. 


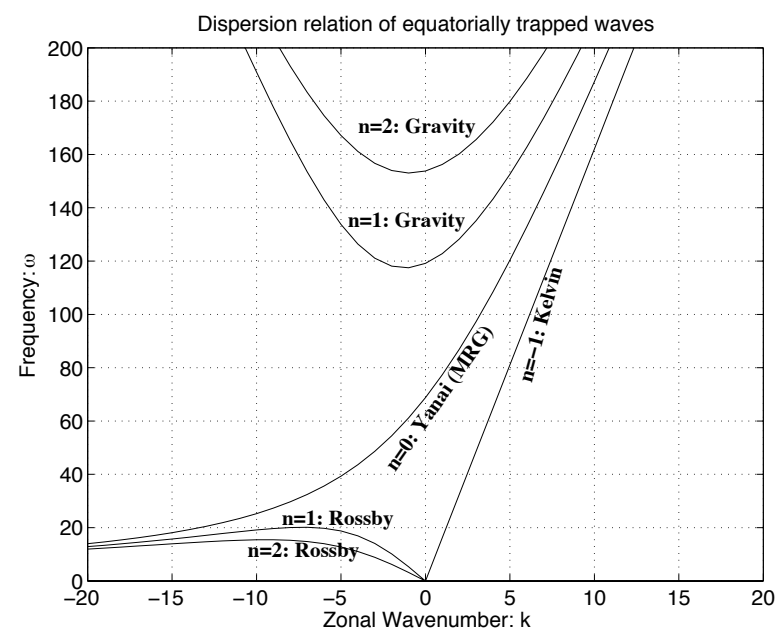

Figure 3. Dispersion relation of equatorially trapped waves. Various dispersion curves corresponding to the first few meridional indices are shown. See text for details. The curves on the right of $k=0$ correspond to eastward moving waves and those on the left correspond to westward waves.

3.1.3. Weakly nonlinear Kelvin waves We consider the nonlinear equatorial shallow water equations,

$$
\begin{aligned}
& \frac{\partial u}{\partial t}+u \frac{\partial u}{\partial x}+v \frac{\partial u}{\partial y}-y v-\frac{\partial \theta}{\partial x}=0 \\
& \frac{\partial v}{\partial t}+u \frac{\partial v}{\partial x}+v \frac{\partial v}{\partial y}+y u-\frac{\partial \theta}{\partial y}=0 \\
& \frac{\partial \theta}{\partial t}+u \frac{\partial \theta}{\partial x}+v \frac{\partial \theta}{\partial y}-(1-\theta)\left(u_{x}+u_{y}\right)=0 .
\end{aligned}
$$

We note the equations in (3.11) are not directly derived from the primitive equations (3.1) as the coupled system in (3.3), but they bear a lot of resemblance to them. In particular, their linearized version is identical to the baroclinic equations in (3.6). Here, we are going to use them as an ad hoc model to gain some insight into the effects of the advection nonlinearities on the Kelvin waves. This was done originally in [34] but most of the details can be found in [22]. We introduce a small parameter $\epsilon$ and assume the ansatz

$$
\left(\begin{array}{l}
u \\
\theta \\
v
\end{array}\right)=\epsilon\left(\begin{array}{c}
\phi(x-t, \tau) \exp \left(-y^{2} / 2\right) \\
-\phi(x-t, \tau) \exp \left(-y^{2} / 2\right) \\
0
\end{array}\right)+\epsilon^{2}\left(\begin{array}{l}
u_{2} \\
\theta_{2} \\
v_{2}
\end{array}\right),
$$

where $\tau=\epsilon t$ is a slow time on which amplitude modulations for the Kelvin waves are allowed, and $\left(u_{2}, \theta_{2}, v_{2}\right)^{T}$ is the second order correction. Here $\phi=\phi(\zeta, \tau)$ with $\xi=x-t$. We are interested in the evolution in this ansatz on the $\tau$ time scale. Therefore, as a solvability condition [34, 22], we require a sublinear growth condition for the high order correction term in terms of the fast time $t:\left(u_{2}, \theta_{2}, v_{2}\right)^{T}=o(t)$ uniformly for $0 \leq t \leq \tau$. Otherwise $\left.\left(u_{2}, \theta_{2}, v_{2}\right)^{T}\right|_{\tau=1}=\left.\left(u_{2}, \theta_{2}, v_{2}\right)^{T}\right|_{t=1 / \epsilon} \geq O(1 / \epsilon)$ and the ansatz (3.12) will break down. 
Plugging in this ansatz into (3.11) and collecting together terms of the same order in $\epsilon$ yields the following system for the correction term,

$$
\begin{aligned}
& \frac{\partial u_{2}}{\partial t}-\frac{\partial \theta_{2}}{\partial x}-y v_{2}=-\phi_{\tau} \exp \left(-y^{2} / 2\right)-\phi \phi_{\zeta} \exp \left(-y^{2}\right) \\
& \frac{\partial v_{2}}{\partial t}-\frac{\partial \theta_{2}}{\partial y}+y u_{2}=0 \\
& \frac{\partial \theta_{2}}{\partial t}-\frac{\partial u_{2}}{\partial x}-\frac{\partial v_{2}}{\partial y}=\phi_{\tau} \exp \left(-y^{2} / 2\right)+2 \phi \phi_{\zeta} \exp \left(-y^{2}\right),
\end{aligned}
$$

from the $O\left(\epsilon^{2}\right)$ terms, while the $O(\epsilon)$ terms vanish automatically. For the sublinear growth condition, the projection of the right hand side onto the Kelvin mode must be zero.

Passing to Riemann invariant variables in the linear shallow water equations (3.6), $(q=u-\theta, r=u+\theta, v)^{T}$, it is easy to see that for the Kelvin wave we have $r=0, v=0$ and $q$ satisfies $\frac{\partial q}{\partial t}+\frac{\partial q}{\partial x}=0$. Thus it is enough to look at the equation satisfied by $q_{2}=u_{2}-\theta_{2}$ from the equations in (3.13) when projected on the meridional structure function $\exp \left(-y^{2} / 2\right)$. If $v_{2}$ does not grow as $\exp \left(y^{2}\right)$ as $y \longrightarrow \infty$, then we get the following equation

$$
\frac{\partial \tilde{q}_{2}}{\partial t}+\frac{\partial \tilde{q}_{2}}{\partial x}=-\lim _{L \longrightarrow \infty} \frac{1}{L} \int_{-L}^{+L}\left(2 \phi_{\tau} e^{-y^{2}}+3 \phi \phi_{\zeta} e^{-3 y^{2} / 2}\right) d y
$$

where $\tilde{q}_{2}=\lim _{L \longrightarrow \infty} \frac{1}{L} \int_{-L}^{+L} e^{-y^{2} / 2} q_{2} d y$.

Therefore, the sublinear growth condition leads us to the famous Burgers equation $\phi_{\tau}+c\left(\phi^{2}\right)_{\zeta}=0$ for the amplitude function $\phi$ of the Kelvin wave, where $c=$ $(3 / 2) \int_{-\infty}^{+\infty} \exp \left(-3 y^{2} / 2\right) d y / 2 \int_{-\infty}^{+\infty} \exp \left(-y^{2}\right) d y$. See [34] for exact solutions and simple numerical experiments illustrating some remarkable effects of topography and nonlinear Kelvin waves at large scales which can prevent the breaking of waves through subtle large scale dispersion.

3.1.4. Nonlinear interaction of first and second baroclinic modes Now, we consider the ansatz,

$$
\begin{aligned}
& \mathbf{V}=\overline{\mathbf{v}}+\sqrt{2} \cos (z) \mathbf{v}_{1}+\sqrt{2} \cos (2 z) \mathbf{v}_{2}, \\
& W=\sqrt{2} \sin (z) w_{1}+\frac{\sqrt{2}}{2} \sin (2 z) w_{2}=-\sqrt{2} \sin (z) \nabla \cdot \mathbf{v}_{1}-\frac{\sqrt{2}}{2} \sin (2 z) \nabla \cdot \mathbf{v}_{2} \\
& \Phi=\bar{p}+\sqrt{2} \cos (z) p_{1}+\sqrt{2} \cos (2 z) p_{2}, \\
& \Theta=\theta_{0}+\frac{d \bar{\theta}}{d z} z+\sqrt{2} \sin (z) \theta_{1}+2 \sqrt{2} \sin (2 z) \theta_{2}, 0 \leq z \leq \pi,
\end{aligned}
$$

of projecting the primitive equations (3.1) into the first three modes of vertical structure, the barotropic mode and the first two baroclinic modes. We obtain three systems of PDEs $[35,36]$ : the usual incompressible rotating 2D Euler equations for the barotropic mode,

$$
\begin{aligned}
& \frac{\bar{D} \overline{\mathbf{v}}}{D t}+y \overline{\mathbf{v}}^{\perp}+\nabla \bar{p}=-\left(\nabla \cdot\left(\mathbf{v}_{1} \otimes \mathbf{v}_{1}\right)+\nabla \cdot\left(\mathbf{v}_{2} \otimes \mathbf{v}_{2}\right)\right) \\
& \nabla \cdot \overline{\mathbf{v}}=0
\end{aligned}
$$


and two equatorial shallow water systems for the first two baroclinic modes,

$$
\begin{aligned}
& \frac{\bar{D} \mathbf{v}_{1}}{D t}+\mathbf{v}_{1} \cdot \nabla \overline{\mathbf{v}}-\nabla \theta_{1}+y \mathbf{v}_{1}^{\perp}=S_{1}^{\mathbf{v}}-\frac{\sqrt{2}}{2}\left(\mathbf{v}_{1} \cdot \nabla \mathbf{v}_{2}+\mathbf{v}_{2} \cdot \nabla \mathbf{v}_{1}+\frac{1}{2} \mathbf{v}_{1} \nabla \cdot \mathbf{v}_{2}+2 \mathbf{v}_{2} \nabla \cdot \mathbf{v}_{1}\right) \\
& \frac{\bar{D} \theta_{1}}{D t}-\nabla \cdot \mathbf{v}_{1}=S_{1}^{\theta}-\frac{\sqrt{2}}{2}\left(2 \mathbf{v}_{1} \cdot \nabla \theta_{2}-\mathbf{v}_{2} \cdot \nabla \theta_{1}+4 \theta_{2} \nabla \cdot \mathbf{v}_{1}-\frac{1}{2} \theta_{1} \nabla \cdot \mathbf{v}_{2}\right) \\
& \text { and }
\end{aligned}
$$

$$
\begin{aligned}
& \frac{\bar{D} \mathbf{v}_{2}}{D t}+\mathbf{v}_{2} \cdot \nabla \overline{\mathbf{v}}-\nabla \theta_{2}+y \mathbf{v}_{2}^{\perp}=S_{2}^{\mathbf{v}}-\frac{\sqrt{2}}{2}\left(\mathbf{v}_{1} \cdot \nabla \mathbf{v}_{1}-\mathbf{v}_{1} \nabla \cdot \mathbf{v}_{1}\right) \\
& \frac{\bar{D} \theta_{2}}{D t}-\frac{1}{4} \nabla \cdot \mathbf{v}_{2}=S_{2}^{\theta}-\frac{\sqrt{2}}{4}\left(\mathbf{v}_{1} \cdot \nabla \theta_{1}-\theta_{1} \nabla \cdot \mathbf{v}_{1}\right) .
\end{aligned}
$$

Here $\frac{\bar{D}}{D t} \equiv \frac{\partial}{\partial t}+\overline{\mathbf{v}} \cdot \nabla$ is the barotropic transport operator and the hydrostatic and continuity relationships, $\theta_{j}=-p_{j}, w_{j}=-\frac{1}{j} \nabla \cdot \mathbf{v}_{j}, j=1,2$, were used.

When the barotropic mode variations as well as the effects of rotation and meridional dependence are ignored all together, we obtain a two-mode shallow water system (as opposed to the two-layer shallow water system, which is extensively studied in the applied mathematics community). This is for a one-dimensional flow above the equator that governs the dynamics of nonlinear hydrostatic gravity waves [35]. In [35], this system is written in the advective form

$$
\frac{\partial \mathbf{u}}{\partial t}+\mathbf{A}(\mathbf{u}) \frac{\partial \mathbf{u}}{\partial x}=\mathbf{S}
$$

where

$$
\mathbf{u}=\left(\begin{array}{c}
u_{1} \\
\theta_{1} \\
u_{2} \\
\theta_{2}
\end{array}\right), \mathbf{A}=\left[\begin{array}{cccc}
\bar{u}+\frac{\sqrt{3}}{2} u_{2} & -1 & \frac{1}{2} u_{1} & 0 \\
1+2 \sqrt{2} \theta_{2} & \bar{u}-\frac{1}{\sqrt{2}} u_{2} & -\frac{1}{2 \sqrt{2}} \theta_{1} & \sqrt{2} u_{1} \\
0 & \bar{u} & 0 & -1 \\
-\frac{1}{2 \sqrt{2}} \theta_{1} & \frac{1}{2 \sqrt{2}} u_{1} & -\frac{1}{4} & \bar{u}
\end{array}\right]
$$

$\bar{u}$ is a constant barotropic wind and $\mathbf{S}$ is the corresponding source term vector.

The system in (3.18) has many important mathematical properties: i) it conserves total energy, $\mathcal{E}=\frac{1}{2}\left(u_{1}^{2}+u_{2}^{2}+\theta_{1}^{2}+4 \theta_{2}^{2}\right)$, ii) it is nonconservative, iii) it is not always hyperbolic, and iv) its eigenmodes are neither genuinely nonlinear nor linearly degenerate [35]. Nevertheless, a robust numerical method was designed in [35] for this system. The strategy is to split the advective system into conservative and nonconservative parts as

$$
\mathbf{A}(\mathbf{u}) \frac{\partial \mathbf{u}}{\partial x}=\frac{\partial \mathbf{F}(\mathbf{u})}{\partial x}+\mathbf{A}_{n c}(\mathbf{u}) \frac{\partial \mathbf{u}}{\partial x}
$$

using the classical operator time splitting methodology of Strang [37]. While there are many other ways of splitting this system, the strategy adopted in [35] was to choose $A_{n c}$ so that all its eigenvalues are zero, i.e, a nilpotent matrix, so that simple centered differences could be used for the corresponding $x$-derivatives without the risk of numerical instabilities. For the conservative part, Stechmann et al. [35] used a non-oscillatory central scheme [38, 39].

Although the eigenvalues of the matrix $\mathbf{A}$ in (3.18) are not accessible analytically, they are easily computed numerically. It is found in [35] that the system (3.18) typically 

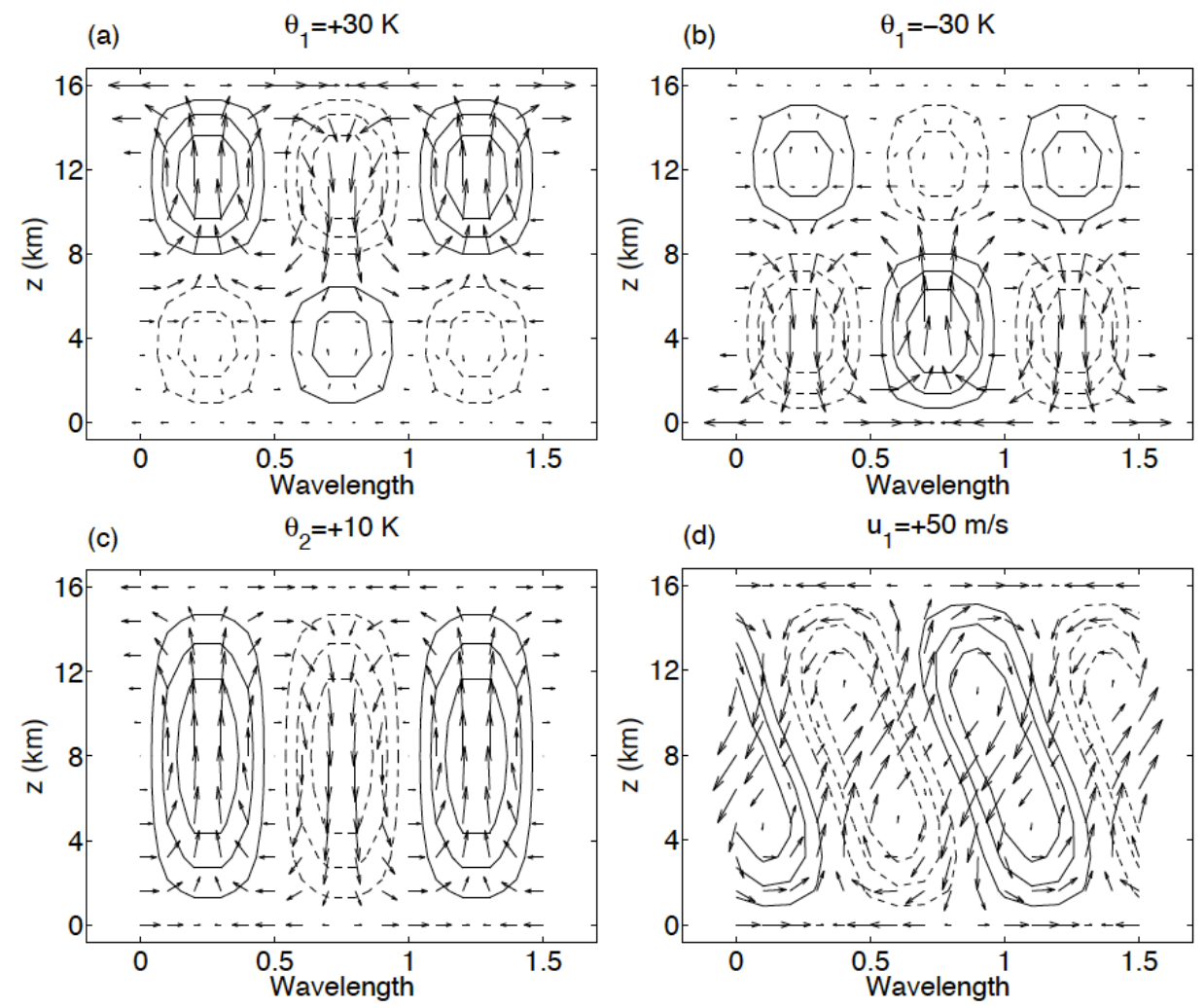

Figure 4. Unstable waves for various non-hyperbolic background states. In each case, only one of $u_{1}, u_{2}, \theta_{1}, \theta_{2}$ is nonzero: (a) $\theta_{1}=+30 \mathrm{~K}$, (b) $\theta_{1}=\mid 30 \mathrm{~K}$, (c) $\theta_{2}=+10 \mathrm{~K}$, and (d) $u_{1}=+50 \mathrm{~m} / \mathrm{s}$. Solid contours are positive anomalies of potential temperature, and dashed contours are negative anomalies.

loses its hyperbolicity for large absolute values of $u_{1}$ and $\theta_{1}$ and for large positive values of $\theta_{2}$. Note that a large negative $\theta_{2}$ perturbation does not affect hyperbolicity because it enhances the stable stratification; i.e., it injects cold air in the lower troposphere and warm air aloft. On the other hand, a large positive $\theta_{2}$ creates an unstable stratification by putting cold air on top of warm air. Four typical eigenmodes that arise when the matrix loses its hyperbolicity are illustrated in Figure 4. The thermodynamic cases (a),(b),(c) in Figure 4 have the important common physical feature of having upward flow overlying warm temperature anomalies and downward flow on top of cold anomalies. Intuitively, this feature has the potential of stabilizing the background flow by bringing cold air down and pushing warm air up. Notice also that the panels in (a) and (b) have stronger anomalies in the upper and lower troposphere, respectively. This is physically consistent with the fact that the $\sin (z)$ profile of $\theta_{1}$ is (convectively) stable in the lower troposphere and unstable in the upper troposphere when $\theta_{1}>0$ (and vice-versa if $\left.\theta_{1}<0\right)$. These peculiar flow structures are very different from the free (classical) first 

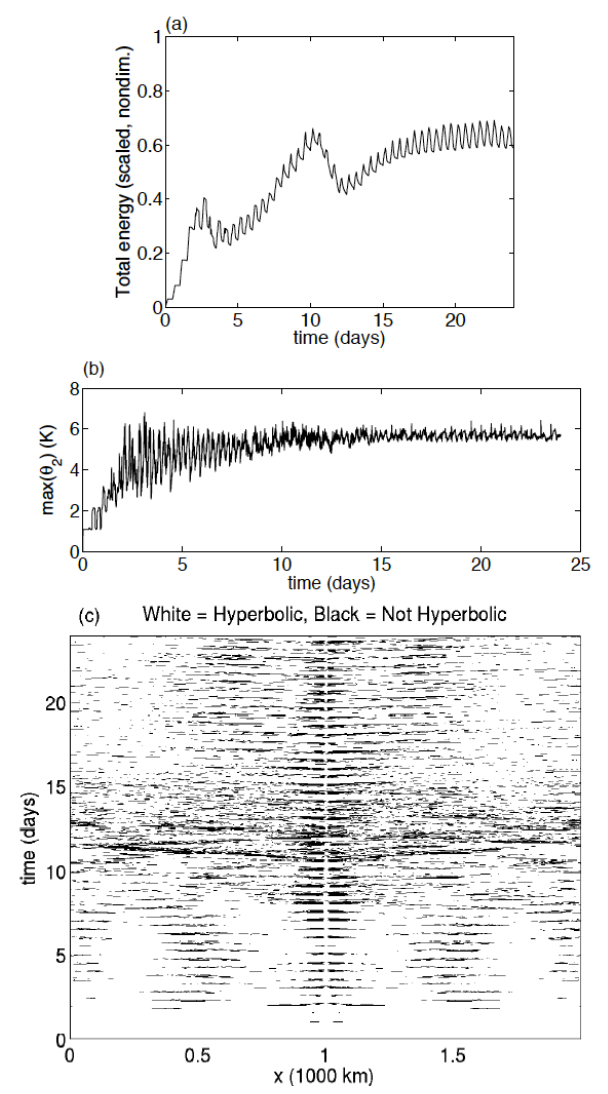

Figure 5. Results for a simulation with the imposed source terms (23) that force the system into non-hyperbolic states. (a) Time evolution of the total energy. (b) Time evolution of the domain-maximum of $\theta_{2}$, which never exceeds $\approx 6 \mathrm{~K}$. (c) Points (x, t) where hyperbolicity was lost. White squares represent hyperbolic points and black squares represent non-hyperbolic points.

and second baroclinic gravity modes, which correspond to the homogeneous background state of $u_{1}=u_{2}=\theta_{1}=\theta_{2}=0$, and which have flow patterns that are aligned with the temperature contours (see Figure 4 of [35]).

For the particular case of $u_{1}=u_{2}=\theta_{1}=0$, it is shown in [35] that the matrix $\mathbf{A}$ becomes non-hyperbolic when $\theta_{2}>2^{-3 / 2}$ ( 5 Kelvin). This is a relatively large value for tropical flows, which support only small temperature perturbations on the order of 1-2 K. However, to test the robustness of the numerical method, the system (3.18) was forced artificially by an imposed, unrealistically large heat source in [35]. A periodic pulse with a Gaussian spatial structure $S_{\theta_{1}}=2 S_{\theta_{2}}=a H(t) \exp \left(-x^{2} / 2 \sigma^{2}\right), S_{u_{1}}=S_{u_{2}}=0$ with $\sigma=20 \mathrm{~km}$ and $a=200 \mathrm{~K} /$ day, centered in a $2000 \mathrm{~km}$ periodic domain is 
used. While the system is strongly pushed towards the non-hyperbolic region, the numerical solution does not blow up; rather, it stabilizes and weakly oscillates between hyperbolic and non-hyperbolic states as shown in Figure 5 (see top and bottom panels, respectively). Also shown in Figure 5 is the time series of the $\theta_{2}$ anomaly. Interestingly, this variables saturates somewhere between 5 and $6 \mathrm{~K}$, i.e, close to the value of $5 \mathrm{~K}$ where the system is found to lose hyperbolicity when the other variables are all zero. This poses an interesting paradigm for the plausibility of a close relationship between the mathematical notion of hyperbolicity and the notion of hydrodynamic stability.

The interested reader is referred to the original article [35] for the construction of simple nonlinear wave solutions using the method of characteristics. Both breaking and non-breaking waves are exemplified to demonstrate the linear degeneracy and the genuine nonlinearity of (3.18). The behavior of these waves in an imposed background shear were considered in [40] and their possible impact on the development and initiation of deep convection is demonstrated. The extension of the Galerkin projection to three and four baroclinic modes of vertical structure and the impact of including more vertical modes, on the dynamical behavior of the gravity waves, in terms of favoring or not deep convection, are also discussed in [40].

\subsection{Moist dynamics}

A major complexity of tropical atmospheric circulation is associated with clouds and precipitation that involve latent heat release due to phase change of water and important changes in the radiative budget. These convective and radiative processes interact and force the equatorially trapped waves discussed above and give rise to large scale convectively coupled waves, the MJO, and climatological patterns such as the Hadley and Walker circulations reviewed in the previous section. To illustrate some of the complex dynamics of the large-scale tropical circulation and convectively coupled waves, we first derive an equation for the dynamics of the large scale moisture.

3.2.1. The large-scale moisture equation We consider the bulk cloud microphysics equations

$$
\begin{aligned}
& \frac{D \Theta}{D t}+W \frac{\partial \bar{\theta}}{\partial z}=\frac{L_{v}}{C_{p}}\left(C_{d}-E_{r}-E_{c}\right)+Q_{R} \\
& \frac{D q_{v}}{D t}=-C_{d}+E_{c}+E_{r} \\
& \frac{D q_{c}}{D t}=C_{d}-E_{c}-C_{r} \\
& \frac{D q_{r}}{D t}+\frac{\partial\left(v_{T} q_{r}\right)}{\partial z}=C_{r}-E_{r},
\end{aligned}
$$

where $q_{v}, q_{c}, q_{r}$ are the mixing ratios of water vapor, cloud water, and rain, respectively

and $\frac{D}{D t}=\frac{\partial}{\partial t}+\mathbf{V} \cdot \nabla+W \frac{\partial}{\partial z}$ is the transport derivative with respect to the $3 \mathrm{D}$ flow. Other species involving the ice phase of water are ignored here for simplicity in exposition. 
However, in operational weather and climate simulations, ice is included systematically in terms of cloud ice, snow, hail, and graupel. Ice affects not only the structure of clouds themselves but also their impact on the radiative budget: water vapor and clouds are major players in the greenhouse effect of Earth's atmosphere. However, the cloud radiative feedback and other greenhouse effects are ignored in the hierarchy of simple models presented in this review.

In (3.19), $C_{d}, E_{r}, E_{c}, C_{r}$ are the rates of condensation of vapor to cloud water, evaporation of rain, evaporation of cloud water, and conversion of cloud water to rain, respectively. Also, $L_{v}$ and $C_{p}$ are the latent heat of vapor and heat capacity of (dry) air at constant pressure, respectively, and $v_{T}$ is the (bulk) terminal velocity of rain drops while $Q_{R}$ is the longwave radiative cooling. In cloud resolving modelling simulations, the rates $C_{d}, E_{r}, E_{c}, C_{r}$ are represented or parameterized according to various recipes based on empirical formulas and/or theoretical models based on detailed cloud microphysics (see [41, 42] and references therein). The parameterization of cloud condensation and evaporation rates, in particular, are based on the assumption of thermodynamic equilibrium of water: if $q_{v}$ is pushed above its saturation value $q_{v, s}$, then it adjusts instantaneously back to the saturation value, and $q_{v, s}$ is a function of temperature according to the Clausius-Clapeyron equation [30].

The equations (3.19) have two important conserved quantities. Namely, both moist static energy $m=\frac{d \bar{\theta}}{d z} z+\Theta+\frac{L_{v}}{C_{p}} q_{v}$ ( since $\frac{D z}{D t}=W$ ) and total water content $q^{\text {tot }}=q_{v}+q_{c}+q_{r}$ are simply advected by the flow in the absence of radiation $\left(Q_{R}=0\right)$ and of precipitation $\left(v_{t}=0\right)$, respectively; i.e., $D m / D t=0$ and $D q^{t o t} / D t=0$.

Let $q_{l}=q_{c}+q_{r}$ be the liquid water content and $\langle$.$\rangle be the vertical average operator$ over the tropospheric column. Then, under the assumptions of scale separation between clouds and rain formation processes that occur on very fast time scales (minutes to hours) and large scale water vapor and temperature variability, the liquid water content adjusts quickly to its equilibrium value, which is uniquely determined by the large scale values of $q_{v}$ and $T$. Thus, we have $[27,24,43,44]$

$$
\begin{aligned}
& \left\langle C_{d}-E_{c}-E_{r}\right\rangle=\left.v_{t} q_{r}\right|_{z=0} \equiv P \\
& \left\langle\frac{D q_{v}}{D t}\right\rangle=-P
\end{aligned}
$$

where $P$ is the amount of precipitation that reaches the ground and where turbulent fluxes are ignored. In addition, we assume that the large scale moisture decomposes into a background moisture stratification plus some perturbation as $q_{v}=Q(z)+q^{\prime}$, and we set $q=\left\langle q_{v}^{\prime}\right\rangle$. The large scale moisture equation then becomes [24, 44]

$$
\frac{\partial q}{\partial t}+\left\langle\nabla \cdot\left(\mathbf{V} q_{v}^{\prime}\right)\right\rangle+\left\langle W \frac{d Q(z)}{d z}\right\rangle=E_{0}-P
$$

where $E_{0}=\left.\left(w q_{v}^{\prime}\right)\right|_{z=0}$ is the flux of vapor from the surface, which can be thought of as the evaporation from the sea surface, and the identity $\mathbf{V} \cdot \nabla q_{v}^{\prime}+W \frac{\partial q_{v}^{\prime}}{\partial z}=\nabla \cdot\left(\mathbf{V} q_{v}^{\prime}\right)+\frac{\partial}{\partial z}\left(W q_{v}^{\prime}\right)$ is used. The above reasoning is an example of closure modelling which is ubiquitous in GCMs and in climate science. 
3.2.2. Simple one-heating mode model With the Galerkin truncation in 3.1 .1 for reducing the primitive equations to the barotropic and first baroclinic modes, the moisture equation becomes

$$
\frac{\partial q}{\partial t}+\overline{\mathbf{v}} \cdot \nabla q+\tilde{Q} \nabla \mathbf{v}_{1}=E_{0}-P
$$

Here, the evaporation and precipitation rates are parameterized according to $[27,24$, 43, 26]:

$$
E_{0}=d\left(q_{v, s}\left(T_{s}\right)-q\right), \quad P=\frac{1}{\tau_{c}}(q-\tilde{q}(\theta))^{+},
$$

where $T_{s}$ is the surface temperature and $\tilde{q}(\theta)$ is some measure of the level of saturation inside the large scale grid box, a function of temperature, typically $\tilde{q}(\theta)<q_{v, s}(\theta)$. In $[24,43,26]$, the formula $\tilde{q}=\hat{q}+\alpha \theta$ is used where $\hat{q}>0$ and $0 \leq \alpha \leq 1$ are prescribed parameters. When $\alpha=0$ the classical Betts-Miller parameterization [45] is recovered and when $\alpha=1, \hat{q}=0$ the parameterization of [27] is recovered. Here $\tau_{c}$ and $d$ are respectively convective and surface evaporation time scales; typically $\tau_{c}<<d$. Below we will be focussing on the limit $\tau_{c} \longrightarrow 0$. The parameter $\tilde{Q}$ depends of the moisture background $Q(z)$ and the vertical profile of the first baroclinic flow. When $q$ assumes the non-dimensional units of temperature, introduced above, values $0.8 \leq \tilde{Q} \leq 0.9$ are representative of the tropical sounding [46, 24].

The first baroclinic equations in (3.3) and the moisture equation in (3.21) form the minimal model parameterization of tropical convection. It amounts to assuming a single cloud type consisting of deep convective towers that heat the first baroclinic mode without any direct impact on the higher modes [27]. A more realistic parameterization that carries three cloud types and two baroclinic modes will be discussed below in section 5.1. Under the constraint of conservation of moist static energy, we set $S_{\theta}=P+Q_{R}$. In this simple setting, the radiative cooling $Q_{R}$ assumes a simple Newtonian relaxation of temperature towards a prescribed equilibrium value [24]. Next, we give a few important conservation properties for this moisture-coupled system.

The equivalent potential temperature $\theta_{e}=\theta+q$ (in the non-dimensional units) is conserved

$$
\frac{\bar{D} \theta_{e}}{D t}=(1-\tilde{Q}) \nabla \cdot \mathbf{v}_{1}
$$

when both the surface evaporation $E_{0}$ and longwave radiation $Q_{R}$ are ignored. Similarly, the quantity $Z=q+\tilde{Q} \theta$ decreases along particle paths,

$$
\frac{\bar{D} Z}{D t}=-(1-\tilde{Q}) P \leq 0,
$$

provided $\tilde{Q}<1$. Moreover, we define the moist energy as

$$
E_{m}=\frac{1}{2} \int \frac{Z^{2}}{(1-\tilde{Q})(\alpha+\tilde{Q})} d x d y,
$$

and consider the total energy $E=E_{d}+E_{m}$ with $E_{d}$ defined in $(3.4) ; E$ satisfies $[24,43,26]$

$$
E(t)=E(0)-\int_{0}^{t} \int \frac{q-\alpha \theta}{\alpha+\tilde{Q}} P d x d y d t .
$$


Given that $(q-\alpha \theta) P \geq 0$ by the definition of $P$, the last equality expresses a general principle according to which precipitation dissipates energy through a nonlinear mechanism known as cumulus friction.

Now, we assume that the barotropic wind is absent $(\overline{\mathbf{v}}=0)$ and consider the resulting first baroclinic-moisture-coupled system. Let

$$
E_{\text {grad }}(t)=\frac{1}{2} \iint\left|\nabla \mathbf{v}_{1}\right|^{2}+\left|\nabla \theta_{1}\right|^{2}+\frac{|\nabla Z|^{2}}{(1-\tilde{Q})(\alpha+\tilde{Q})} d x d y
$$

be the gradient energy. Then we have [24, 26]

$$
E_{\text {grad }}(t) \leq E_{\text {grad }}(0)+\int_{0}^{t} \int\left(v_{1} \frac{\partial u_{1}}{\partial y}-u_{1} \frac{\partial v_{1}}{\partial y}\right) d x d y
$$

Therefore, by Gronwall's lemma, $E_{\text {grad }}(t)$ remains bounded in finite time uniformly in $\tau_{c}$, although it can grow exponentially fast due to the Coriolis beta effect. Thus, by Sobolev's lemma, discontinuities in $\mathbf{v}_{1}, \theta_{1}, q$ cannot develop from smooth initial data even in the limit when $\tau_{c} \longrightarrow 0$. However, discontinuities in their gradients are allowed.

3.2.3. Limiting dynamic moisture front solutions Let us now look at the behavior of the moisture-coupled system when $\tau_{c} \longrightarrow 0$. For simplicity, we consider the one-dimensional case when the rotation effect and meridional dependences are ignored:

$$
\begin{aligned}
& u_{t}-\theta_{x}=0, \\
& \theta_{t}-u_{x}=P, \\
& q_{t}+\tilde{Q} u_{x}=-P=-\frac{1}{\tau_{c}}(q-\hat{q}-\alpha \theta)^{+} .
\end{aligned}
$$

In (3.25) the subscript 1 is dropped for simplicity in exposition. We now divide the domain into moist and dry regions. Dry regions are characterized by zero precipitation, $P=0$, and moist regions satisfy $P>0$ and have positive moisture convergence, $\tilde{Q} u_{x}<0$. Formally, in the limit $\tau_{c} \longrightarrow 0$, we have the characterization

$$
\begin{aligned}
& \text { Dry region: } \Longleftrightarrow q<\tilde{q}(\theta) \text { or } q=\tilde{q}(\theta) \text { and } \frac{\partial q}{\partial t}<0 \\
& \text { Moist region: } \Longleftrightarrow q=\tilde{q}(\theta) \text { and } \frac{\partial q}{\partial t}>0 .
\end{aligned}
$$

The condition $q=\tilde{q}$ in moist regions and dry regions that have a moisture sink are consistent with the fact from cloud microphysics that condensation and evaporation rates tend to keep the water vapor at its saturation value $q_{v, s}$ when there is contact with liquid water. It is thus sensible to view $\tilde{q}(\theta)$ as the equivalent of the ClausiusClapeyron equation in this simple setting. The conditions in (3.26) define a hyperbolic free boundary problem for the moisture zones.

Within the dry regions, the moisture decouples completely from the dry dynamic variables $u, \theta$ and the associated equations admit gravity waves with a dispersion-less dry gravity-wave phase-speed $c_{d}=1\left(50 \mathrm{~m} \mathrm{~s}^{-1}\right)$. In the moist regions, on the other hand, $q=\hat{q}+\alpha \theta$ and the equation for $q$ and $\theta$ could be combined to eliminate $P$. This results in a shallow water system with a reduced/moist gravity wave speed $c_{m}=\sqrt{\frac{1-\tilde{Q}}{1+\alpha}}$. 
This is consistent with the now universal statement that convectively coupled equatorial waves have a reduced phase speed when compared to their dry analogs [47, 48, 49, 50]; in fact, the phase speed of observed convectively coupled Kelvin waves (CCKWs) is 15 $\mathrm{m} \mathrm{s}^{-1}$ while the dry first baroclinic Kelvin waves (which share similar zonal structure and vertical wavenumber) move at $50 \mathrm{~m} \mathrm{~s}^{-1}$. This is realized by the present model if we set $\tilde{Q}=0.9$ and $\alpha=0$ so that $c_{m}=\sqrt{0.1} \times 50 \mathrm{~m} \mathrm{~s}^{-1} \approx 15 \mathrm{~m} \mathrm{~s}^{-1}$. The physical mechanisms behind this reduction of phase speed are much more complicated than in this simple model. However, this main idea is utilized in [51] to suggest a dependence of the phase-speed of CCWK on the width of the intertropical convergence zone (ITCZ).

We are now ready to formulate global simple exact solutions for the coupled system (3.25) in the form of thin propagating fronts that separate moist and dry regions (3.26), in the limit $\tau_{c} \longrightarrow 0$. We consider the governing equations for the derivatives $u_{x}, \theta_{x}, q_{x}$ that are obtained by differentiating (3.25), and we write the Rankine-Hugoniot jump conditions for a uniform solution within the dry and moist regions on either side of a thin interface:

$$
\begin{aligned}
& s\left[u_{x}\right]=-\left[\theta_{x}\right] \\
& s\left[\theta_{x}\right]=-\left[u_{x}\right]+[P] \\
& s\left[q_{x}\right]=\tilde{Q}\left[u_{x}\right]-[P],
\end{aligned}
$$

where $[f] \equiv f^{+}-f^{-}=f\left(x_{0}^{+}\right)-f\left(x_{0}^{-}\right)$, and $x=x_{0}$ is the location of the interface at time $t$, and we assume $x>x_{0}$ is moist and $x<x_{0}$ is dry. This leads to a family of propagating moisture fronts with speeds given by

$$
s= \pm\left(\frac{c_{m}^{2} w^{+}-w^{-}}{w^{+}-w^{-}}\right)^{1 / 2}
$$

where $w$ is a rescaled vertical velocity so that $w=-u_{x}$. In order to have a real-valued front speed $s$, one of the conditions $w^{-} \leq c_{m}^{2} w^{+}$or $w^{-}>w^{+}>0$ must be satisfied. Therefore, only three types of moisture fronts are admissible [24]:

Drying fronts with moderate speeds: $c_{m} \leq s<c_{d}$,

Slow moistening fronts with $-c_{m} \leq s<0$,

Fast moistening fronts with $s<-c_{d}$.

An algorithm for the systematic construction of such moisture front solutions can be found in $[43,26]$. It is interesting to note that according to the Lax stability criterion for the stability of moving shocks, only the drying fronts are stable because they satisfy $c_{m}<s<c_{d}$. However, at finite relaxation time, $\tau_{c}>0$, all the three types of front are realizable numerically $[24,43,26]$.

To illustrate the numerical fronts, we present next some results obtained in [43], where a numerical scheme for the fully coupled barotropic-baroclinic-moisture equations was designed and implemented. We consider a situation where a moist region is surrounded by two dry regions that are separated but a drying front on the left, that moves at speed $c_{m}<s_{d}<c_{d}$ towards the moist region, and a slow moistening front on the right that moves at a slow speed $0<s_{m}<c_{m}$ towards the dry region. Because 
the drying front moves faster, the moistening front ultimately will be overtaken by the drying front and the moist region will disappear. This is illustrated in Figure 6 by a numerical simulation for the case of a finite relaxation time $\tau_{c}=2$ hours and with the theoretical solution when $\tau_{c} \longrightarrow 0$. The initial condition is set to the limiting solution for both models.

When linear extrapolation boundary conditions are used instead, the limiting solution is extrapolated outside the computational domain. In this case, the numerical solution with $\tau_{c}=2$ hours lies on top of the theoretical limit as shown on Figure 6 (B). This in particular demonstrates that both the drying and the slow moistening fronts are realized numerically. However, when periodic boundary conditions are used, interesting things happen, as shown on Figure $6(\mathrm{C})$. The periodic boundary condition induces a jump in the solution that triggers two dry gravity waves that move towards the moist region. As they hit the precipitation fronts on each side of the moist region, one of them initiates a fast moistening front that moves backwards while the other completely sweeps the moist region. We note that the fast moistening front was not forced externally but initiated freely from the interaction of a gravity wave and a drying front. Thus, this makes a strong case for the stability and realizability of both moistening fronts in the limit $\tau_{c} \longrightarrow 0$, and recent mathematical theory justifies this claim [52].

Similar systems of equations have also been studied by [53, 54], in which further interesting issues such as front formation are investigated.

\subsubsection{Analytic formulation of moisture front solution with finite relaxation time, $\tau_{c}>0$}

Here we follow [26] to construct analytical moisture-front solutions when the relaxation time $\tau_{c}$ is non zero. Inspired by the numerical simulations in [24] and [43], it is natural to assume a solution ansatz in the scaled variable $(x-s t) / \tau_{c}$ :

$$
w=\left\{\begin{array}{cl}
w^{-} & \text {in the dry region, where } P=0 \\
f\left(\frac{x-s t}{\tau_{c}}\right) & \text { in the moist region, where } P>0 \\
w^{+} & \text {as } x \longrightarrow+\infty .
\end{array}\right.
$$

where $w^{ \pm}$are fixed constants to be determined, and where a similar ansatz is used for $\theta_{x}$ and $q_{x}$. For simplicity we introduce the variable $\tilde{x}=(x-s t) / \tau_{c}$, and we assume that the dry region is on the left of $\tilde{x}=0$ and the moist region is on the right. By inserting this ansatz into (3.25), a single ODE can be derived for the variable $Z_{x}=q_{x}+\tilde{Q} \theta_{x}$ in the moist region:

$$
\frac{d Z_{x}}{d \tilde{x}}=-a Z_{x}-b
$$

where $a=-\frac{1+\alpha}{s} \frac{c_{m}^{2}-s^{2}}{1-s^{2}}$ and $b$ are constants. A simple integration leads to analytical solutions of smoothed precipitation fronts for non-zero $\tau_{c}$. In the moist region,

$$
\begin{aligned}
& w=w^{+}-[w] \exp \left(-a \frac{x-s t}{\tau_{c}}\right) \\
& P=P^{+}\left[1-\exp \left(-a \frac{x-s t}{\tau_{c}}\right)\right],
\end{aligned}
$$



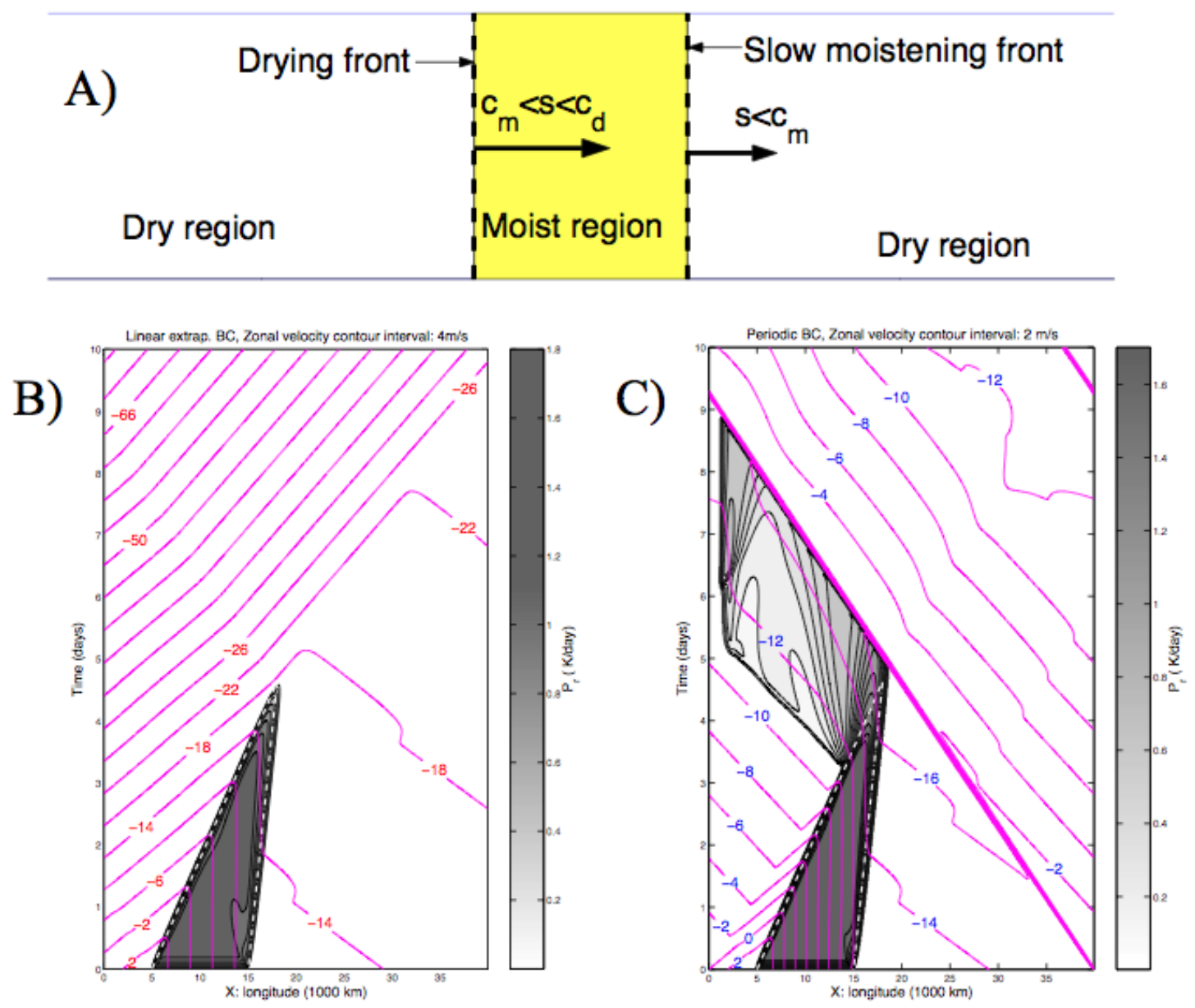

Figure 6. Evolution of a dying moisture wave. (A) Sketch of initial setup consisting of a moist region in grey surrounded by two drying regions. Indicated by dashed lines are a drying front on the left that moves at speed $c_{m}<s_{d}<c_{d}$ towards the moist region and a slow moistening front on the right that moves at a slow speed $0<s_{m}<c_{m}$ towards the drying. (B) Contours in the (x,t) domain of zonal velocity and filled contours of precipitation showing the evolution of the dry moisture wave for a finite relaxation time $\tau_{c}=2$ hours and linear continuation boundary conditions are used. (C) Same as (B) but with periodic boundary conditions. The thick dashed line in (B) represents the theoretical limiting-dynamics precipitation fronts. Adapted with permission from [43]. 

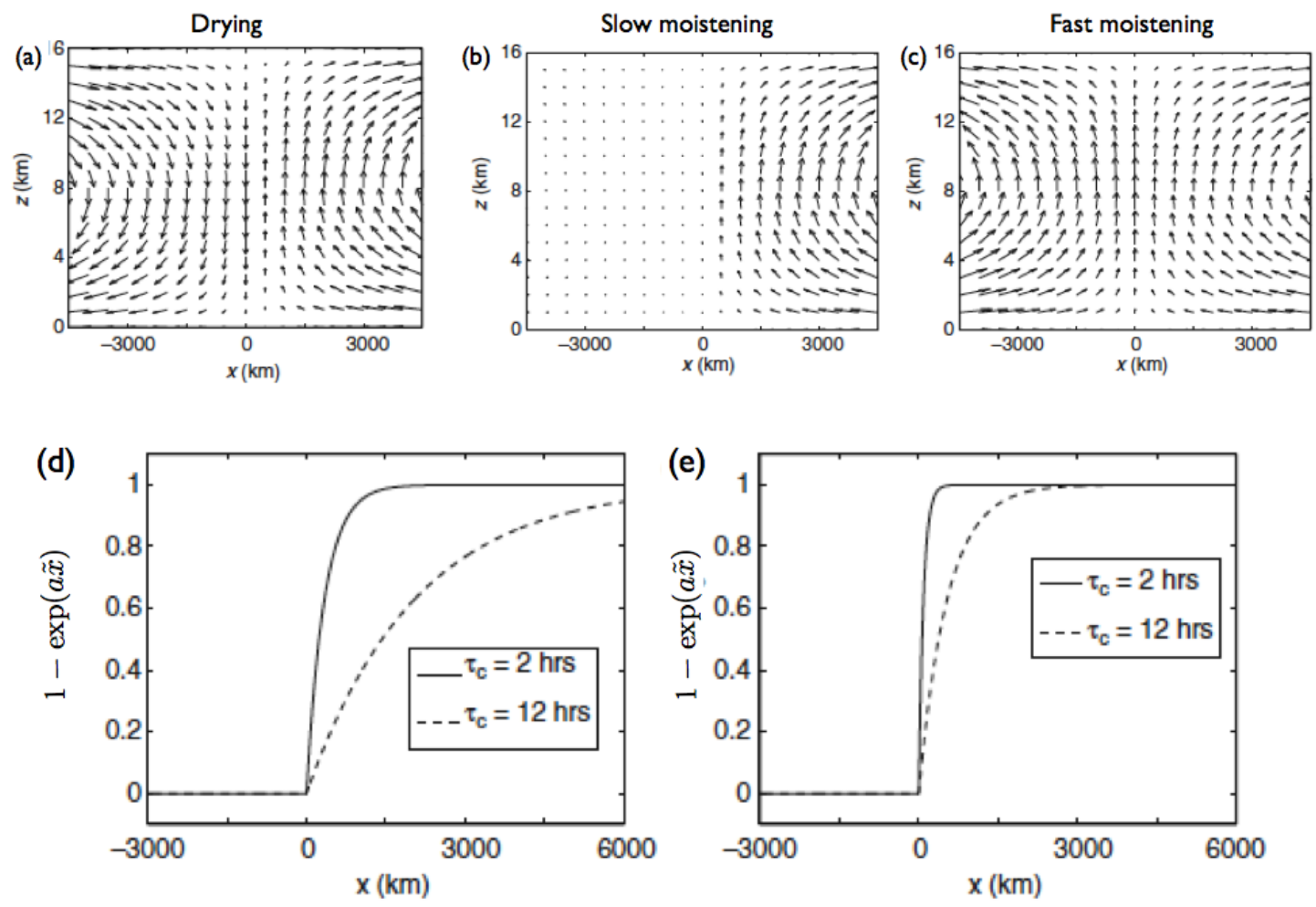

Figure 7. Velocity field for the (a) drying, (b) slow moistening, and (c) fast moistening fronts at finite relaxation time $\tau_{c}=2$ hours. Precipitation profile showing the width of the boundary region between the dry region and the far field moist region for $\tau_{c}=2$ hours and $\tau_{c}=12$ hours with $\tilde{Q}=0.9, \alpha=0$ while (d) $s=-4 \mathrm{~m} \mathrm{~s}^{-1}$ and (e) $s=-1$ $\mathrm{m} \mathrm{s}^{-1}$. Adapted from [26]

with similar formulas for $\theta_{x}$ and $q_{x}$. In order to have solutions that do not grow exponentially, we impose the constraint $a>0$. As a result, the allowed fronts are exactly the three types in (3.29) that were found for the limiting case $\tau_{c} \longrightarrow 0$ : $c_{m}<s<1,-c_{m}<s<0$, and $s<-1$, corresponding, respectively, to a drying front, a slow moistening front, and a fast moistening front [26]. The flow fields associated with each one of these three cases are illustrated in Figure 7 for the typical value $\tau_{c}=2$ hours.

The exponential folding distance, $L_{c}=\tau_{c} / a$, determines the steepness of the interface between the dry region and the far field moist region. This depends not only on $\tau_{c}$ (it increases with $\tau_{c}$ ) but also on the parameters $\tilde{Q}, \alpha$ and the front speed s. To illustrate this, we show on the bottom of Figure 7 the precipitation profile $\left[1-\exp \left(-a \frac{x-s t}{\tau_{c}}\right)\right]$ through the interface. The choice of these parameters determines the width of the front and thus its acceptability as a physical wave.

3.2.5. Wave reflection and transmission at the interface of a moisture front Given that we have different types of waves within the moist and dry regions, it is interesting to 
(a)

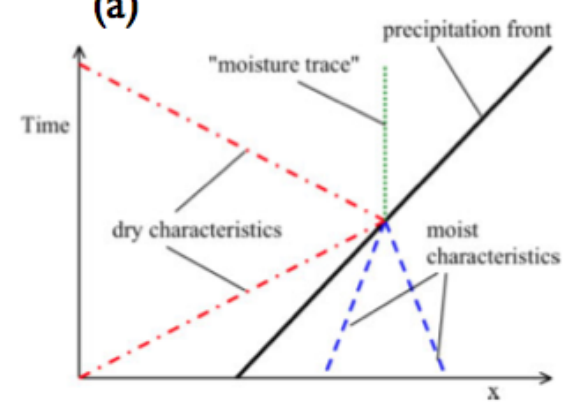

(b)

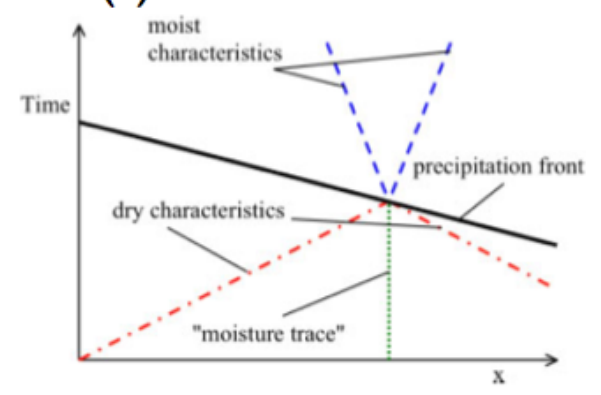

(c)

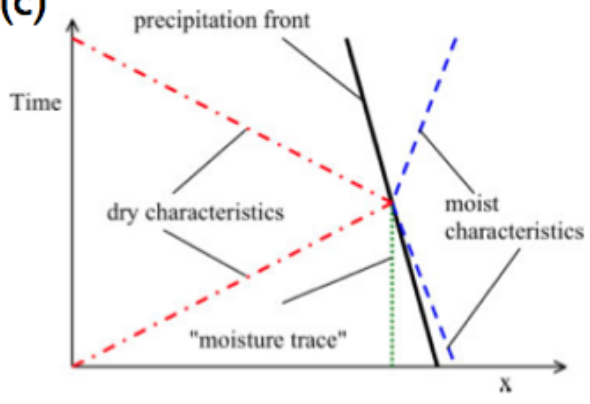

Figure 8. Schematic representation of the interactions of the dry and moist characteristics with the precipitation front. (A) Drying, (B) slow moistening, and (C) fast moistening. In each case, three incoming waves yield two transmitted and/or reflected waves of different nature. Adapted from [55].

investigate the interaction of small disturbances with the moisture fronts. The sudden change in propagation speed may result in the phenomena of wave reflection and wave transmission just like in optics. This is studied in [55].

We begin by introducing Riemann invariants within both the dry and moist regions. Within the dry region, there are three invariants, which are associated with eastward and westward moving gravity waves and a standing moisture mode. They are given in terms of the gradient ( $x$-derivatives) of (3.25) by [55]

$$
A_{d}^{+}=w+\theta_{x}, A_{d}^{-}=w-\theta_{x}, \text { and } A_{d}^{0} \equiv Z_{x}=q_{x}+\tilde{Q} \theta_{x},
$$

which form, respectively, westward and eastward gravity waves, moving at the dry gravity wave speed, $\pm c_{d}= \pm 1$, and a standing moisture mode (or a moisture trace to rephrase [55]). Within the moist region, however, the PDE system (3.25) is overdetermined because of the constraint $q_{x}=\alpha \theta_{x}$. However, as already done earlier, a moist gravity wave system is obtained by "averaging" the $\theta_{x}$ and $q_{x}$ equations to obtain $(1+\alpha)\left(\theta_{x}\right)_{t}+(1-\tilde{Q})\left(\theta_{x}\right)_{x}=-(1-\alpha) P_{x}$. Thus, only the two moist gravity waves moving at the moist gravity wave speed $\pm c_{m}= \pm[(1-\tilde{Q}) /(1+\alpha)]^{1 / 2}$ are present as Riemann invariants. They are given by [55]

$$
A_{m}^{+}=w+\frac{1}{c_{m}} \theta_{x} \text { and } A_{m}^{-}=w-\frac{1}{c_{m}} \theta_{x} .
$$

Easy algebraic manipulations lead to the expression of the moisture front solutions in (3.28)-(3.29) in terms of these Riemann invariants [55].

Interestingly, for each case, we have a system of six unknowns and three equations, namely, the front speed, $s$, and the five Riemann invariants, and the three jump conditions (3.27), respectively. Therefore, given three incident Riemann invariants (or waves), which admit a precipitation front of a certain type, the speed $s$ and two emanating waves are uniquely determined and computed from those formulas [55]. An 

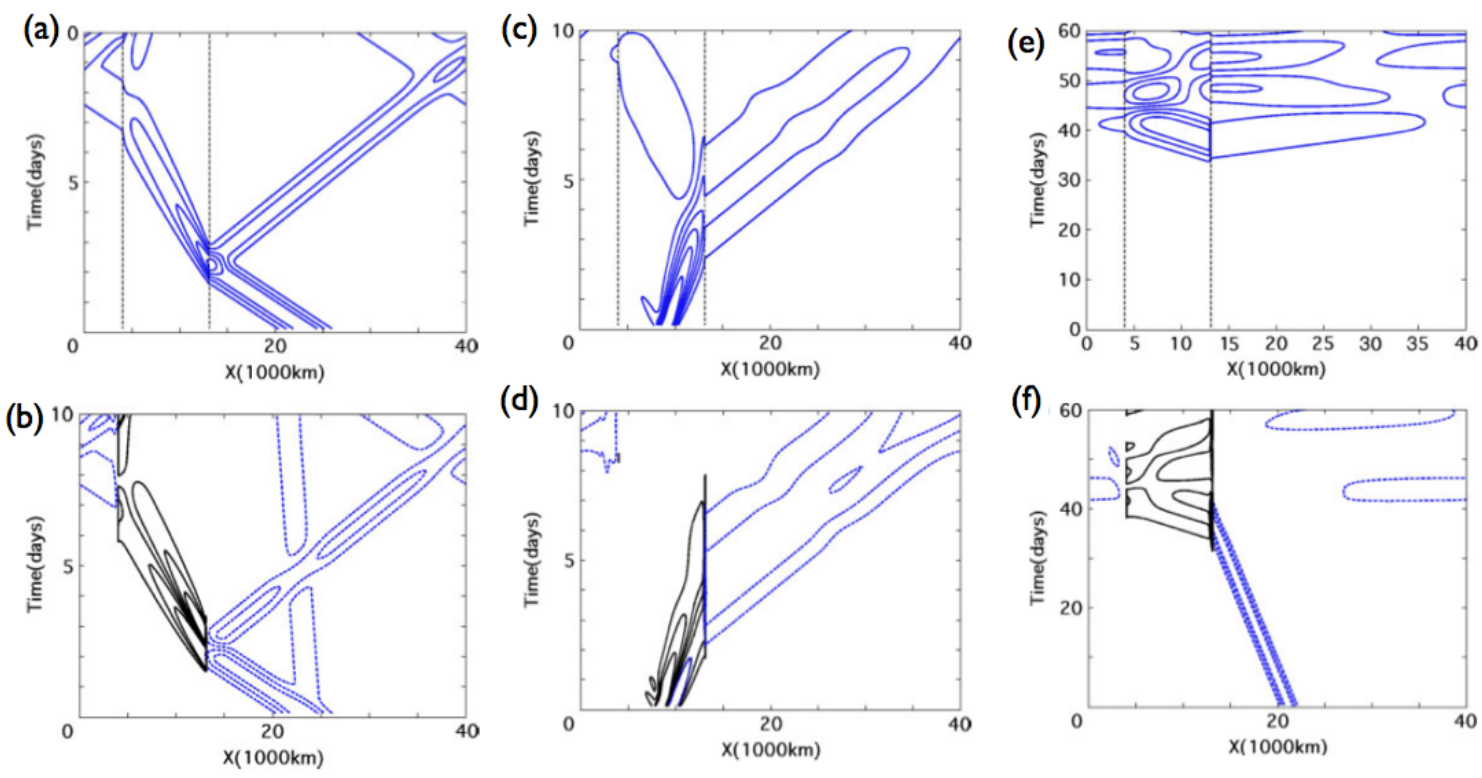

Figure 9. Numerical simulations of the evolution of perturbed slow moistening front. $(\mathrm{A})$ and $(\mathrm{B})$ : westward moving $\left(A_{d}^{+}\right)$dry-disturbance. $(\mathrm{C})$ and $(\mathrm{D})$ Eastward moving $\left(A_{m}^{-}\right)$moist-disturbance. $(\mathrm{E})$ and $(\mathrm{F})$ Standing (moisture mode, $A_{d}^{0}$ ) disturbance. Contours of the zonal wind and moisture (within the dry region) and precipitation (within the moist region) and shown on the top and bottom panels, respectively. The thin dashed lines delimit the moist region of the background. Adapted from [55].

illustrative sketch of the associated characteristics is given in Figure 8 for each one of the three front types.

Now we investigate the behavior of a small perturbation, in the initial data, on the form of an incident wave, of an already established moisture front background solution. This is somewhat in the spirit of the experiment in Figure 6 (C) but with a much smaller perturbation that would not destroy and/or change the behavior (type) of the background moisture front.

Following [55], we write the functional form of the emanating waves in terms of the incident waves as

$$
A_{\text {out }, k}=F_{k}\left(A_{i n, 1}, A_{\text {in }, 2}, A_{\text {in }, 3}\right), k=1,2 .
$$

Now, assume $A_{i n, l}=A_{i n, l}^{0}+\delta A_{i n, l}^{1}$ for $l=1,2,3$ a background value correspondent to the well-established front, and a small perturbation. Here $\delta>0$ is a small amplitude parameter. As consequence, we have

$$
A_{\text {out }, k}=A_{\text {out }, k}^{0}+\delta A_{\text {out }, l}^{1}+O\left(\delta^{2}\right), k=1,2 .
$$

A simple Taylor expansion then yields

$$
A_{\text {out }, k}^{1}=\sum_{l=1}^{3} \frac{\partial F_{k}}{\partial A_{i n, l}} A_{i n, l}^{1} .
$$


In [55], analytic expression for the coefficients $\partial F_{k} / \partial A_{l}$ are computed from the formulas for the emanating waves.

Three numerical simulations of the evolution of such perturbations to a background precipitation front solution are presented in [55] in the case of a slow moistening front. They are summarized in Figure 9. In Panels (A) and (B), we have an incident dry gravity wave disturbance that 'splits' into a reflected dry gravity wave and a transmitted moist gravity wave when it hits the slowly moving front with $\bar{U}=-s=-5 \mathrm{~m} / \mathrm{s}$. In Panels (C) and (D), we see an incident moist gravity wave that is also reflected and transmitted. Panels (E) and (F) relate to a standing-moisture mode that is reflected in a form of a dry gravity wave and transmitted to the moist region as a moist-gravity wave, when it first hits the front. The transmitted moist gravity wave ultimately hits the left boundary of the moist region, though not a sharp front, and starts bouncing back and forth within the moist region. The pictures in Figure 9 can be regarded as lying in a frame moving with the speed of the moistening front.

\section{Scale-dependent and multiscale models}

The tropics are home to a rich variety of scale-dependent dynamics. As a basis for comparison with the tropics, midlatitude balanced dynamics are governed primarily by quasi-geostrophy, which arises due the rapid rotation at those latitudes in association with a single length scale, the Rossby deformation radius. In contrast, in the tropics, quasi-geostrophy breaks down because the Coriolis force vanishes at the equator. Consequently, there are several different types of balanced tropical dynamics, and they arise on different space and time scales. Moreover, it is not as easy to guess the balanced dynamics in the tropics. Nevertheless, systematic asymptotics can be used to derive the different scale-dependent dynamics in the tropics, and it can also reveal the multiscale interactions.

In this section, some specific examples of scale-dependent and multiscale models are described in some detail in sections 4.1 and 4.2. Then, in the remaining part of the section, an overview is given of models from the planetary scales down to sub-cloud scales.

\subsection{Two examples}

Two examples are described in some detail in this section: a scale-dependent model for balanced dynamics, and a multiscale model for interactions between synoptic and planetary scales.

The first model for balanced tropical dynamics was derived by Charney [56], and it has recently received more attention $[57,58,59]$, including the appellation of "weaktemperature-gradient" (WTG) approximation. The observational basis of such WTG approximations is the fact that horizontal temperature fluctuations are small in the tropics due to rapid adjustment by gravity waves. This particular WTG model is valid 
on the Charney inertial scales of $\approx 500 \mathrm{~km}$ and $\approx 1$ day. Since these scales fall within the realm of equatorial "mesoscales," this particular WTG model was called the mesoscale equatorial WTG (MEWTG) model by [59], who also present a derivation of the model using systematic asymptotics. (See [60] for another simpler derivation.) The resulting MEWTG equations take the form

$$
\begin{aligned}
& \frac{\partial \mathbf{V}}{\partial t}+\mathbf{V} \cdot \nabla \mathbf{V}+W \frac{\partial \mathbf{V}}{\partial z}+\beta y \mathbf{V}^{\perp}=-\nabla \Phi+S_{\mathbf{V}} \\
& \frac{\partial \Phi}{\partial z}=g \frac{\Theta}{\theta_{0}} \\
& \frac{N^{2} \theta_{0}}{g} W=S_{\Theta} \\
& \nabla \cdot \mathbf{V}+\frac{\partial W}{\partial z}=0
\end{aligned}
$$

Notice that the heat source $S_{\Theta}$ is in balance with adiabatic cooling/heating $\left(N^{2} \theta_{0} / g\right) W$ associated with vertical motions. These equations have the form of the primitive equations (3.1) with the "temperature gradient" terms neglected as higher-order terms. The MEWTG model describes flows that are balanced with respect to much faster gravity waves, which have been effectively filtered out. The flow is driven by the heat source $S_{\Theta}$, which generates horizontal convergence and/or divergence.

The MEWTG model (4.1) has been used to study many aspects of tropical dynamics, including the hurricane embryo [60, 61], as described further in section 4.3.5. In addition, in the special case with $S_{\Theta}=W=0$, a canonical balanced model is obtained for strongly stratified flow $[62,22]$, where the flow is purely horizontal but varies with height.

An interesting aspect of tropical dynamics is that different balanced dynamics arise on different space and time scales. While the MEWTG model above describes balanced dynamics on mesoscales, there are different WTG models on synoptic scales [synoptic equatorial-scale WTG (SEWTG)] and on slightly-smaller-than-planetary scales and intraseasonal-to-seasonal scales [seasonal planetary equatorial WTG (SPEWTG)]. These different WTG regimes were identified by Majda and Klein [59]. These and other dynamical regimes are described schematically in figure 10.

The balanced WTG dynamics can also have multiscale interactions with other scales. One example of this is the IPESD (intraseasonal planetary equatorial synopticscale dynamics) model of Majda and Klein 2003 [59], which involves interactions between 


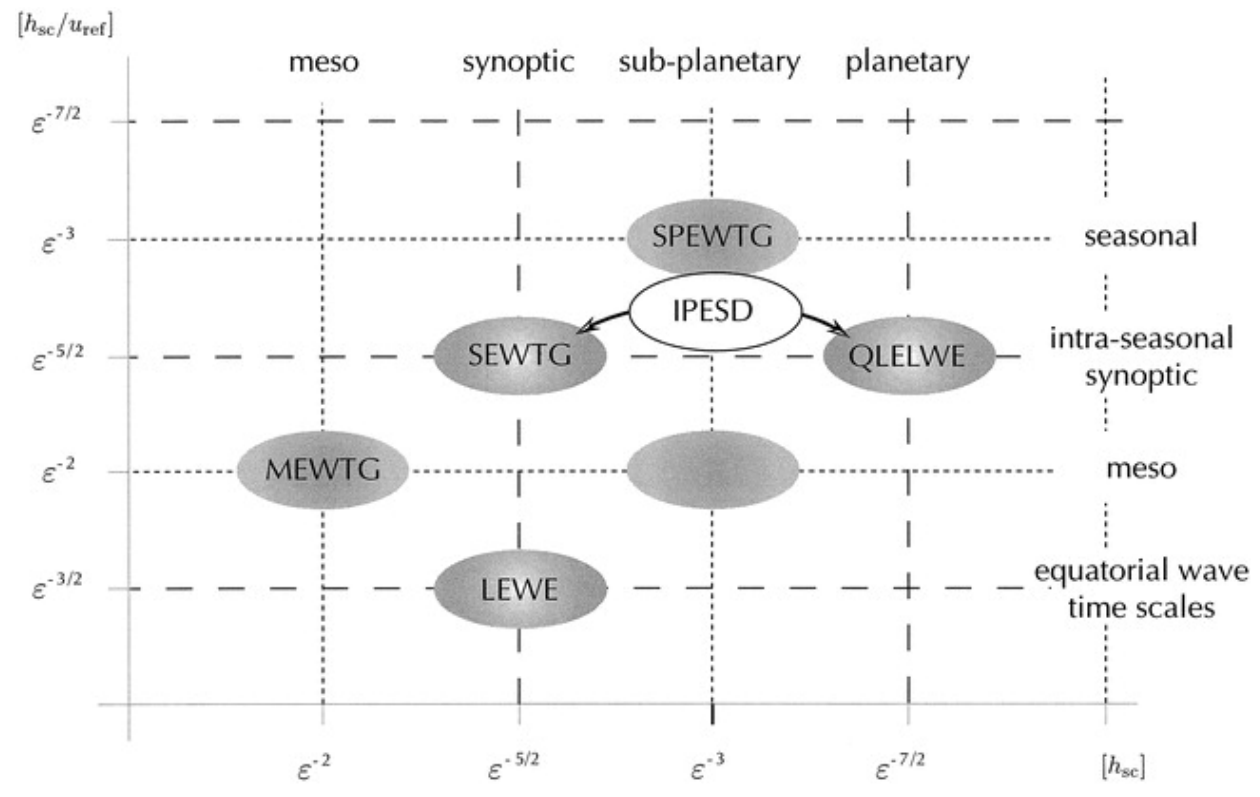

Figure 10. Scale-dependent and multiscale models for the tropics derived by [59] using systematic asymptotics.

the SEWTG balanced model on synoptic scales,

$$
\begin{aligned}
& -\beta y V^{\prime}=-\frac{\partial \Phi^{\prime}}{\partial x} \\
& \beta y U^{\prime}=-\frac{\partial \Phi^{\prime}}{\partial y} \\
& \frac{\partial \Phi^{\prime}}{\partial z}=g \frac{\Theta^{\prime}}{\theta_{0}} \\
& \frac{N^{2} \theta_{0}}{g} W^{\prime}=S_{\Theta}^{\prime} \\
& \frac{\partial U^{\prime}}{\partial x}+\frac{\partial V^{\prime}}{\partial y}+\frac{\partial W^{\prime}}{\partial z}=0
\end{aligned}
$$

and the quasi-linear equatorial long-wave equations (QLELWE) on intraseasonal/planetary scales,

$$
\begin{aligned}
& \frac{\partial \bar{U}}{\partial t}-\beta y \bar{V}=-\frac{\partial \bar{\Phi}}{\partial X}+\overline{S_{U}}-\frac{\partial}{\partial y} \overline{V^{\prime} U^{\prime}}-\frac{\partial}{\partial z} \overline{W^{\prime} U^{\prime}} \\
& \beta y \bar{U}=-\frac{\partial \bar{\Phi}}{\partial y} \\
& \frac{\partial \bar{\Phi}}{\partial z}=g \frac{\bar{\Theta}}{\theta_{0}} \\
& \frac{\partial \bar{\Theta}}{\partial t}+\frac{N^{2} \theta_{0}}{g} \bar{W}=\overline{S_{\Theta}}-\frac{\partial}{\partial y} \overline{V^{\prime} \Theta^{\prime}}-\frac{\partial}{\partial z} \overline{W^{\prime} \Theta^{\prime}} \\
& \frac{\partial \bar{U}}{\partial X}+\frac{\partial \bar{V}}{\partial y}+\frac{\partial \bar{W}}{\partial z}=0 .
\end{aligned}
$$

In the IPESD model, the large-scale envelope evolves according to the QLELWE, 
which are driven by planetary-scale heat sources $\overline{S_{\Theta}}(X, y, z, t)$ as well as upscale momentum and temperature fluxes from synoptic scales. This model extends the traditional Matsuno-Gill model [33, 63, 22] in two important ways: (i) it includes upscale fluxes from synoptic scales, and (ii) it allows a general vertical structure instead of a single vertical baroclinic mode. Application of this model to the structure of the MJO $[64,65]$ will be illustrated in section 5.6 below.

\section{2. "Self-similarity" in tropical convection}

A remarkable feature of organized tropical convection is that the coherent structures on three scales all have similar structures in the $x-z$ plane: squall lines on mesoscales, CCW on equatorial synoptic scales, and the MJO on planetary/intraseasonal scales [66]. Not only are the structures similar on these different scales, but each of the coherent structures is actually an envelope of the smaller-scale coherent structures: the MJO is an envelope of the smaller-scale CCW, which are, in turn, envelopes of smaller-scale convective systems on mesoscales. These features motivate the term "self-similarity" in tropical convection, where it is clear that this version of "self-similarity" is different from that which is usually associated with fractals and power laws [67].

While the above description is based on observational "self-similarity," it can also be described dynamically. Majda [68] has derived a series of multiscale models for mesoscales, synoptic scales, and planetary scales; and he has shown that the form of the dynamics - and the multiscale interactions - is the same on the mesoscale-synoptic-scale

set of scales as it is on the synoptic-planetary-scale set of scales, when viewed in the $x-z$ plane above the equator. In other words, each of the two multiscale models has the following form (in the $x-z$ plane above the equator): small-scale equations

$$
\begin{aligned}
& \frac{\partial U^{\prime}}{\partial \tau}+\frac{\partial \Phi^{\prime}}{\partial x}=0 \\
& \frac{\partial \Theta^{\prime}}{\partial \tau}+\frac{N^{2} \theta_{0}}{g} W^{\prime}=S_{\Theta}^{\prime} \\
& \frac{\partial \Phi^{\prime}}{\partial z}=\Theta^{\prime} \\
& \frac{\partial U^{\prime}}{\partial x}+\frac{\partial W^{\prime}}{\partial z}=0
\end{aligned}
$$

and large-scale equations

$$
\begin{aligned}
& \frac{\partial\langle\bar{U}\rangle}{\partial t}+\frac{\partial\langle\bar{\Phi}\rangle}{\partial X}=\left\langle\overline{S_{U}}\right\rangle-\frac{\partial}{\partial z}\left\langle\overline{W^{\prime} U^{\prime}}\right\rangle \\
& \frac{\partial\langle\bar{\Theta}\rangle}{\partial t}+\frac{N^{2} \theta_{0}}{g}\langle\bar{W}\rangle=\left\langle\overline{S_{\Theta}}\right\rangle-\frac{\partial}{\partial z}\left\langle\overline{W^{\prime} \Theta^{\prime}}\right\rangle \\
& \frac{\partial\langle\bar{\Phi}\rangle}{\partial z}=\langle\bar{\Theta}\rangle \\
& \frac{\partial\langle\bar{U}\rangle}{\partial X}+\frac{\partial\langle\bar{W}\rangle}{\partial z}=0 .
\end{aligned}
$$




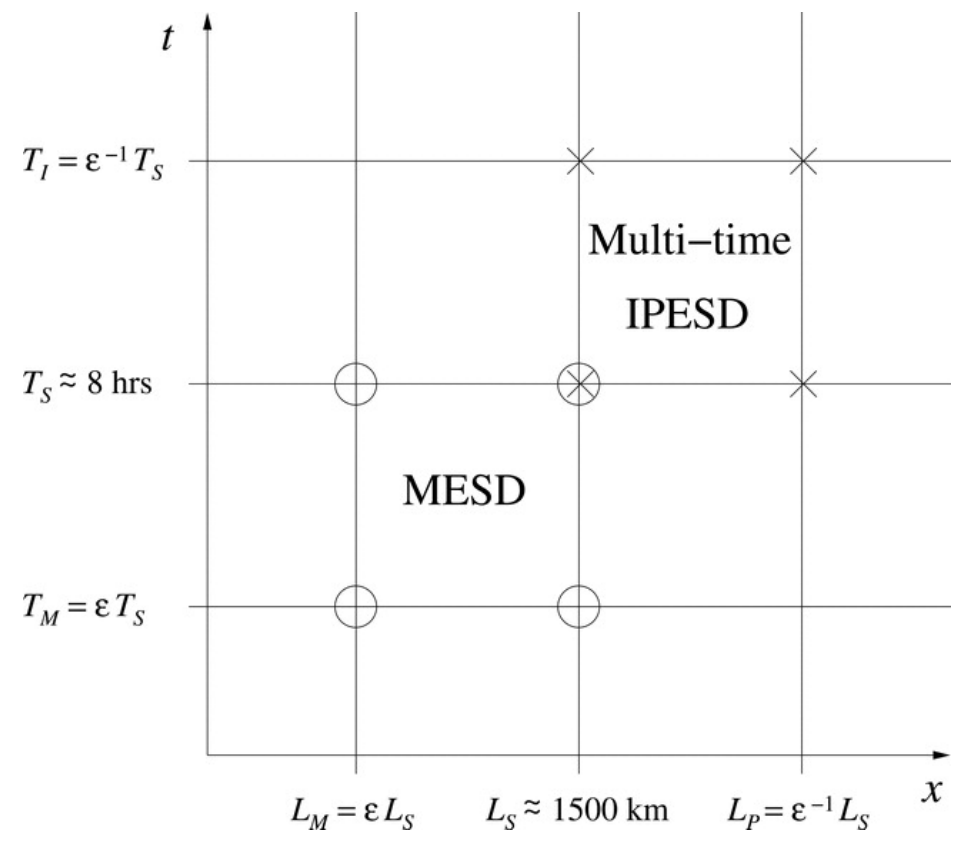

Figure 11. Scales described by the MESD (mesoscale equatorial synoptic dynamics) and multitime-scale IPESD models from [69].

This is a two-time-scale version of the IPESD model described earlier in (4.2)-(4.3), and it is also the same for the multiscale model of [68] that connects mesoscale and synoptic scales. These two sets of multiscale models are described schematically in figure 11, which shows the three "self-similar" sets of scales that are linked by the two multiscale models.

\subsection{From planetary to sub-cloud scales}

The tropics is replete with a variety of different phenomena on different scales. To complement the detailed examples given above, here we summarize the full range of scales, from planetary scales to scales within individual clouds, and the scale-dependent and multiscale models that describe them. For other overviews, see [70, 22, 71].

4.3.1. Moist dynamics on planetary scales In [72], planetary-scale equations are derived in a different setting than the planetary-scale equations described above from $[59,68]$. The main differences are that moisture is included and the Froude number is not required to be small, which allows for strong zonal winds such as Trade Winds and the Hadley Circulation. The strong zonal winds give rise to a multiscale interaction that was absent from the models described above: the strong zonal winds advect the smaller-scale fluctuations. While these models are appealing from a conceptual point of view, they are almost as complex as the original equations.

4.3.2. Convectively coupled waves and convective momentum transport A particularly successful application of multiscale models has been in the context of the MJO, 
convectively coupled waves (CCW), and convective momentum transport (CMT). The first multiscale asymptotic model applied in this context was the IPESD model, shown above in (4.2)-(4.3). Derived by Majda and Klein [59], it was applied to the MJO-CCWCMT problem by Majda and Biello [64, 65]. They showed that CMT due to CCW which enters the model as the terms $\overline{\left(v^{\prime} u^{\prime}\right)_{y}}$ and $\overline{\left(w^{\prime} u^{\prime}\right)_{z}}$ - are needed, in addition to mean heating $\overline{S^{\theta}}$, in order to reproduce some details of the MJO's structure.

While the IPESD model includes the one-way effect of CCW on the MJO in a transparent fashion, it does not include the opposite effect of the MJO on the CCW. These two-way interactions were both included in the dynamic model of [73] (although the full horizontal MJO structure was not included - only a single planetary scale column). In that model, the mean vertical wind shear determined which wave type was preferred - eastward- or westward-propagating - and hence the sign of the CMT as well. In this dynamic setting, it was shown that energy exchanges between the mean wind and the waves were sometimes downscale and sometimes upscale; i.e., the CMT from the CCW sometimes damped the mean wind and sometimes accelerated it. These interactions are further studied in $[74,75]$.

4.3.3. Superrotation The atmosphere is superrotating when the large-scale, vertically averaged, zonal equatorial winds move faster than the planetary rotation. Interestingly, in the tropics, the MJO has been observed to be superrotating [76]. According to Hide's Theorem, this observed superrotation implies that there necessarily must be upscale transports of momentum to sustain superrotation. Biello, Majda, and Moncrieff [77] use the multi-scale MJO models discussed here to show that the upscale fluxes from vertical transport of synoptic-scale momentum combined with positive planetary-scale heating drive this superrotation. In particular, there is no superrotation possible from planetary-scale heating alone in a Matsuno-Gill model; multi-scale effects are needed.

In addition, recent simulations suggest there may be enhanced MJO activity in a warmer climate, possibly leading to a superrotating tropical mean circulation [78].

4.3.4. Superparameterization Superparameterization is a multiscale computational technique for large-scale atmospheric models $[16,17,18]$. It involves coarse-scale and fine-scale models with grid spacings of roughly $200 \mathrm{~km}$ and $2 \mathrm{~km}$, respectively, and hence resolved dynamics on scales of roughly $2000 \mathrm{~km}$ and $20 \mathrm{~km}$, respectively. Significant computational savings are achieved by some simplifications in the fine-scale model: within each three-dimensional coarse-scale grid box, a two-dimensional fine-scale domain is used with periodic lateral boundary conditions. Pioneered by Wojciech Grabowski $[16,17]$, superparameterization has become a widely-used tool that yields results that are more realistic than traditional GCMs in many ways, including the MJO [18, 19, 15].

While much progress has been made with superparameterization, many challenges still remain. For example, while superparameterization gives a significant computational savings compared to using the fine grid spacing over the entire globe in three dimensions, it is still significantly more expensive than traditional global models with coarse grid 
spacings. New algorithms developed by Xing, Majda, and Grabowski [79] demonstrate ways to speed up superparameterization by a factor of 10 or even 30 while still retaining statistical accuracy. As another example, the theoretical underpinnings of superparameterization deserve further exploration. To this end, Majda [69] has derived equations for superparameterization using multiscale asymptotics, and Majda and Grote [80] have developed test models for understanding superparameterization and algorithms for it.

4.3.5. Hurricane embryo Hurricanes develop through different stages, and the hurricane embryo is a precursor stage before a fully-developed hurricane forms. In the embryo there is competition between different preconditioning mechanisms involving hydrodynamics and moist thermodynamics, which can lead to cyclogenesis [81]. Understanding this regime is very important for forecasting these extreme events. To sort out the competing factors in a simplified setting, Majda, Xing, and Mohammadian [61] have derived and studied multiscale models for the hurricane embryo. The model involves the interaction of three types of dynamics on three different spatiotemporal scales: a Balanced Mesoscale Vortex (BMV) model, a Balanced Hot Tower (BHT) model, and Moist Mesoscale Wave (MMW) model. The dynamical cores of both the BMV and BHT models are WTG models with similar structure as (4.1); they were studied in isolation from each other by Majda, Mohammadian, and Xing [60]. The multiscale model of [61] involves interactions between the different models through mechanisms such as upscale turbulent eddy fluxes and advection by the background wind. It was shown that this simplified model captures, in a qualitative fashion, several of the features observed in numerical simulations of comprehensive models for the hurricane embryo [81]. Furthermore, this multiscale model involves new PDE that merit rigorous mathematical study, as discussed further in section 8 below.

4.3.6. Squall lines Squall lines are organized cloud systems on mesoscales with deep convection aligned roughly on a straight line (segment) [82]. These coherent structures can sometimes transfer energy to the larger-scale flow through CMT [82], as described theoretically in, for instance, the multiscale models MESD and BMESD of Majda [68].

In addition, squall lines typically form in a strong background wind shear, and they typically have intense fluctuations (on scales of roughly $10 \mathrm{~km}$ ) within their organized mesoscale envelope (on scales of roughly $100 \mathrm{~km}$ ). Majda and Xing [83] have derived and studied a multiscale model that includes these multiscale aspects of squall lines. The form of the model involves a pair of time-dependent forced Taylor-Goldstein equations: a hydrostatic form for the mesoscale dynamics and a non-hydrostatic form for the fluctuation dynamics. Another difference between the pair is that the mesoscale dynamics are forced by turbulent eddy fluxes due to the fluctuations. Majda and Xing [83] solved the Taylor-Goldstein equations, and their results compared qualitatively with a comprehensive cloud resolving model simulation of a suite of squall lines. 
4.3.7. Moist thermodynamics and mesoscale convection Including moisture into multiscale models is a difficult challenge. Klein and Majda [84] have derived multiscale models for deep convection on mesoscales, and, significantly, they included an asymptotic analysis of a bulk microphysics parameterization of moist processes. This leads to separate treatments of regions of saturated and unsaturated air.

In a continuation of work with this model, Ruprecht, Klein, and Majda [85] were able to derive an exact mass flux formula by computing the weighted average over saturated and unsaturated regions. This can be thought of as homogenization over narrow cloudy towers, and the resulting key parameter is the "saturated area fraction" or "cloud area fraction." Again, it is emphasized that no closure approximations are required in this weighted average besides adopting the asymptotic limit regime on which the analysis is based. Then, using the new model, the effects of the moisture (i.e., saturated area fraction) were studied, and several changes from the dry case are identified such as moisture-induced critical layers and reduced gravity wave speeds due to moisture.

4.3.8. Shallow and deep cumulus clouds Finally, on small vertical scales, there are clouds in the moist atmospheric boundary layer, within a few kilometers of the surface. On these scales, the two most common types of clouds are shallow cumulus (which are common in the trades and elsewhere) and stratocumulus, both illustrated schematically in figure $1[12]$.

Multiscale models for shallow cumulus were derived by Stechmann and Stevens [86]. It was shown that, even on the scales of individual shallow cumulus clouds, the canonical balanced model (4.1) arises as the simplified dynamics for a balanced updraft. Moreover, a multiscale model showed that the balanced updraft is also forced by eddy fluxes from smaller-scale fluctuations such as pulses/thermals, which can be thought of as the coherent structures in this setting. These shallow cumulus models were also compared and contrasted with deep cumulus analogues, and the largest deep cumulus scales matched the smallest scales of $[61,84]$.

One important role of shallow cumulus is as a precursor to deep convection: they moisten and precondition the boundary layer and lower troposphere before deep convection $[12,87]$. In this context, it is often important to have a "bulk" representation of the moist boundary layer: by averaging over detailed fluctuations, one can obtain a simplified form that can be coupled with larger-scale models of deep convection. Stevens [87] provides an overview of bulk boundary layer models, including the assumptions needed in their derivation. As an application, Waite and Khouider [88] couple such a bulk boundary layer model with the multicloud model of Khouider and Majda [84]. They find that including the new boundary layer model enhances the instability of CCW compared to earlier versions of the multicloud model.

A different type of idealized model has been studied by Pauluis and Schumacher [89, 90] to understand atmospheric dynamics with a phase transition. The model is a moist analogue of Rayleigh-Bénard convection, with a piecewise linear equation of state to 


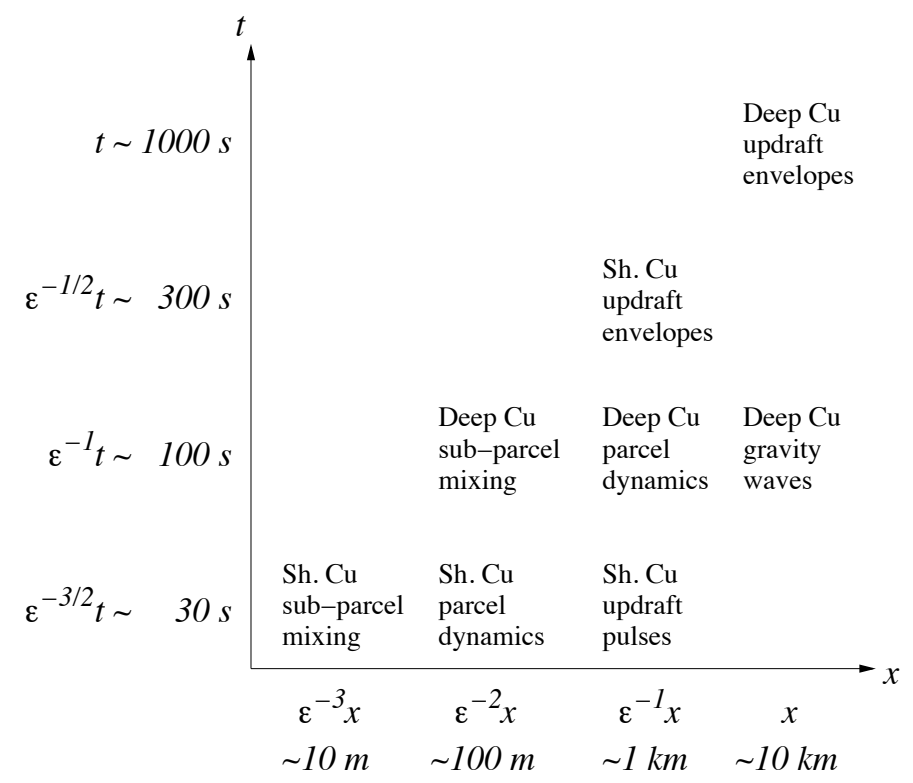

Figure 12. Scales involved in the scale-dependent and multi-scale models of shallow and deep cumulus cloud dynamics from [86].

represent saturated and unsaturated air. While the model is not derived systematically from the governing equations of the atmosphere, it provides a simple framework for studying fluid dynamics with a phase transition. This model has analogues of several moist convective regimes, including shallow cumulus and stratocumulus cloud decks and patches.

Multiscale models were derived by Owinoh, Stevens, and Klein [91] to understand interactions between boundary layer processes and mesoscale dynamics, with an eye towards the stratocumulus regime. These models could be used for studying the mesoscale organization of cloud-topped boundary layers.

\subsection{MJO skeleton model}

As mentioned above, the Madden-Julian Oscillation (MJO) is a planetary-scale wave envelope of complex multi-scale convection (see Figure 2). Originally discovered in the early 1970s, the MJO significantly affects monsoon development, intraseasonal predictability in midlatitudes, and the development of the El Niño southern oscillation (ENSO) in the Pacific Ocean, which is one of the most important components of seasonal prediction $[4,5]$.

Despite the importance of the MJO, computer simulations and theoretical understanding of it remain major challenges. Progress in these these areas is measured against the observed MJO, including its basic features of

I. slow eastward phase speed of $\approx 5 \mathrm{~m} / \mathrm{s}$,

II. peculiar dispersion relation of $d \omega / d k \approx 0$,

III. horizontal quadrupole vortex structure. 
These are the fundamental features of the MJO on intraseasonal/planetary scales, and they are referred to here as the MJO's "skeleton"; this is in contrast with additional features that are related to the smaller-scale features within the MJO envelope and referred to as the MJO's "muscle."

Recently, Majda and Stechmann [92, 93] introduced a minimal dynamical model that captures the MJO's intraseasonal/planetary scale features I-III, together, for the first time in a simple model. The model is a nonlinear oscillator model for the MJO skeleton and the skeleton of tropical intraseasonal variability in general. The fundamental mechanism of the model involves interactions between (i) planetary-scale, lower-tropospheric moisture anomalies and (ii) sub-planetary-scale, convection/wave activity (or, more precisely, the planetary-scale envelope of the sub- planetary-scale convective activity). These quantities are represented by the variables $\bar{Q}$ and $\bar{A}$, respectively:

$$
\begin{aligned}
& \bar{Q} \text { : lower tropospheric moisture anomaly, } \\
& \bar{A} \text { : amplitude of the convection/wave activity envelope. }
\end{aligned}
$$

The equations of the MJO skeleton model involve the QLELWE (4.3) for planetary/intraseasonal scale fluid dynamics, coupled with two additional equations for moisture $\bar{Q}$ and convective activity $\bar{A}$ :

$$
\begin{aligned}
& \frac{\partial \bar{U}}{\partial t}-\beta y \bar{V}=-\frac{\partial \bar{\Phi}}{\partial X} \\
& \beta y \bar{U}=-\frac{\partial \bar{\Phi}}{\partial y} \\
& 0=-\frac{\partial \bar{\Phi}}{\partial z}+g \frac{\bar{\Theta}}{\theta_{0}} \\
& \frac{\partial \bar{U}}{\partial X}+\frac{\partial \bar{V}}{\partial y}+\frac{\partial \bar{W}}{\partial z}=0 \\
& \frac{\partial \bar{\Theta}}{\partial t}+\frac{N^{2} \theta_{0}}{g} \bar{W}=H \bar{A}-\overline{S_{\Theta}} \\
& \frac{\partial \bar{Q}}{\partial t}-Q_{0} \bar{W}=-H \bar{A}+\overline{S_{Q}} \\
& \frac{\partial \bar{A}}{\partial t}=\Gamma \bar{Q} \bar{A} .
\end{aligned}
$$

The equation for $\bar{Q}$ is similar to that for $\bar{\Theta}$, and the dynamics of $\bar{A}$ is the simplest equation that embodies the following idea: positive low-level moisture anomalies create a tendency to enhance the envelope of convection/wave activity (and vice versa for negative moisture anomalies). Notice that this model contains a minimal number of parameters: $Q_{0}=0.9$, the (nondimensional) mean background vertical moisture gradient; and $\Gamma=1$, where $\Gamma \bar{Q}$ acts as a dynamic growth/decay rate of the wave activity envelope in response to moisture anomalies. In dimensional units, $\Gamma \approx 0.2 \mathrm{~d}^{-1} \mathrm{~K}^{-1}$. To obtain the simplest MJO skeleton model, truncated vertical and meridional structures are used to reduce (4.6) to dynamics with zonal $x$ and temporal variations only. 

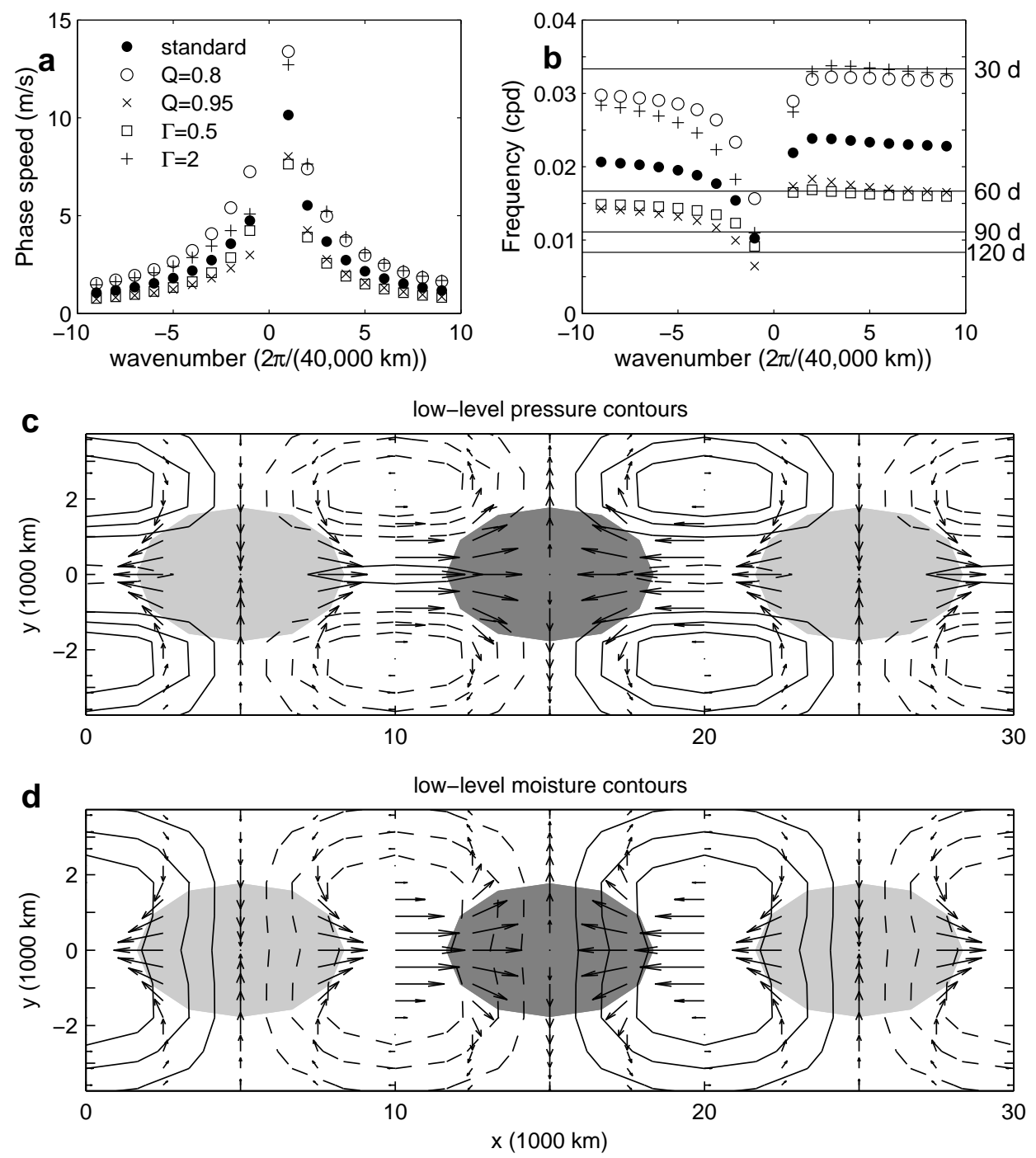

Figure 13. Linear theory for the MJO skeleton model. Phase speeds (a) and oscillation frequencies (b) for the two low-frequency mode types are shown as functions of the wavenumber. The westward- and eastward-propagating modes are displayed on the negative and positive branches of the wavenumber axis, respectively. Different symbols represent different choices of the model parameters. Adapted from [94, 92]. Horizontal structure of the wavenumber-2 MJO mode is shown with low-level pressure contours (a) and low-level moisture contours (b). Adapted from [94, 92].

The linear waves of (4.6) were investigated in [92], and a summary is shown in figure 13. For the two low-frequency mode types, the phase speeds and oscillation frequencies are shown in figures $13 \mathrm{a}$ and $13 \mathrm{~b}$, respectively. The westward- and eastward-propagating modes are displayed on the negative and positive branches of the wavenumber axis, respectively. The eastward-propagating mode is identified as the MJO mode through the fundamental features I-III above: slow eastward phase speed of $\approx 5 \mathrm{~m} / \mathrm{s}$ and an 

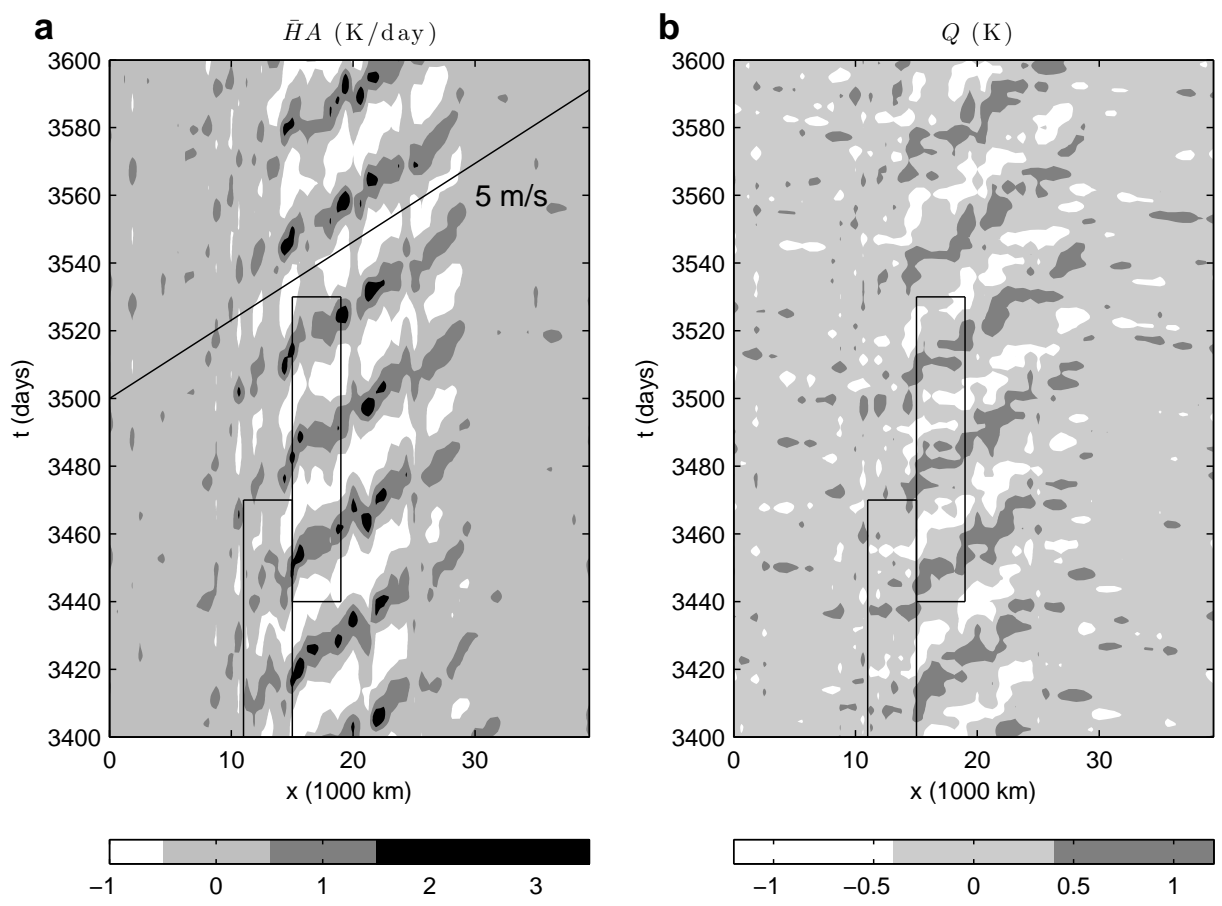

Figure 14. Nonlinear dynamics of the MJO skeleton model for a 200-day interval in a case with a warm-pool SST in the center half of the domain. (a) Anomalies of the convective activity. (b) Anomalies of the lower-tropospheric moisture. Adapted from [93].

oscillation frequency that is roughly constant and given by the simple formula

$$
\omega \approx \sqrt{\Gamma \overline{S_{\Theta}}\left(1-Q_{0}\right)}
$$

Furthermore, the MJO mode has a horizontal structure with quadrupole vortices, as shown in figures 13c,d and seen in observations. On the other hand, the westwardpropagating mode has a very low frequency that is essentially seasonal, not intraseasonal, for wavenumber 1 , and it has a much smaller contribution of moisture $\bar{Q}$ and convective activity $\bar{A}$ in comparison to the MJO mode [92].

The nonlinear dynamics of (4.6) were investigated in [93], and one case is shown in figure 14. This case has a background sea surface temperature (SST) with a warm pool in the center half of the domain, as a representation of the western Pacific warm pool. Over the 200-day interval shown, many MJO events can be seen with strong, irregular amplitudes over the warm pool and negligible amplitudes over the cold pool. Over the warm pool, these anomalies typically propagate slowly eastward at roughly 5 $\mathrm{m} / \mathrm{s}$. However, there are also localized instances of standing wave behavior, as can be seen within the two rectangular boxes. 


\section{Multicloud and multiscale models}

In this section, we present linear wave solutions and non-linear simulations for the primitive equations when the multicloud model is used for the parameterization of organized tropical convection. For the nonlinear simulations, we will consider both the simple case of reduced dynamics over the equator and the more general case using a general circulation model. We demonstrate, in particular, that the so-called multicloud model is capable of reproducing many key dynamical and physical features of the observed large scale convectively coupled waves, including the MJO. We will also expand some of the multiscale modeling ideas from the previous sections to the moist case to study the self-similarity across scales of tropical convective systems.

\subsection{The multicloud model}

Unlike the simple one-vertical-mode models discussed in section 3.2 above, the multicloud model is based on two vertical modes and on three cloud types, instead of one. To help the visualization, a cartoon of the cloud types and vertical modes is shown in Figure 15. The vertical modes are the first and second baroclinic modes introduced in section 3. The first baroclinic mode is heated by deep convection, much like the simple one-heating-mode model of section 3.2, and the second baroclinic mode is heated by both congestus clouds and stratiform clouds. Congestus clouds are observed to lead tropical convective systems and precondition the environment prior to deep convection. They heat the lower troposphere through condensation and induce a cooling of the upper troposphere through longwave radiation. Stratiform clouds, on the other hand, are observed to trail behind deep convection, and they (i) heat the upper troposphere as deep clouds enter their freezing phase and (ii) cool the lower troposphere due to the evaporation of stratiform rain that falls into the already dry environment.

The minimal dynamical core for the multicloud model consists of two coupled (linear) shallow systems: forced and coupled through heating rates associated with the three cloud types:

$$
\begin{aligned}
& \frac{\partial \mathbf{v}_{j}}{\partial t}+\beta y \mathbf{v}_{j}^{\perp}-\nabla \theta_{j}=-C_{d}\left(u_{0}\right) \mathbf{v}_{j}-\frac{1}{\tau_{W}} \mathbf{v}_{j}, j=1,2, \\
& \frac{\partial \theta_{1}}{\partial t}-\operatorname{div} \mathbf{v}_{1}=\frac{\pi}{2 \sqrt{2}} P+S_{1} \\
& \frac{\partial \theta_{2}}{\partial t}-\frac{1}{4} \operatorname{div} \mathbf{v}_{2}=\frac{\pi}{2 \sqrt{2}}\left(-H_{s}+H_{c}\right)+S_{2},
\end{aligned}
$$

where the two modes $j=1$ and 2 are coupled through the nonlinear source terms. These equations are essentially the same as (3.16)-(3.17) with the non-linear advection terms neglected and with heating rates $H_{c}, H_{d}, H_{s}$ that are interactive and associated with the three cloud types. The other source terms represent radiative cooling

rates: $S_{j}=-Q_{R, j}^{0}-\frac{1}{\tau_{R}} \theta_{j}, j=1,2$. Following [50], the total precipitation is set to $P=H_{d}+\xi_{s} H_{s}+\xi_{c} H_{c}$, and it includes contributions from deep convection, stratiform, and congestus clouds, and where $\xi_{s}$ and $\xi_{c}$ are parameters between 0 and 1. 

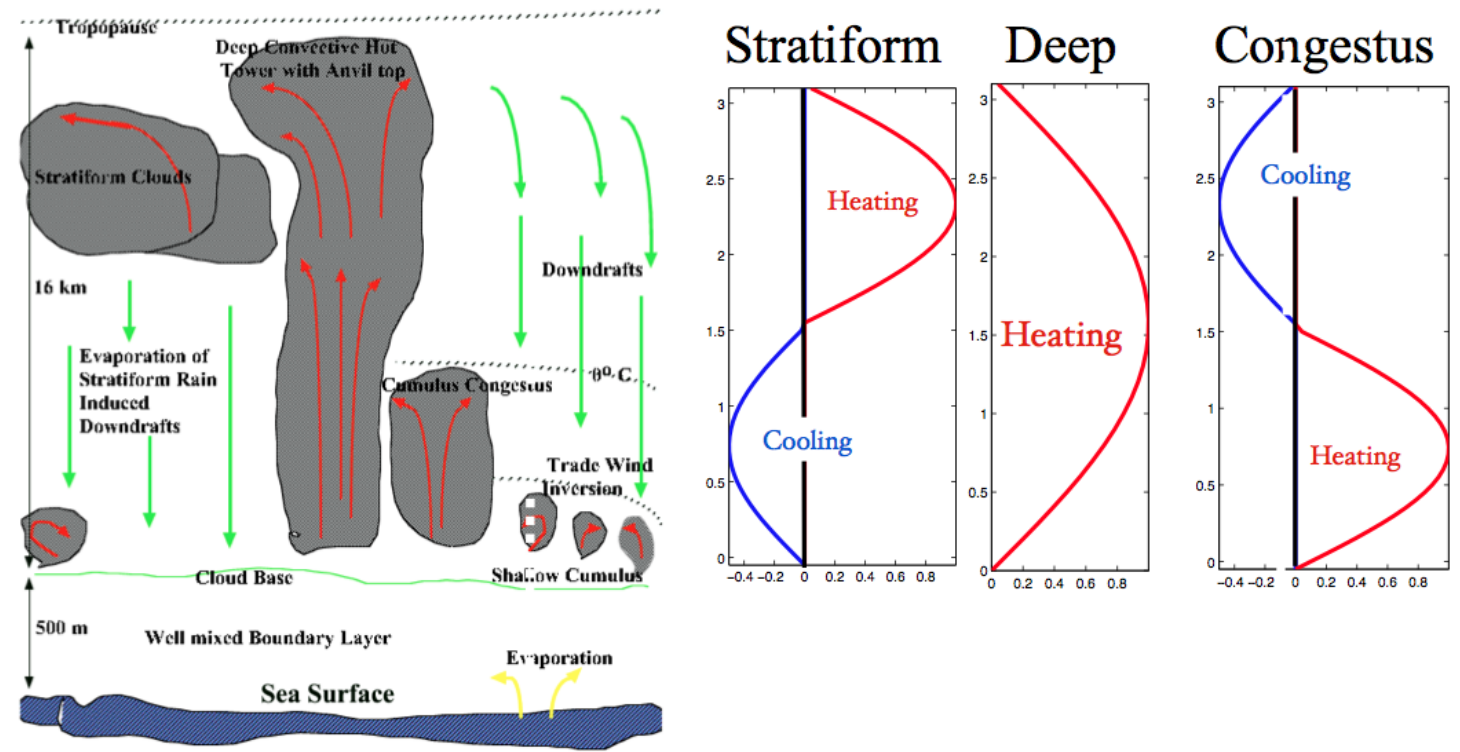

Figure 15. Left: Schematic of the three tropical cloud types interacting with the wellmixed planetary boundary layer above the sea surface through convective updrafts and downdrafts: the trade wind inversion, the freezing level with temperature $0^{\circ} \mathrm{C}$, and tropopause layers are shown. Right: Vertical profiles of heating and cooling fields associated with the three cloud types. Adopted from [50].

In addition to this dynamical core (5.1), there are equations for the boundary layer equivalent potential temperature, $\theta_{e b}$, and the vertically integrated moisture content, $q$ :

$$
\begin{aligned}
& \frac{\partial \theta_{e b}}{\partial t}=\frac{1}{h_{b}}(E-D) \\
& \frac{\partial q}{\partial t}+\operatorname{div}\left(\left(\mathbf{v}_{1}+\tilde{\alpha} \mathbf{v}_{2}\right) q\right)+\tilde{Q} \operatorname{div}\left(\mathbf{v}_{1}+\tilde{\lambda} \mathbf{v}_{2}\right)=-P+\frac{1}{H_{T}} D
\end{aligned}
$$

Here, $h_{b} \approx 500$ meters is the height of the moist boundary layer while $\tilde{Q}, \tilde{\lambda}$, and $\tilde{\alpha}$ are parameters associated with a prescribed moisture background and perturbation vertical profiles. According to the first equation in (5.2), $\theta_{e b}$ changes in response to the downdrafts, $D$, and the sea surface evaporation $E$. Like the simple-one heating model presented above, the equations in (5.2) are derived under the general constraint of conservation of vertically integrated moist-static energy for the fully coupled multicloud model. The moisture equation (5.2) follows from (3.20) in the same fashion as (3.21) but with both first and second baroclinic velocity components being retained. The parameter $\tilde{\lambda}$ measures the strength of moisture convergence due to the second baroclinic mode, and it plays an important role in the dynamics of convectively coupled tropical waves.

The main nonlinearities of the model are in the source terms $H_{d}, H_{c}, H_{s}$, and $D$. The stratiform and congestus heating rates, $H_{s}$ and $H_{c}$, satisfy relaxation-type equations:

$$
\frac{\partial H_{s}}{\partial t}=\frac{1}{\tau_{s}}\left(\alpha_{s} H_{d}-H_{s}\right)
$$


and

$$
\frac{\partial H_{c}}{\partial t}=\frac{1}{\tau_{c}}\left(\alpha_{c} \frac{\Lambda-\Lambda^{*}}{1-\Lambda^{*}} Q_{c}-H_{c}\right) .
$$

The deep convective heating, $H_{d}$, and the downdrafts, $D$, are given diagnostically by

$$
H_{d}=\frac{1-\Lambda}{1-\Lambda^{*}} Q_{d} \quad \text { and } \quad D=\Lambda D_{0} .
$$

The "moisture switch" function $\Lambda$ controls the transition between congestus to deep convection, and it depends on the difference between boundary layer and midtropospheric equivalent potential temperatures, $\theta_{e b}-\theta_{e m}$. The diagnostic functions $Q_{d}, Q_{c}, D_{0}$, and $\Lambda$ all involve nonlinear switches, and they are described in detail in [50]. As an example, $Q_{d}$ takes the form

$$
Q_{d}=\left[\bar{Q}+\frac{1}{\tau_{\text {conv }}}\left(a_{1} \theta_{e b}^{\prime}+a_{2} q^{\prime}-a_{0}\left(\theta_{1}^{\prime}+\gamma_{2} \theta_{2}^{\prime}\right)\right)\right]^{+}
$$

Here and elsewhere in the paper, $X^{+} \equiv \max (X, 0)$ is the nonlinear switch, unless otherwise specified. Finally, note that these source terms take slightly different forms in different versions of the multicloud model such as [49] and [50].

\subsection{Convectively coupled equatorial waves in the multicloud model}

In this section, we exhibit some typical solutions of the multicloud model equations (5.1)-(5.6) in the form of linear waves and long-time non-linear simulations.

For the linear waves, the base state is a state of radiative-convective equilibrium (RCE). RCE is a homogeneous (in space and time) steady state solution for the governing equations (5.1)-(5.6) around which waves grow and oscillate. In the multicloud model, an RCE solution is determined by fixing three important climatological parameters, the long-wave radiative cooling rate $Q_{R, 1}^{0}$, the discrepancy between the boundary layer equivalent potential temperature and its saturation value, $\theta_{e b}^{*}-\bar{\theta}_{e b}$, and the discrepancy between the boundary layer and midtropospheric equivalent potential temperatures, $\bar{\theta}_{e b}-\bar{\theta}_{e m}$. They are fixed according to climatological values that are recorded in the tropics $[49,50]: Q_{R, 1}^{0}=1 \mathrm{~K} \mathrm{day}^{-1}, \theta_{e b}^{*}-\bar{\theta}_{e b}=10 \mathrm{~K}$ while

$10 \leq \bar{\theta}_{e b}-\bar{\theta}_{e m} \leq 20 \mathrm{~K}$ or higher/lower according to whether we want to study a case of a dry or a moist middle troposphere. More details on the construction of climatologically sound RCE solutions for the multicloud model are found in [49, 44, 50].

The PDE system (5.1)-(5.6) is then linearized around the relevant RCE solution and linear solutions are sought with the form $U(x, t)=\hat{U} \exp [i(k x-\omega t)]$, where $U$ is the vector of diagnostic variables. Here $k$ is the zonal wavenumber and $\omega=\omega(k)$ is the generalized phase determined as an eigenvalue of the corresponding linear system, for a fixed value of $k$, while $\hat{U}(k)$ is the associated eigenvector. The real part of $\omega(k)$ defines the phase speed of the wave solution: $c(k)=\Re(\omega(k)) / k$ while the imaginary part represents its growth rate.

Before proceeding to the study of linear wave solution to the PDE system, it is important to select RCE's that are stable to small perturbation. This is easily achieved 
by looking at solutions of the linear system when $k=0$, which essentially represents solutions to the zonally averaged linear solutions. As shown in [49] and [44], this system exhibits interesting bifurcation behavior with respect to the model parameters. A typical stability diagram of the background RCE solution with respect to the parameters $\alpha_{c}, \alpha_{2}$ and $\bar{\theta}_{e b}-\bar{\theta}_{e m}$ is shown in Figure 16. Such diagrams are used as guidelines to select the appropriate parameters for the multicloud model $[49,44,50]$. This in particular results in interesting dynamics of the associated (nonlinear) ODE system that exists some periodic and/or chaotic solutions in various regimes of the parameters [44] as the system is dynamically driven back and forth between the stable and unstable regions.
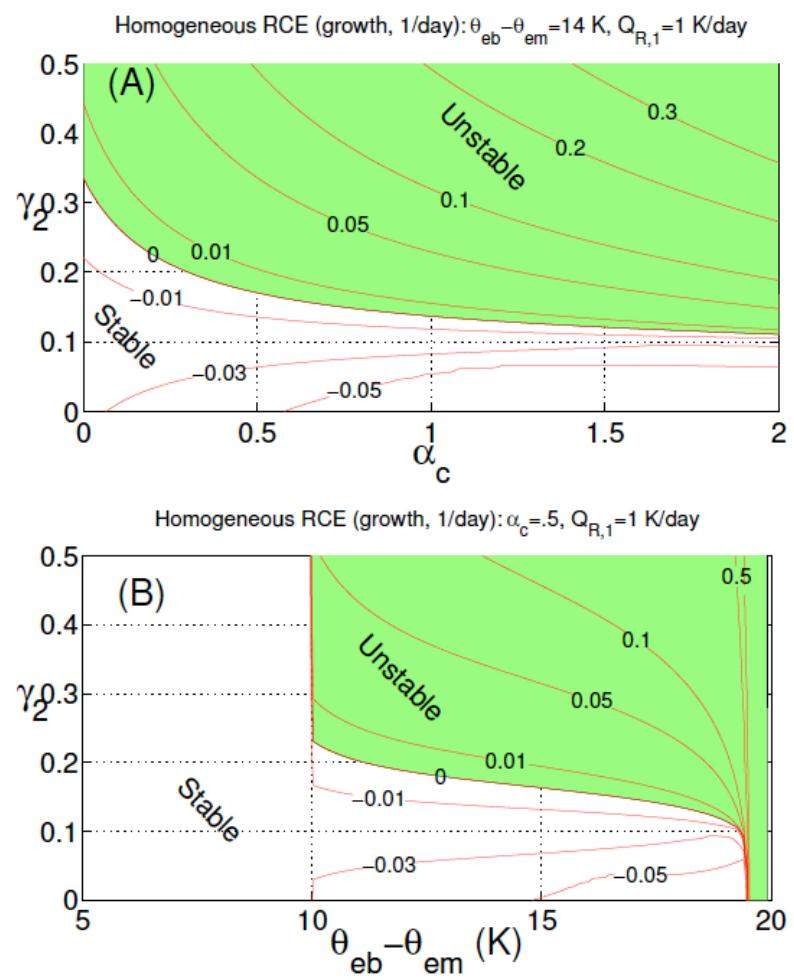

Figure 16. Bifurcation diagram for the homogeneous state RCE (a) in the $\gamma_{2}-\alpha_{c}$ plane for fixed $\bar{\theta}_{e b}-\bar{\theta}_{e m}=14 \mathrm{~K}$ and (b) in the $\gamma_{2}-\bar{\theta}_{e b}-\bar{\theta}_{e m}$ plane for fixed $\alpha_{c}=.5$. The regions of positive maximum growth among the $k=0$ modes are shaded and a few contours are plotted. Adopted from [49]

However, the important characteristic of the multicloud model is that, in the appropriate parameter regime, it exhibits a scale selective instability of wave solutions that have various key physical and dynamical features resembling observed convectively coupled equatorial waves. In Figures 17 and 18, we show linear and non-linear solutions for the multicloud equations obtained in the case of a simple flow above the equator, 
where the beta-effect is ignored. When the full (nonlinear) multicloud equations are integrated numerically, for a long enough time, with an initial condition consisting of the RCE solution plus a small random perturbation, the solution goes to a statistical steady state that exhibits the wave-like disturbances that have the same features as the their linear equivalents, including a reduced phase speed of roughly $17 \mathrm{~m} \mathrm{~s}^{-1}$, which is the speed of convectively coupled Kelvin waves, and a front-to-rear vertical tilt in wind, temperature and heating field. In particular, note that the nonlinear simulation is characterized by packets of synoptic scale waves moving at about $17 \mathrm{~m} \mathrm{~s}^{-1}$ and have a planetary scale wave envelope moving in the opposite direction at a slower speed of 5 to $6 \mathrm{~m} \mathrm{~s}^{-1}$, mimicking observed convectively coupled evolving within the MJO envelope. Consistent with "self-similarity" of tropical convection across scales, the planetary-scale envelope shares the front-to-rear tilted structure with the synoptic scale waves, though only the synoptic scale waves are linearly unstable.
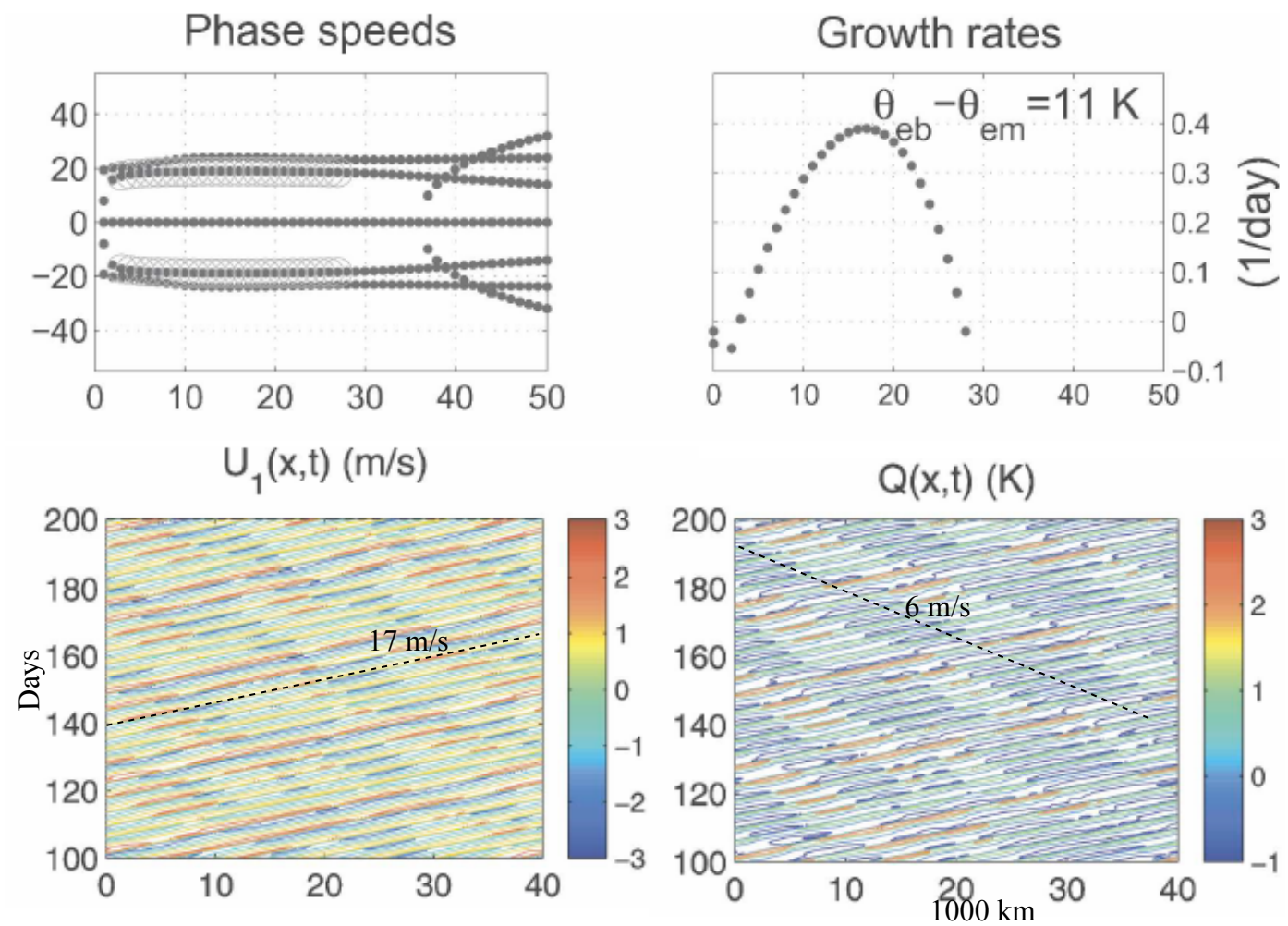

Figure 17. Top: Scale selective instability in the multicloud model with enhanced congestus closure. Bottom: (x-t) contours of the first baroclinic zonal velocity and moisture anomaly showing streaks corresponding to synoptic scale moist gravity waves moving to the right at $17 \mathrm{~m} \mathrm{~s}^{-1}$ and their planetary scale wave-envelopes moving in the opposite direction at $6 \mathrm{~m} \mathrm{~s}^{-1}$. Adopted from [50]. 

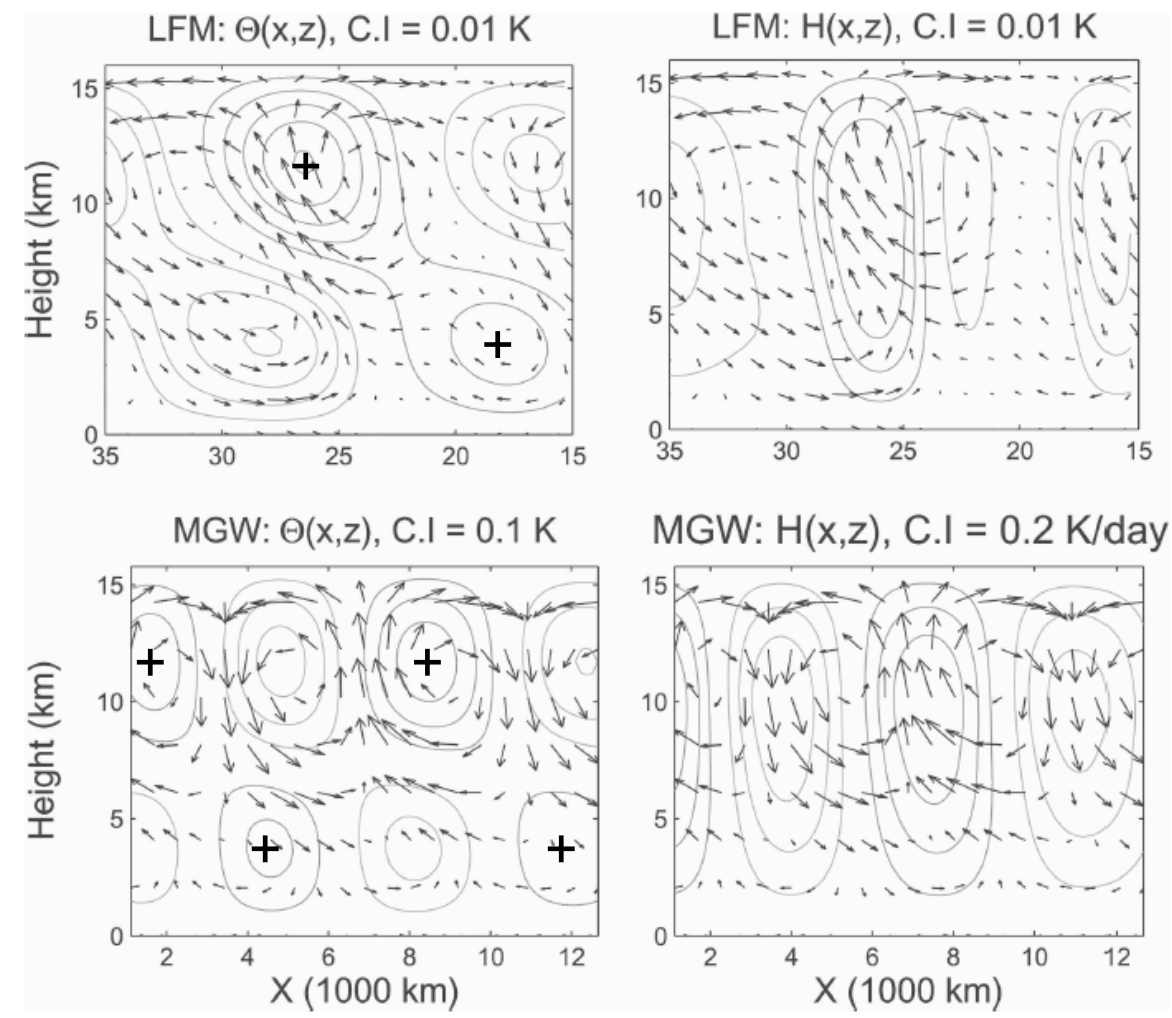

Figure 18. Filtered structure of the synoptic scale moist gravity wave (bottom) and its low-frequency planetary scale envelope (top). The left panel show potential temperature contours while the heating anomalies are contoured on the right panels with the corresponding $u-w$ velocity arrows are overlaid on top. The + signs on the temperature panels refer to positive anomalies. Adopted from [50]

Moreover, when the beta-effect is included the multicloud model exhibits instabilities corresponding to the full spectrum of convectively coupled waves seen in the observational records [47, 48], with comparable length scales and phase speeds, namely, Kelvin, westward inertio-gravity waves, and $n=0$ eastward mixed Rossby-gravity (MRG) or Yanai waves [95, 36]. Similarly, to the rotation-free case, all the simulated CCW solutions exhibit a front-to-rear tilted vertical structure as in nature. While westward MRG waves and Rossby waves are missing in the multicloud model linearized about a homogeneous RCE, they are recovered in the case of a meridional barotropic shear background, mimicking the climatological jet-stream [36]. The combination of the results shown in [95] and [36], which are summarized in Figure 19 demonstrate clearly that the multicloud model with rotation reproduces the full spectrum of convectively coupled waves that are reported in the observational literature [47, 48].

To conclude this section, we note that the results in Figure 17-19 are obtained 
with the typical synoptic scale convective time scales $\tau_{\text {conv }}=2$ hours, $\tau_{s}=3$ hours and $\tau_{c}=1$ hours, which are the estimated time scales of the deep convective, stratiform, and congestus clouds. Comparable values are also used in the stochastic multicloud model $[96,97]$.
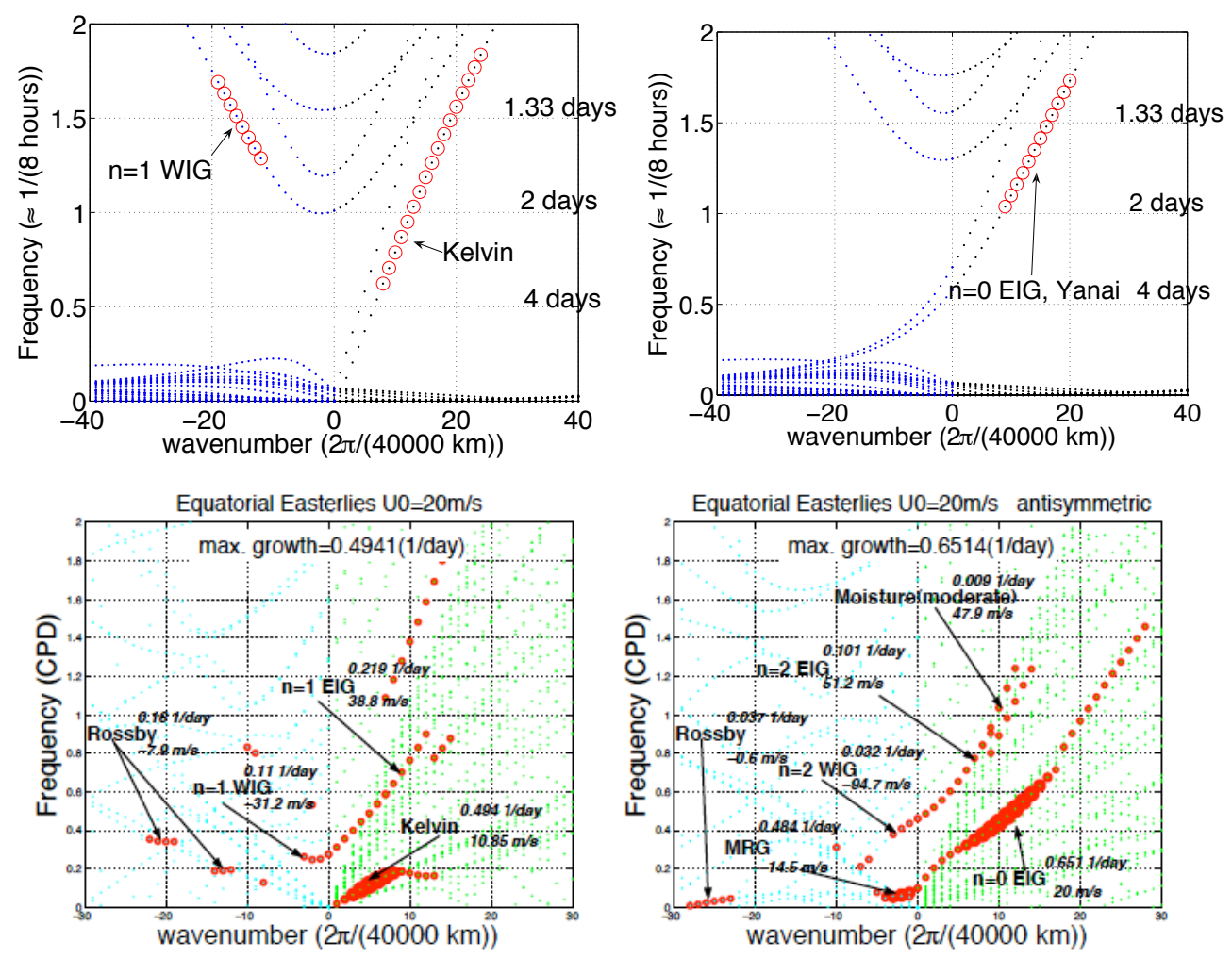

Figure 19. Dispersion diagrams for the multicloud model with rotation. Top: with a homogeneous background. Bottom: with a barotropic-meridional shear zonal wind mimicking the jet stream. Adopted from [95] and [36], respectively.

\subsection{The MJO analog wave}

By exploiting a self-similarity argument of tropical convective systems [68, 21], the multicloud model can be tuned toward a planetary and intra-seasonal scale instability of a wave that resembles the tropical intra-seasonal oscillation i.e, the MJO, as regards flow along the equator. This is accomplished in [98] by setting the convective time scales to

$$
\tau_{\text {conv }}=12 \text { hours, and } \tau_{c}=\tau_{s}=7 \text { days. }
$$

Recall that for the case of the synoptic scale waves above, we have $\tau_{\text {conv }}=2$ hours, $\tau_{c}=1$ hour and $\tau_{s}=3$ hours. The main effect of this change in parameter value is to 
shift the instability band to larger scales in both time and space. Consistently, numerical simulations with these parameter values yield a solution with the following attractive features that essentially characterize the east-west flow of the MJO above the equator, as shown in Figure 20 [98]:

A. An actual propagation speed of roughly $5 \mathrm{~m} \mathrm{~s}^{-1}$ as predicted by linear theory.

B. A wavenumber 2 structure for the low-frequency planetary-scale envelope with distinct active and inactive phases of deep convection.

C. An intermittent turbulent chaotic multiscale structure within the wave envelope involving embedded westward and eastward-propagating deep convection events.

D. Qualitative features of the low-frequency averaged planetary-scale envelope from the observational record in terms of, e.g., vertical structure of heating and westerly wind burst.
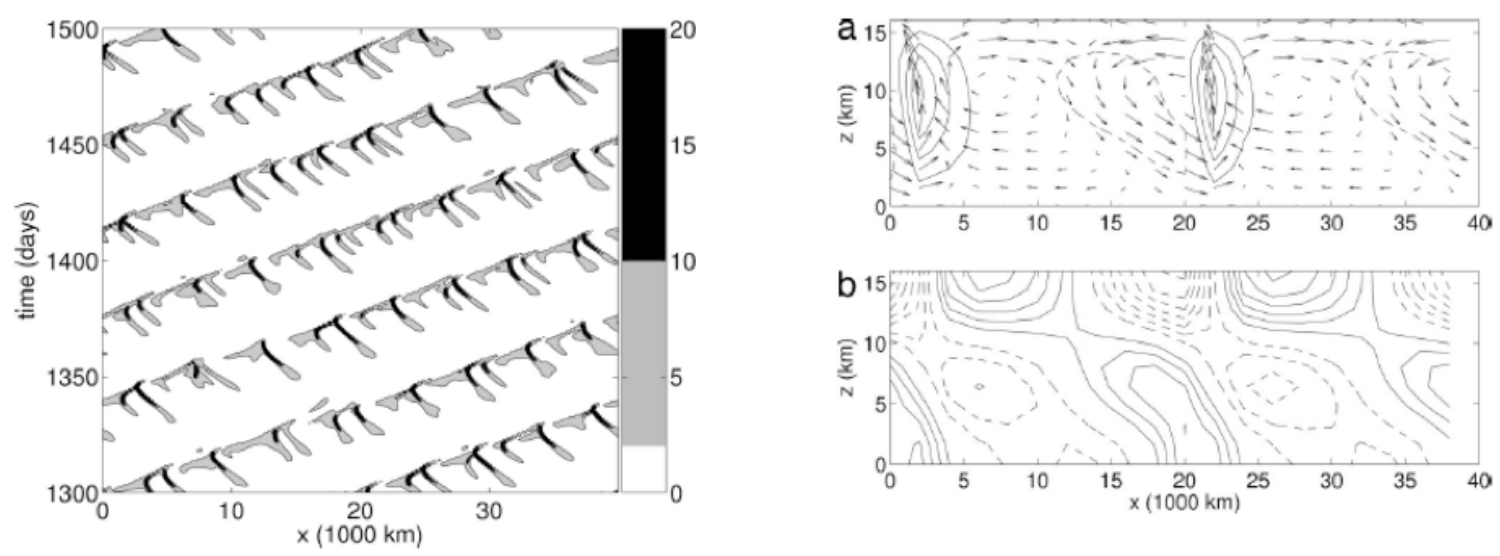

Figure 20. MJO analog wave obtained by convective time scaling for the multicloud model. Left: $\mathrm{x}$-t contours of precipitation showing slowing moving wave envelopes of mesoscale chaotic convective events that evolve within the active phase and propagate in the opposite direction. Right: vertical structure of (a) the total heating, with the uw velocity overlaid, and (b) the zonal velocity for the moving average of the planetary scale envelope. Adopted from [98].

\subsection{GCM simulation of the MJO and convectively coupled waves}

Here we show an MJO solution produced by the multicloud model when implemented in the next generation climate model of the National Center for Atmospheric Research (NCAR), namely, the High Order Modeling Environment (HOMME) dynamical core. HOMME is a highly scalable parallel code for the atmospheric general circulation based on the discretization of the primitive equations on the sphere using high order spectral elements in the horizontal and finite differences in the vertical. The interested reader is invited to look into the code development papers [99, 100] and the online documentation found on the NCAR website. Here the discussion will be limited to a brief description 
of the strategy adopted to implement the multicloud parameterization in HOMME, which provides the added condensational heating to the otherwise dry model to produce realistic MJO and convectively coupled waves solutions as reported in [20].

The first step to incorporate the multicloud model in HOMME consists in designing the proper vertical profiles for the heating field associated with the three cloud types: congestus, deep, and stratiform. It turns out, for the real atmosphere the background stratification is not uniform but nevertheless the equations of motion, linearized around such a background, lead to a generalized Stürm-Louville problem for the vertical structure functions:

$$
-\frac{d}{d p}\left(p^{2} N^{2} \frac{d \phi}{d p}\right)=\frac{1}{c^{2}} \phi,
$$

where $p$ is pressure and $N^{2}=\frac{g}{\theta} \frac{d \bar{\theta}}{d z}$ is the Brunt-Väisäilä buoyancy frequency with $g$ the acceleration due to gravity and $\bar{\theta}$ the background potential temperature. This eigenvalue problem is solved numerically using finite differences [101] to extract the first two baroclinic modes of vertical structure which correspond to the $\cos (z)$ and $\cos (2 z)$ functions used in the idealized case in (3.14) where $N^{2}$ is constant (independent of height). The two eigenfunctions, $\phi_{1}, \phi_{2}$ are plotted in Figure 21 (A) together with the associated heating profiles given by the temperature basis functions, $\psi_{j}=$ $\frac{1}{p_{B}-p_{T}} \int_{p_{T}}^{p} \phi_{j}\left(p^{\prime}\right) d p^{\prime} \mathrm{j}=1,2$, according to the hydrostatic balance equation. As in the idealized case above, while $\psi_{1}$ fixes the vertical profile for deep convective heating, the function $\psi_{2}$ is consistently used for both stratiform and congestus clouds. The heating is set to zero above roughly $200 \mathrm{hPa}$ to avoid spurious heating in the upper atmosphere.

With the heating rates $H_{d}, H_{s}, H_{c}$ parameterized as in the idealized case presented above and the vertical average moisture equation rederived according to the new basis functions $\phi_{j}, \psi_{j}$ (instead of the cosines and sines), the HOMME full primitive equations are forced by the heating $H(x, y, p)=H_{d}(x, y) \psi_{1}(p)+\left(H_{c}(x, y)-H_{s}(x, y)\right) \psi_{2}(p)$. The resulting coupled model is solved on an aquaplanet with a uniform surface evaporation and standard value of radiative cooling $1 \mathrm{~K} /$ day. The moisture and temperature are initialized to a tropical mean profile which is also used to fix the Brunt-Väisäilä frequency profile in the Stürm-Liouville problem which is solved once for all at the beginning of the simulation.

In the appropriate parameter regime (see [20]), the coupled HOMME-multicloud model yields a solution consisting of two MJO-like waves that move eastward at roughly 5 $\mathrm{m} \mathrm{s}^{-1}$ and interact together in the same fashion as seen in observation. This is illustrated by (B) the x-t contours of the zonal velocity and deep convection, (C) the zonal structure of the MJO filtered zonal velocity, (D) the filtered vorticity, and (E) the vertical structure of the filtered zonal velocity shown in Figure 21. Note in particular the MJO solution has the same tilted structure, intraseasonal period, and imbedded mesoscale turbulent fluctuations in the deep convective heating as in the case of the MJO analog of Figure 20 and in addition, the vorticity field is characterized by a quadrupole vortex surrounding the westerly-wind burst. The interested reader is referred to the original paper [20] for more discussion of this solution and others. In particular, the whole spectrum of 
synoptic scale convectively coupled waves is reproduced in the appropriate parameter regime.

(A)

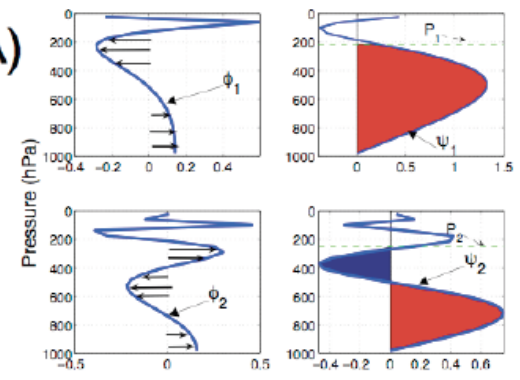

(B)
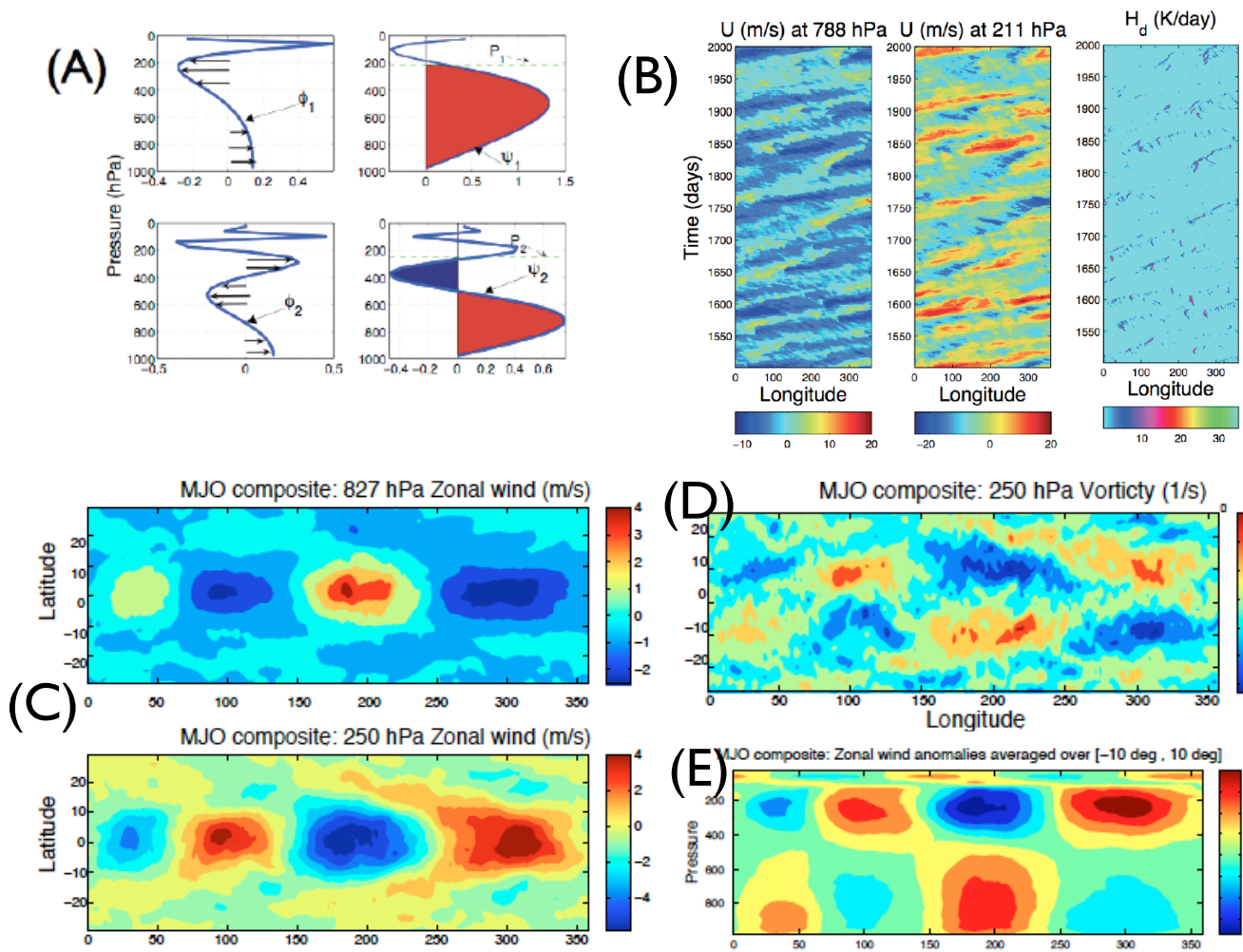
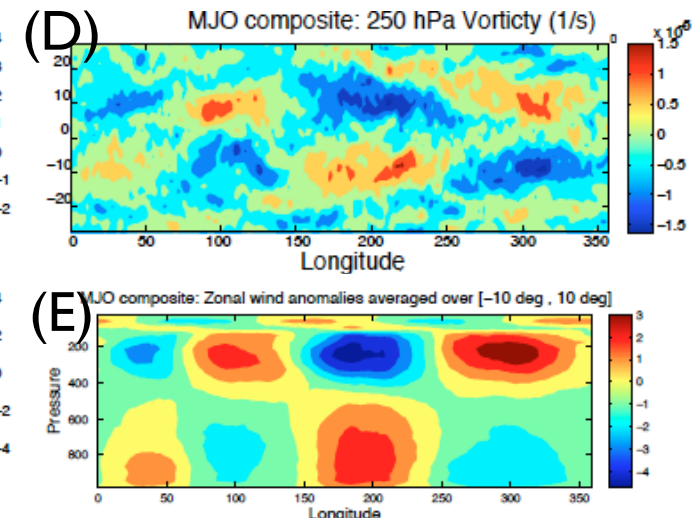

Figure 21. Eigenmode and heating profiles used to implement the multicloud model in HOMME: (A) and the resulting MJO solution: (B) the $\mathrm{x}$-t contours of the zonal velocity and deep convection, $(\mathrm{C})$ the zonal structure of the MJO filtered zonal velocity and (D) filtered vorticity, and (E) vertical structure of the filtered zonal velocity. Adopted from [20].

\subsection{Convective momentum transport and multiscale waves within the MJO}

Convective clouds not only release heat and moisture into the troposphere but they also deposit momentum due to turbulent eddies associated with the convecting parcels. This phenomenon known as convective momentum transport (CMT), can also be applied to turbulent fluxes associated with mesoscale and synoptic scale convective organized systems when regarded from larger scales. The small eddies associated with parcel lifting essentially result in large-scale momentum damping in an effect known as cumulus friction, due to their chaotic nature just like the usual fluid dynamics turbulence; in contrast, there is enough observational, numerical, and theoretical evidence that the CMT associated with mesoscale synoptic scale organized convective systems yield very 
coherent momentum fluxes that can accelerate or decelerate the ambient flow depending on the circumstances [102, 103, 104, 105, 106, 107, 73, 108, 109].

The mesoscale and synoptic scale waves in particular yield a non-zero vertical momentum flux thanks to their backward tilted vertical structure [64]. Consider for example the zonal momentum equation and assume that the flow is divided into a mean and fluctuation parts: $u=\bar{u}+u^{\prime}$. Assume that the fluctuation part has a vertical structure on the form $u^{\prime}=-f(x) \cos (z)-g(x) \cos (2 z)$ where $f(x)$ and $g(x)$ vanish quickly enough at infinity or are periodic functions. Let $w^{\prime}=f^{\prime}(x) \sin (z)+\frac{1}{2} g^{\prime}(x) \sin (2 z)$ be the vertical velocity associated with $u^{\prime}$. It is easy to see that the associated turbulent flux is given by

$$
-\left\langle u^{\prime} w^{\prime}\right\rangle_{z}=-\frac{3}{4} \overline{f^{\prime} g}[\cos (z)-\cos (3 z)] .
$$

Here the overbar is a zonal average. Therefore, the CMT resulting from the combination of deep convective and stratiform (and/or congestus) clouds forces the first and third baroclinic of the large-scale zonal wind $\bar{u}$ but not the second mode. To ensure positive heating we require $f^{\prime}(x) \geq 0$ and $g^{\prime}(x) \leq 0$, in the case of stratiform deep combination. If in addition, we assume that $g(x)=-2 \alpha f\left(x+x_{0}\right), 0<\alpha<1$, i.e, stratiform heating is proportional to and lagging behind deep convection, then the CMT flux is zero if $x_{0}=0$ or $\alpha=0$. Thus the statement that a vertical tilt, in the zonal and vertical winds, is necessary to achieve a non-zero mesoscale and/or synoptic scale CMT [64].

For small enough $x_{0}$ we have $\overline{f^{\prime}(x) f\left(x+x_{0}\right)} \approx \overline{f^{\prime}(x) f(x)}+x_{0} \overline{\left[f^{\prime}(x)\right]^{2}}=x_{0} \overline{\left[f^{\prime}(x)\right]^{2}}$, i.e, the convective momentum flux satisfies

$$
-\left\langle u^{\prime} w^{\prime}\right\rangle_{z} \approx \frac{3}{2} \alpha x_{0} \overline{\left[f^{\prime}(x)\right]^{2}}[\cos (z)-\cos (3 z)]
$$

The last expression has the same sign as $x_{0}$ near the surface, i.e, positive for westward tilted waves $\left(x_{0}>0\right)$, and negative for eastward tilted waves $\left(x_{0}<0\right)$. The full vertical structure of the CMT flux is plotted in Figure $22(\mathrm{~A})$ for both $x_{0}>0$ and $x_{0}<0$. According to Figure $22(\mathrm{~A})$, westward tilted waves accelerate large-scale westerly winds in the lower troposphere and accelerate easterlies in the upper troposphere while eastward tilted waves do the opposite. Accordingly, observations revealed that when there is organized mesoscale squall line convection, CMT is positively correlated, thus accelerates, the background shear and when convection is scattered, CMT and the background shear are negatively correlated. See Panels (C) and (D) of Figure 22.

Based on the WTG asymptotic approximation, Majda and Stechmann [108] proposed a stochastic jump Markov model to account for CMT due unresolved mesoscale systems in large scale climate models. According to the observation above and the observational evidence reported in Figure 22 (panels (C) and (D)), they constructed a three state Markov chain. When deep convection is active and the vertical shear is strong, a signature of large-scale organized convection, a state of positive (acceleration) CMT is assigned with high probability, when deep convection co-exists with a weak or no vertical shear background, the Markov process transits to a cumulus friction state with high probability and finally when deep convection is absent it goes to a damping state. It 
demonstrated in [108] that the stochastic CMT model accelerate the background shear which is assumed to generate mesoscale convective system that are tilted in backward direction, just like in the observations reported in Panels (C) and (D) of Figure 22. A mock-test using a single ODE of a background shear forced by the stochastic CMT model is reported in Figure 22 (B) [108]. It shows a consistent acceleration of the low-level shear. Moreover, Majda and Stechmann [108], demonstrated that when the stochastic CMT model is coupled to the multicloud model both the variability and overall organization of convectively coupled gravity waves is enhanced.

Khouider et al. [109] used a similar but deterministic CMT model, essentially mimicking the meanfield limiting dynamics of the [108] model, in a case study related to the multicloud model experiment reported in Figure 18 above. The demonstrated that when the mesoscale CMT model is included in the multicloud model, the planetary scale envelope wave in Figure 18 becomes much stronger; it amplifies by about ten orders of magnitude making it as strong as the embedded synoptic scale waves, in addition to decreasing the wavenumber of both the planetary and the synoptic scale waves, from 2 to 1 and from 6 to 5, respectively. This is consistent with the finding of [108] and with Figure 22 according to which CMT enhances low low-level shear and in particular the mean wind at around $4 \mathrm{~km}(700-800 \mathrm{hPa})$. This would enhance the low-level moisture convergence in the convection center of the large scale wave, i.e, the fuel for deep convection.

The multiscale organization of synoptic and mesoscale convectively coupled waves is considered in [74], based on the multicloud model. Building on the earlier work of Majda and Stechmann [73] and Han and Khouider [36], they studied the behavior of convectively coupled tropical waves and tropical squall lines in an MJO background, represented by both zonal wind shear and vertical moisture profiles, representative of the various phases of the MJO. Based on the linear stability diagrams of the multicloud model with rotation, it is demonstrated in [74] that convectively coupled Kelvin waves have the largest growth rates in the eastern part of the MJO corresponding to easterlies in the lower troposphere overlaid by upper level westerlies and a moist below and dry above troposphere while westward inertio-gravity waves (aka two-day waves) are dominantly unstable in the westerly wind burst region on the western part of the MJO which is characterized by low-level westerlies beneath upper level easterlies and a moist upper troposphere and a relatively dry lower troposphere. Squall lines on the other hand dominate the convective core and propagate in the direction of the low-level wind towards and from both sides of the convection center. In [74], the plausible implications of such embedded multiscale organization of synoptic and mesoscale waves on the amplification and the dynamics of the MJO envelope were suggested, namely, through the CMT mechanism. However, observational evidence and/or detailed numerical simulations confirming this behavior remain to be developed. 

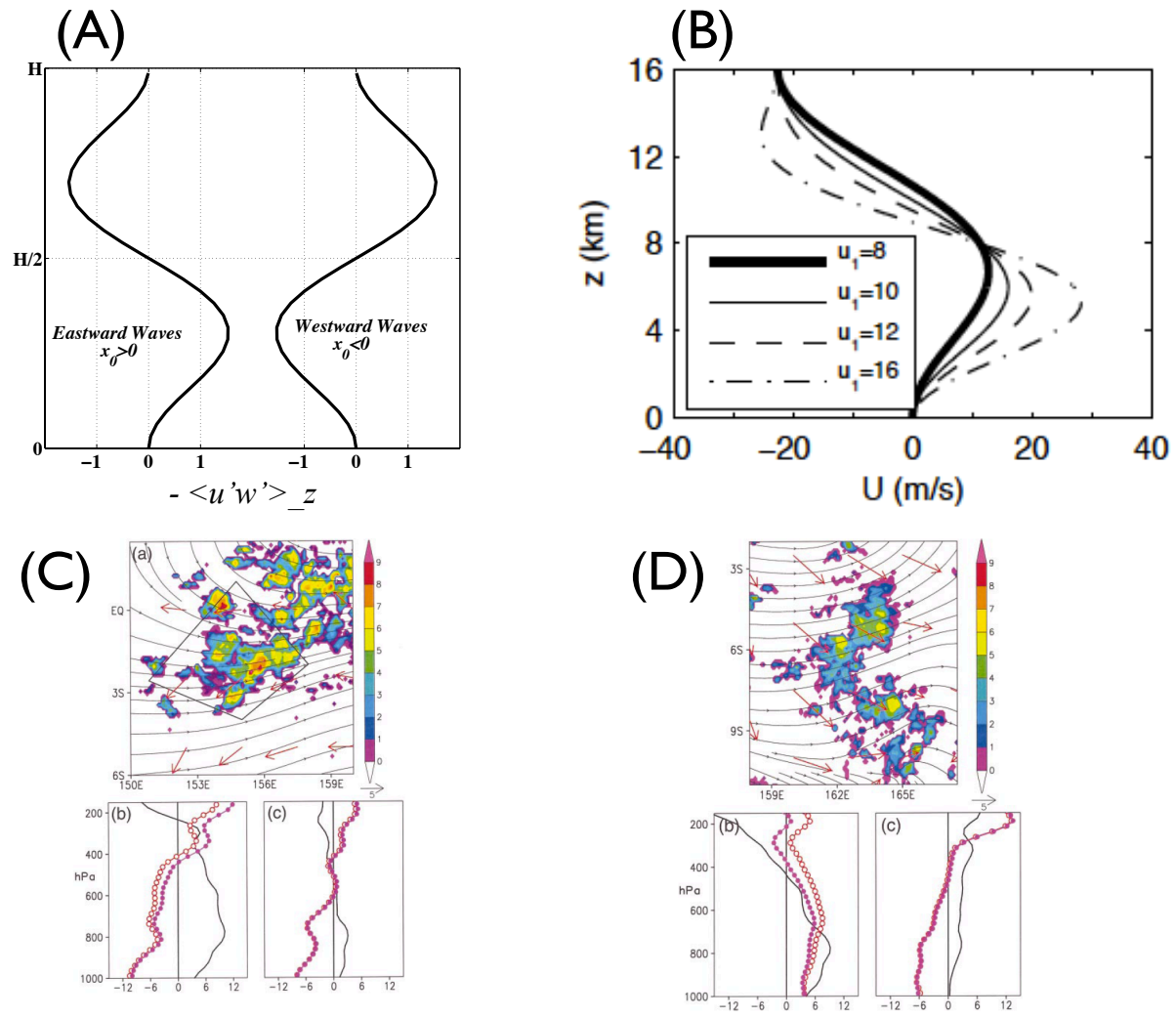

Figure 22. (A) Vertical structure of CMT flux associated with eastward and westward waves. (B) Acceleration of a large-scale mean shear flow by a stochastic CMT parameterization. Adopted from [108]. (C) and (D) observation of CMT fluxes due respectively to scattered and organized mesoscale convection and the associated mean flow: (a) radar reflectivity (rain), (b) vertical structure of CMT flux $-\left\langle u^{\prime} w^{\prime}\right\rangle_{a}$ (circles) and mean zonal wind $\bar{u}$, and (c) same as (b) but for $\left\langle v^{\prime} w^{\prime}\right\rangle$ and $\bar{v}$. Adopted from [107].

\subsection{A multiscale model for the MJO: the muscle}

As described above the MJO is a planetary scale envelope of complex multiscale convective waves that begins as a standing wave over the Indian Ocean warm pool and propagates at a slow phase speed of roughly $5 \mathrm{~m} \mathrm{~s}^{-1}$, eastward, toward the Western Pacific Ocean. Here we present a multiscale model that mimics across-scale interaction between the MJO and the embedded synoptic scale superclusters. The synoptic scale waves, evolving within the MJO envelope, provide the mean heating and "turbulent" fluxes of momentum and temperature that are necessary to drive the MJO-skeleton. In some sense the model presented here constitute the muscle that drives the MJO dynamics. This model was first proposed by Majda and Biello [64] and later improved and refined by the same authors in [65]. It is based on the multiple scale IPESD model discussed in section 4.1 . 
Majda and Klein [59] derived the IPESD equations already presented in (4.2), (4.3) from the equatorial beta-plane primitive equations. The derivation sketched below follows the shallow water derivation from [59]; see [68] for an important generalization and simplified derivation in the present setting. This derivation begins by assuming a multiple scales asymptotic expansion with the form $u=\bar{u}(\epsilon x, y, z, t)+$ $\epsilon u^{\prime}(\epsilon x, x, y, z, t)+O(\epsilon)$ for zonal velocity, pressure, and temperature with the form $v=v^{\prime}(\epsilon x, x, y, z, t)+\epsilon \bar{v}(\epsilon x, y, z, t)$ for the vertical and meridional velocity components, where $\epsilon>0$ is a small parameter that represents the Froude number and estimated to be around $1 / 6$. Here $x, y, z$ are the zonal, meridional, and vertical coordinate variables that evolve on the synoptic scale while the time scale is an intraseasonal time which has units of roughly 3.5 days, roughly four times the synoptic time scale. The end result consists of two linear PDE systems, one for the synoptic scale fluctuations, $u^{\prime}, v^{\prime}$ etc., and one for the planetary scale variables, $\bar{u}, \bar{v}$. Here the overbar is a zonal average over a synoptic scale periodic domain. On the synoptic scales, we have the synoptic-scale equatorial weak temperature gradient equations (SEWTG) [59, 64, 65]

$$
\begin{aligned}
& -y v^{\prime}+p_{x}^{\prime}=0, y u^{\prime}+p_{y}^{\prime}=0, \quad w^{\prime}=S_{\theta}^{\prime} \\
& p_{z}^{\prime}=\theta^{\prime}, u_{x}^{\prime}+v_{y}^{\prime}+w_{z}^{\prime}=0 .
\end{aligned}
$$

On the planetary scale, we have the "quasi-linear" equatorial long-wave equations (QLELWE) [59, 64, 65]

$$
\begin{aligned}
& \bar{u}_{t}-y \bar{v}+\bar{p}_{X}=F_{u}-d \bar{u}, y \bar{u}+\bar{p}_{y}=0, \\
& \bar{\theta}_{t}+\bar{w}=F_{\theta}-d \overline{+} \bar{S}_{\theta} \\
& \bar{p}_{z}=\bar{\theta}, \quad \bar{u}_{x}+\bar{v}_{y}+\bar{w}_{z}=0 .
\end{aligned}
$$

Here $u, v, w$ are respectively the zonal, meridional, and vertical velocity components, $\theta$ is the potential temperature, $p$ is pressure, and $X=\epsilon x$ is the planetary scale zonal variable. The synoptic and planetary scale heating terms $S_{\theta}^{\prime}$ and $\bar{S}_{\theta}$ satisfy $S_{\theta}=$ $S_{\theta}^{\prime}(\epsilon x, x, y, z, t)+\epsilon \bar{S}_{\theta}(\epsilon x, y, z, t)$ and $\bar{S}_{\theta}^{\prime}=0$ by design. The terms $F_{u}=-\overline{\left(u^{\prime} v^{\prime}\right)_{y}}-\overline{\left(u^{\prime} w^{\prime}\right)_{z}}$ and $F_{\theta}=\left(\theta^{\prime} v^{\prime}\right)_{y}-\overline{\left(\theta^{\prime} w^{\prime}\right)_{z}}$ are the turbulent fluxes that account for the effects of synoptic scales on the planetary scale, thus the quasi-linear nature of the coupled system. Given the specific synoptic scale and planetary scale (or mean) heating rates, Majda and Biello $[64,65]$ solved the linear system in (5.7) exactly and computed these turbulent fluxes. The later are then used to force the planetary scale equations.

Inspired by previous simple models $[63,110,14]$ of large scale tropical circulation and more recent observations [111, 48, etc] Majda and Biello [64, 65] assumed a prescribed heating with the form $S_{\theta}=F(X-s t)\left[Q^{\prime}(X, x, y, z, t)+\epsilon \bar{Q}(X, y, z, t)\right]$ where $F(X-s t)=\cos \left[\pi(X-s t) / 2 L_{F}\right]^{+}$describes the envelope of convection that moves at a prescribed speed $s=5 \mathrm{~m} \mathrm{~s}^{-1}$ where $L_{F}=5000 \mathrm{~km}$ is the length scale of the envelope. Both the mean and synoptic scale fluctuations consist of a deep convective part which projects onto the first baroclinic $\sin (z)$ mode plus a stratiform and/or congestus part which projects onto the second baroclinic $\sin (2 z)$ mode. They take the form $\bar{Q}(X, y, z, t)=H(y)[\sin (2 z)+\bar{\alpha} \sin (2 z)]$ and $Q^{\prime}(X, x, y, z, t)=$ 
$H(y)\left\{f[x-\phi(t)] \sin (z)+\alpha f\left[x+\phi_{0}-\phi(t)\right] \sin (2 z)\right\}$ where $\phi(t)$ is the phase of the propagating synoptic scale wave trains and $\phi_{0}$ is an assumed time lag between the first and second baroclinic modes. Similarly, the coefficients $\alpha$ and $\bar{\alpha}$ take the form $\alpha=8(X-s t) / 3 L_{F}$ and $\bar{\alpha}=8(X-s t) / 3 L_{F}$. In this fashion, the mean heating is bottom heavy in front of the envelope and top heavy in the back, mimicking the congestus cloud decks that are observed to lead the MJO and the trailing stratiform anvils, respectively. At the same time, the synoptic scale heating is composed by wave trains of congestus systems in front of the envelopes and trailing westward tilted super-clusters in the back. The function $H(y)=e^{-\left(y-y_{0}\right)^{2} / 2}$ is a simple Gaussian function that confines the heating to the tropics. We recall that the mean heating feeds directly into the planetary scale equations while the synoptic scale heating is used to produce the turbulent fluxes $F_{u}$ and $F_{\theta}$. The wave train character of the synoptic scale heating is given by $f$ which is a periodic function of the synoptic scale variable $x$. It takes the form of either a Gaussian or a full cosine and integrates to zero over the synoptic scale period. Further details can be found in $[64,65]$.

The left and right top panels of Figure 23 displays the assumed synoptic scale packets of westward tilted superclusters and congestus waves, respectively. The bottom two panels show the horizontal and vertical structure of the resulting MJO wave. The horizontal velocity arrows and pressure contours on the left display, in the upper troposphere, a pair of anticyclones in the MJO center preceding a much larger pair of cyclones in the back, forming a quadrupole vortex. In the lower troposphere easterly winds precede westerlies at the equator. The vertical structure of the zonal wind at the equator on the bottom left panels is characterized by a strong westward tilt. These are among the main dynamical features which characterize the MJO in observations as discussed in the previous sections.

When the effects of the mean heating and westward tilted and congestus waves trains were considered separately, Biello and Majda [65] noted that the MJO tilt results from the tilted mean heating while the quadrupole vortex is due to the turbulent momentum and thermal fluxes of the synoptic scale wave trains.

Historically, the Matsuno-Gill model [63] consists of a simple linear shallow water system (the equivalent of the first baroclinic mode alone) forced by a stationary heat source, which also is confined to the tropics. The result is a complex circulation pattern that essentially consist of a (half of) Kelvin wave in front of the heating center and a (half of) Rossby wave in the back. As such it lacks the two anti-cyclonic gyres in front and has no-vertical tilt. However, because of the first baroclinic structure, the meridionally averaged flow resembles a two-cell Walker circulation while the zonally averaged flow looks like a double Hadley cell. Chao [110] included a time variation to the Gill model and made the heat source slowly move eastward at $5 \mathrm{~m} \mathrm{~s}^{-1}$. The structure remained the same as in the original model but it now moves eastward as an MJO. The systematic derivation, the inclusion of the synoptic scale turbulent fluxes from modulated synoptic scale convectively coupled waves and the tilted mean heating are the key physical features of the Majda-Biello model that resulted in a more realistic 
(i)

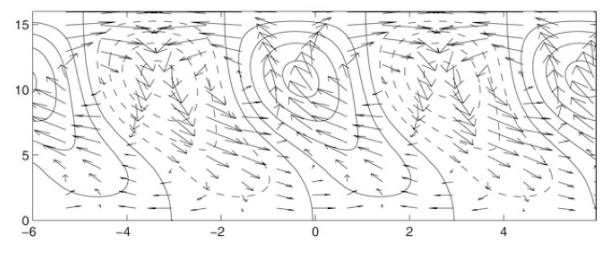

(iii)
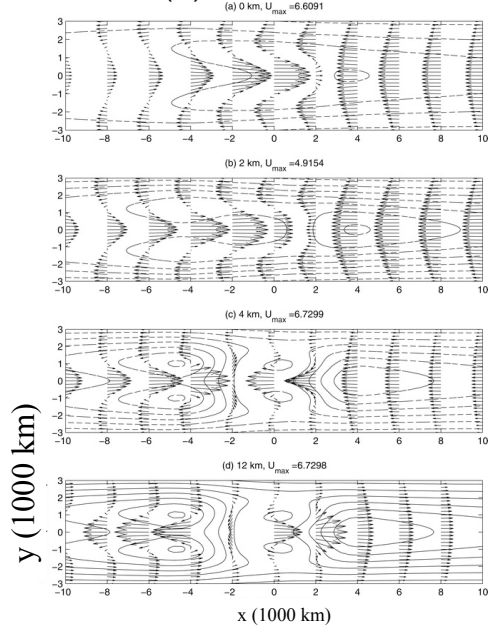

(ii)

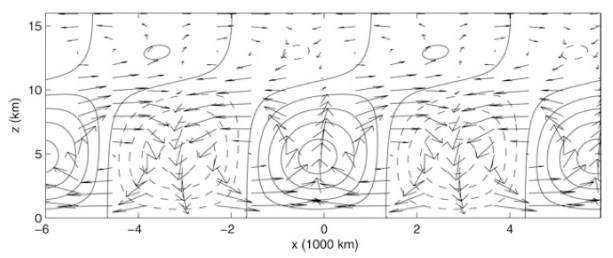

(iv)
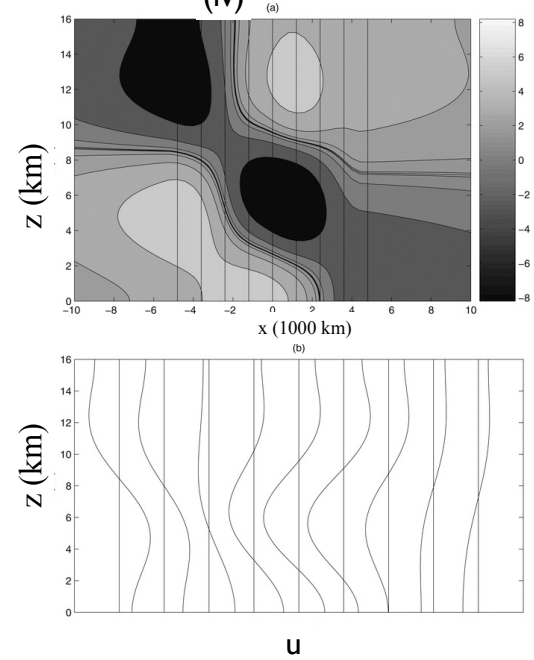

Figure 23. Vertical structure of the westward tilted (i) and congestus (ii) synoptic scale wave packets used to the force the planetary scale QLELW equations 5.8. The horizontal (iii) and vertical structure (iv) of the zonal wind for the MJO solution for the tilted mean heating and westward tilted and congestus synoptic scale wave trains. See text for details. Adopted from [65].

MJO solution. Note that the quadrupole vortices in the MJO can be explained through other simple mechanisms as described earlier in section 4.4 and Figure 13.

\section{Tropical-extratropical interactions}

Most of the energy from the sun which is absorbed by the sea surface within the tropical belt is directly transported by convection and transferred to large scale convectively coupled equatorial waves. Linear and non-linear dynamical interaction between equatorially trapped waves and barotropic planetary scale Rossby waves are believed to be the main engines through which energy is transferred from the tropics to midlatitudes and vice-versa. The background meridional shear, i.e, the jet stream, can force equatorial waves in various ways so as to modify their meridional structure and propagation speed and serve as a medium through which equatorial waves can exchange energy with each other. Tropical waves can resonate with the diurnal cycle through 
convection and midlatitude Rossby waves can act as a trigger for MJO initiation. Here we will review various papers that addressed some of these issues in simple settings. The interested reader is referred to the papers mentioned here for further details.

\subsection{Nonlinear resonance of longwave barotropic and baroclinic Rossby waves}

Reference [23] contains the derivation of new KdV-like equations for the nonlinear resonant interaction between barotropic and baroclinic Rossby waves. Consider the barotropic-baroclinic equations (3.3) of section 3.1.1 with the barotropic flow written in vorticity stream function form and introduce the zonal longwave scaling [23]:

$$
\begin{aligned}
& \bar{\psi}=\bar{\psi}(\delta x, y, \delta t), \quad \theta_{1}=\theta_{1}(\delta x, y, \delta t) \\
& u_{1}=u_{1}(\delta x, y, \delta t), \quad v_{1}=\delta v_{1}(\delta x, y, \delta t) .
\end{aligned}
$$

Here $\delta=L_{E} / L_{P}=T_{E} / T_{P}$ with $L_{P} \approx 5000 \mathrm{~km}$ and $T_{P}=O(3$ days $)$ are the zonal and temporal scales on which planetary scale Rossby wave coupling is assumed to occur. In terms of these long zonal and slow time variables, the coupled barotropic-baroclinic system (3.3) becomes [23, 29]

$$
\begin{aligned}
& \frac{\bar{D} u_{1}}{D t}-\mathbf{v}_{1} \cdot \bar{\psi}_{y}-y v_{1}-\theta_{x}=0 \\
& \delta^{2}\left(\frac{\bar{D} v_{1}}{D t}+\mathbf{v}_{1} \cdot \nabla \bar{\psi}_{x}\right)+y u_{1}-\theta_{y}=0 \\
& \frac{\bar{D} \theta_{1}}{D t}+\nabla \cdot \mathbf{v}_{1}=0 \\
& \frac{\bar{D} \bar{\psi}_{y y}}{D t}+\bar{\psi}_{x}-\nabla \cdot\left(u_{1} \mathbf{v}_{1}\right)_{y}+\delta^{2}\left[\frac{\bar{D} \psi_{x x}}{D t}+\nabla \cdot\left(v_{1} \mathbf{v}_{1}\right)_{x}\right]=0 .
\end{aligned}
$$

When the $O\left(\delta^{2}\right)$ and non-linear terms are dropped in (6.2), we obtain the linear longwave versions of the baroclinic and barotropic shallow-water equations in (3.6) and (3.5). These decoupled equations support longwave baroclinic Rossby waves with a dispersion relation $c_{n}=\frac{-1}{2 n+1}+O(\delta)$ and eigenmode structure

$$
\begin{aligned}
& u_{b c}=\frac{A_{n}\left(x-c_{n} t\right)}{\sqrt{2}}\left[D_{n-1}(y)-\frac{D_{n+1}(y)}{n+1}\right] \\
& v_{b c}=-\frac{2}{2 n+1} \frac{\partial A_{n}\left(x-c_{n} t\right)}{\partial x} D_{n}(y) \\
& \theta_{b c}=\frac{A_{n}\left(x-c_{n} t\right)}{\sqrt{2}}\left[D_{n-1}(y)+\frac{D_{n+1}(y)}{n+1}\right]
\end{aligned}
$$

and longwave barotropic Rossby waves with $\bar{c}_{l}=-\frac{1}{l^{2}}$ and structure given by

$$
\bar{\psi}_{R}=-B\left(x-\bar{c}_{l} t\right) \sin (l y) .
$$

Here, the $D_{n}$ 's are parabolic cylinder functions, $l$ is the meridional wavenumber, and $x$ and $t$ are the long zonal scale and slow time variables measured in terms of the planetary scales $L_{P}$ and $T_{P}$, respectively. 
Resonant interactions between the longwave barotropic and baroclinic waves occur when $\bar{c}_{l}=c_{m}$ [23] which requires $l=\sqrt{2 n+1}$ and yields a meridional wavelength $L_{*}=(2 \pi / \sqrt{2 n+1}) L_{E}$ for the barotropic Rossby wave where this resonant interaction takes place. Thus equatorial Rossby waves of meridional index $n=1,2,3$ are expected to resonate with barotropic Rossby waves on meridional wavelengths of about $L_{*}=5440,4200,3600 \mathrm{~km}$, respectively [23, 29].

To look at the weak nonlinear interactions between these two waves, following [64] we assume the multiple time scale ansatz

$$
u=\epsilon u_{b c}(x, y, t, \epsilon t)+\epsilon^{2} u^{(2)}, \quad \bar{\psi}=\epsilon \psi_{R}(x, y, t, \epsilon t)+\epsilon^{2} \bar{\psi}^{(2)},
$$

for the rescaled barotropic-baroclinic system (6.2), where we introduced an even longer time scale $\tau=\epsilon t$ so that the wave amplitudes $A$ and $B$ are allowed to vary on a slower time scale. A similar ansatz is used for $v$ and $\theta$.

Systematic asymptotics with $\epsilon=\delta^{2}$ then leads to a $\mathrm{KdV}$-like system of equations for the amplitudes $A$ and $B$ of the longwave baroclinic and barotropic Rossby waves $[23,29]$. After some careful rescaling, it takes the simple form

$$
\begin{aligned}
& A_{\tau}-D A_{x x x}+(B A)_{x}=0, \\
& B_{\tau}-B_{x x x}+A A_{x}=0,
\end{aligned}
$$

where $D$ is a parameter depending only on the meridional index $n$ (or equivalently on l). For $m=1,2,3$, we have $D=0.889,0.960,0.980$, respectively [23].

The system in (6.4) has very interesting mathematical properties including energy conservation and wave dispersion [23]. While the conservation of $E=\frac{1}{2} \int A^{2}+B^{2} d x$ is consistent with the energy conservation for the barotropic-baroclinic system (3.3), other features are better illustrated by the introduction of the zonal means $\bar{A}, \bar{B}$ :

$$
A(x, \tau)=\bar{A}(\tau)+A^{\prime}(x, \tau), B(x, \tau)=\bar{B}(\tau)+B^{\prime}(x, \tau)
$$

where the $A^{\prime}, B^{\prime}$ are the wave fluctuations with zero zonal average and $\bar{A}, \bar{B}$ can be regarded as the strength of the background vertical and meridional zonal wind shear. Note that the associated background shears have the same meridional structure as the wave fluctuations. Similar equations for the case of arbitrary meridional and vertical zonal wind shears are derived and analyzed in [29]; there, the more general case with both symmetric $(\sin (l y))$ and anti-symmetric $(\cos (l y))$ barotropic Rossby waves is considered.

As stated in [23], we have

$$
\frac{1}{2} \frac{d}{d \tau} B^{\prime 2} d x=-\frac{1}{2} \frac{d}{d \tau} \int A^{\prime 2} d x=\frac{1}{2} \int\left(A^{\prime}\right)^{2} B_{x}^{\prime}+\bar{A}\left(A^{\prime} B_{x}^{\prime}-B^{\prime} A_{x}\right) d x .
$$

This shows in addition that the wave energy is conserved and the energy exchange between the baroclinic and the barotropic waves occurs through the specific terms on the right hand side of this expression and that the mean baroclinic (vertical) shear $\bar{A}$ contributes to this exchange.

The linearization of (6.4) about a constant state $\bar{A}, \bar{B}$ (independent of $\tau$ ) yields the following explicit formula for the phase speed [23].

$$
c_{ \pm}(k)=\frac{\bar{B}+k^{2}(1+D)}{2} \pm \frac{\left\{\left[k^{2}(1-D)-\bar{B}\right]^{2}+4 \bar{A}^{2}\right\}}{2} .
$$


This corresponds to stable dispersive waves propagating in both, zonal directions, fairly rapidly with respect to the slow time $\tau$. Accordingly, they induce significant corrections to the background phase speed $c_{m}$ of the resonating Rossby waves.

The system in (6.4) is extensively studied in [23] and [29] using both linear and non-linear simulations and the physical mechanism of these Rossby wave interactions is illustrated. It is shown for instance that it takes typically about 15 to 20 days for the energy to be transfered from one wave to the other. A typical numerical solution for (6.4), taken from [23], that describes the energy transfer from the barotropic wave to the baroclinic wave is displayed in Figure 24. The solution in Figure 24 has the interesting feature of generating a "westerly wind burst" from this extratropical interaction which is a characteristic features of the Madden-Julian oscillation.

Soliton-like solutions for the amplitude equations (6.4) were derived in [29] and validated numerically, using the full system (3.3), in [43]. They take the form

$$
A(x, t)=\bar{A}+\alpha \operatorname{sech}^{2}\left(k_{1}\left(x-c_{s} t\right)\right) ; B(x, t)=\bar{B}-\beta \operatorname{sech}^{2}\left(k_{1}\left(x-c_{s} t\right)\right)
$$

where $c_{s}$ is the total wave propagation of the solitary wave. In Figure 25, we display the solution of (3.3) when the initial condition coincides with this solitary wave. The idea here is to verify whether this solitary wave is close to an actual solution of (3.3) and how long it takes before it breaks down. According to the longwave approximation, any such solution will break down when small scale features start to form, i.e, as energy cascades down to small scales. As we can see from Figure 24, the soliton wave remains close to the solution of (3.3) up to 2 days. After this period, small scale features start to form [43] and energy starts to flow back and forth between the barotropic and baroclinic modes (Figure 24). A strong dissipation mechanism maybe required in order to maintain the longwave soliton-like solution. The effect of boundary layer dissipation on the barotropic-baroclinic Rossby wave interaction is considered in [28].

\subsection{Barotropic response to a slowly moving Kelvin wave}

We now consider the system in (3.3) when the baroclinic flow is artificially frozen to a simple Kelvin wave $u=\alpha_{0} e^{-y^{2} / 2} \cos \left(k_{0}\left(x-c_{0} t\right)\right)$ and is used to force the barotropic equations. Here $\alpha_{0}, k_{0}, c_{0}$ are free parameters that set the strength, the wavenumber, and phase speed of the Kelvin wave, respectively. We are interested in the case when both $k_{0}$ and $c_{0}$ are small, a slowly moving synoptic to planetary scale wave. The ubiquity of slowly moving moisture Kelvin-like waves in the tropical atmosphere makes this model very reasonable. The scientific question is how such "freely" moving waves influence the rest of the atmosphere and in particular the mid-latitude flow patterns that govern our day-to-day weather and climate. Because the barotropic waves are not trapped in the

vicinity of the equator, the barotropic flow constitutes a good candidate for this task. The feedback on the Kelvin wave, from the barotropic flow it generates, is neglected for simplicity. This model is proposed and studied in [112]. 
(a) Sostal Ancinuss, $\mathrm{t}=0$

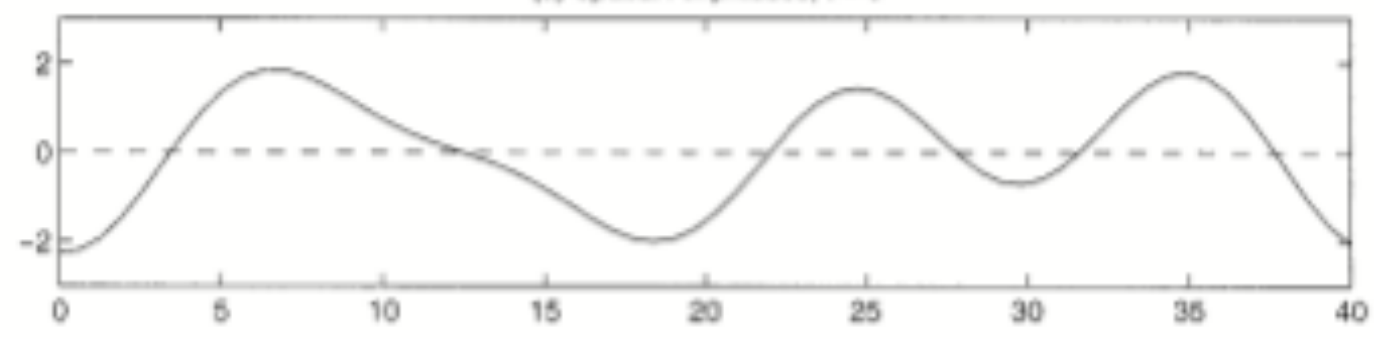

(b) Veibeligy at bentem of trepesphere

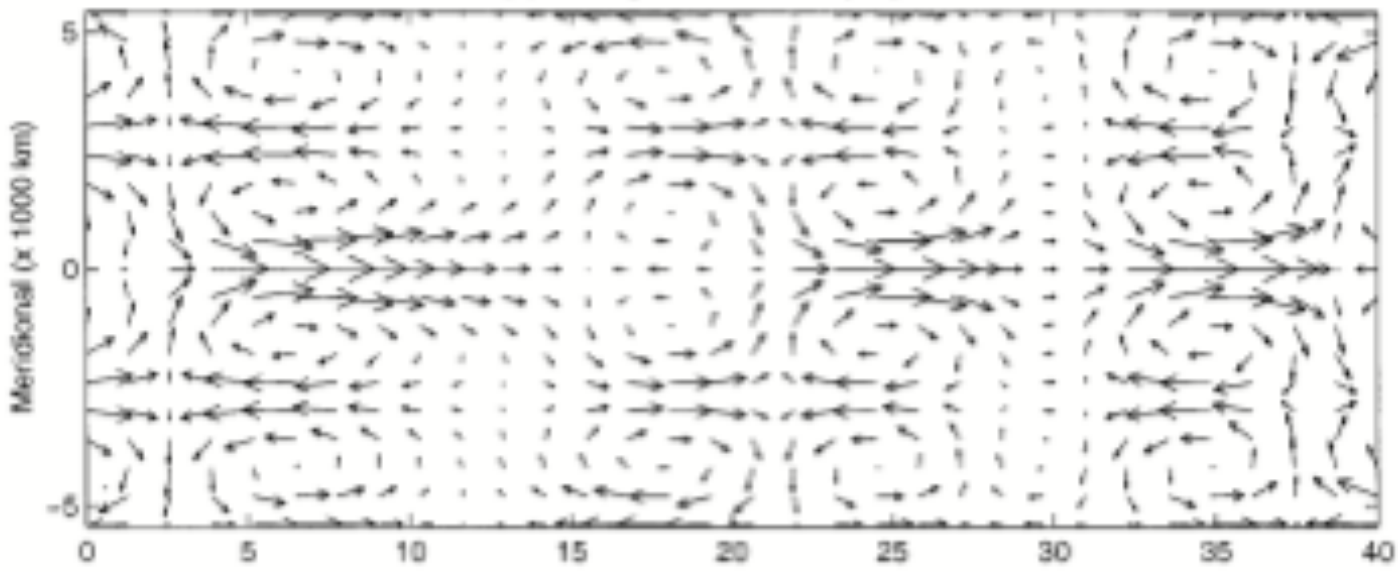

(c) Veiccly at tep of tucposphere

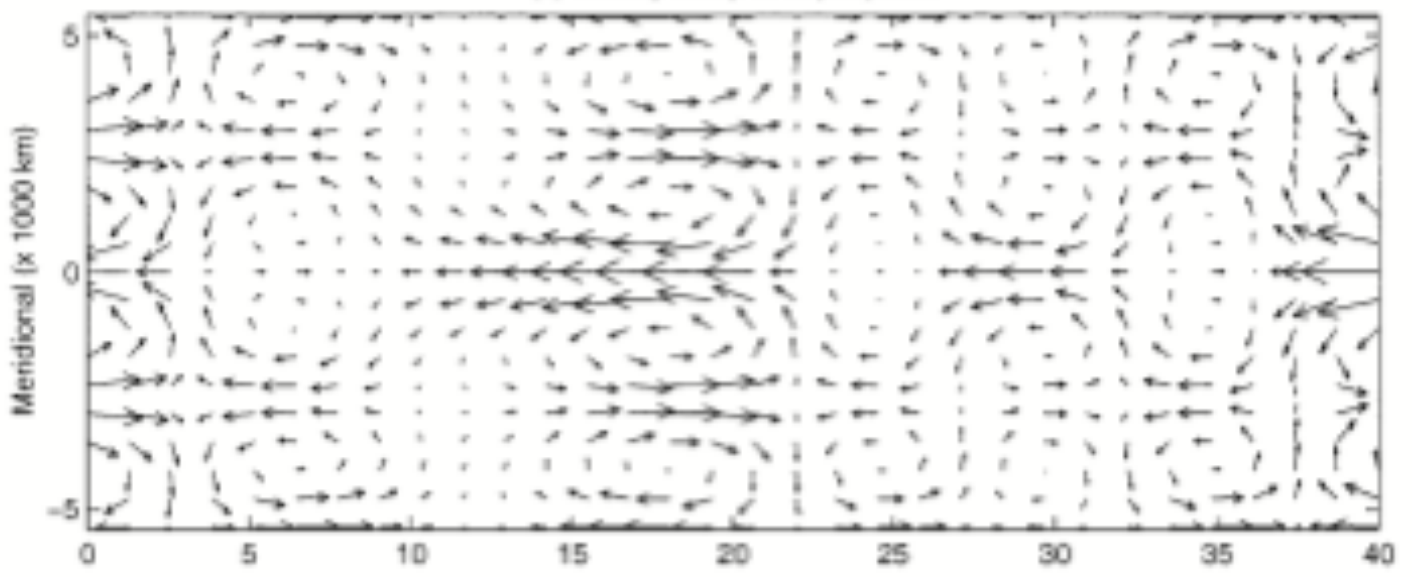

(d) Sposfal Ancileobse, $\mathrm{t}=18$ days

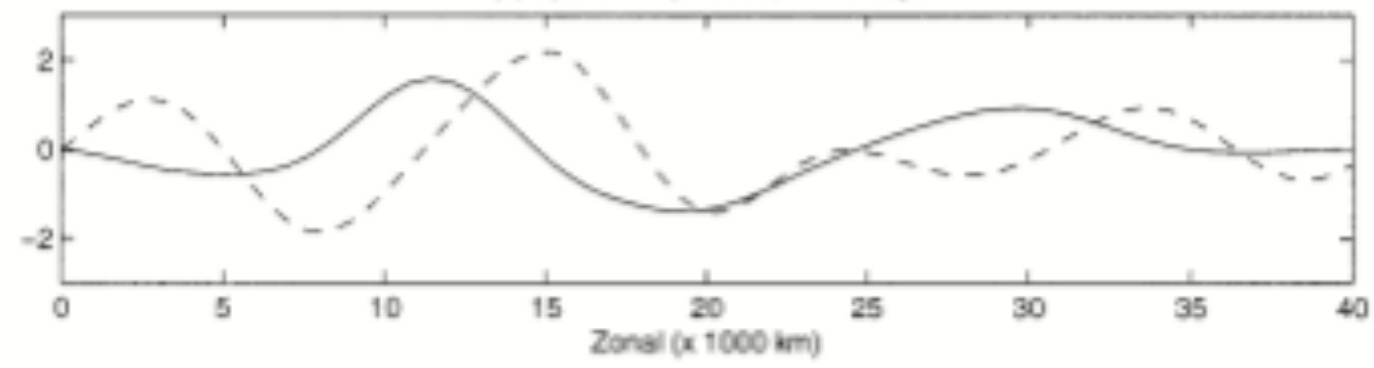

Figure 24. Nonlinear evolution of the barotropic and baroclinic Rossby wave amplitudes with zero baroclinic wave energy initially. $\bar{A}(0)=5, \bar{B}=0, m=1$. Zonal distribution of $A^{\prime}$ (dashed) and $B^{\prime}$ at (a) $t=0$ and (d) $t=18$ days. Total velocity arrows at (b) bottom and (d) top of the troposphere. Adapted from [23]. 


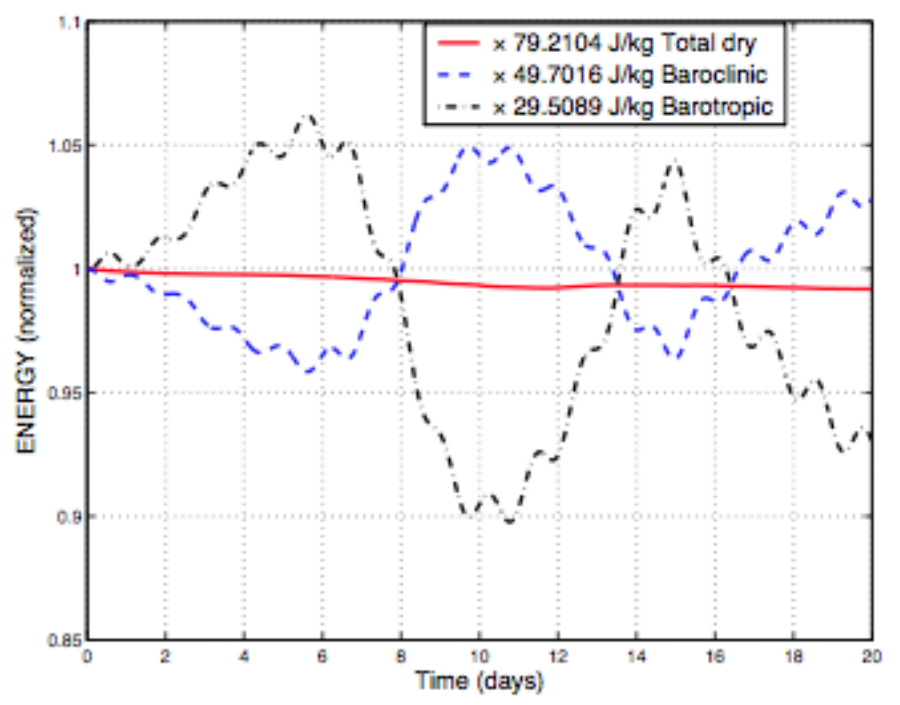

\section{Baroclinic}
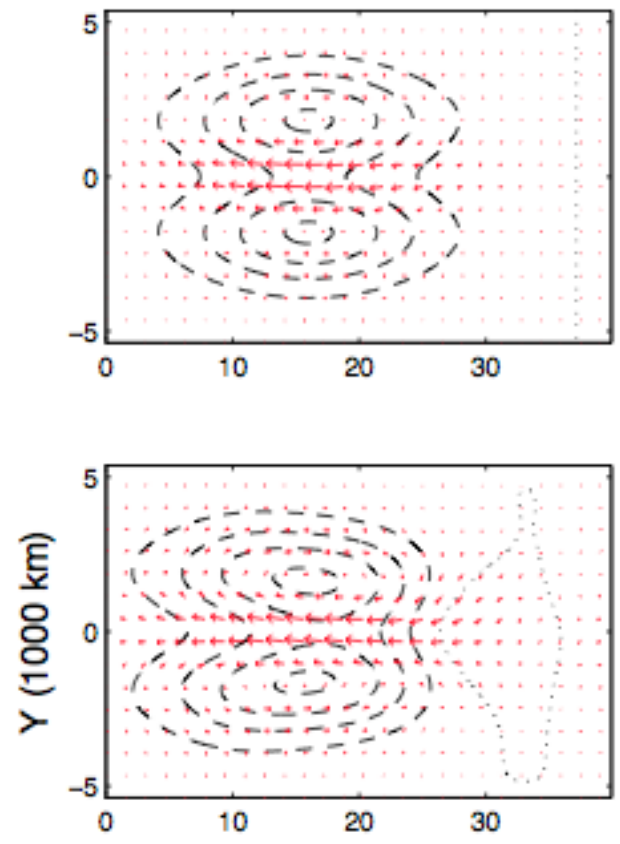

Barotropic
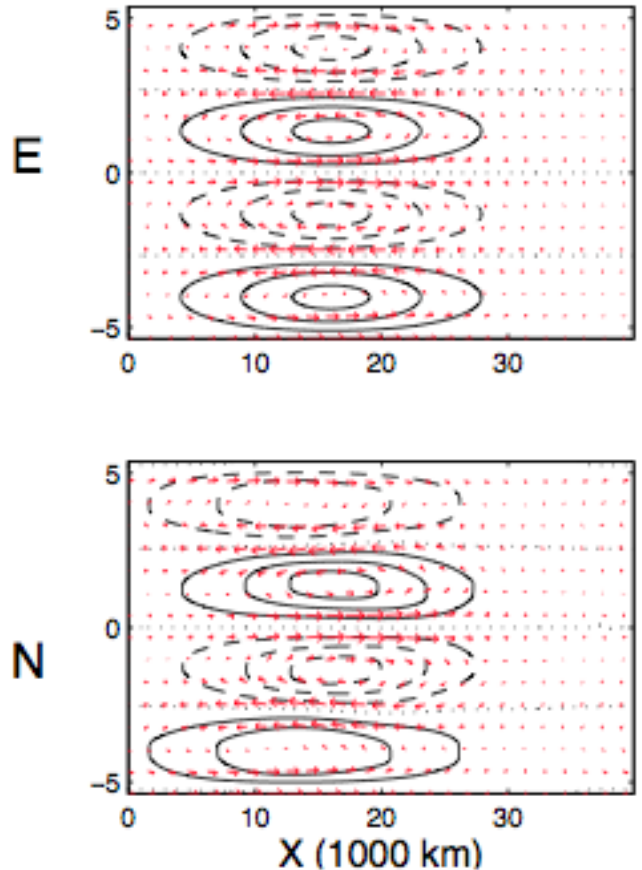

Figure 25. Nonlinear evolution of the solitary-wave solution in the barotropicbaroclinic system (3.3). $m=1, c_{s}=23 \mathrm{~m} \mathrm{~s}^{-1}$.. Zonal distribution of $A^{\prime}$ (dashed) and $B^{\prime}$ at (a) $t=0$ and (d) $t=18$ days. Total velocity arrows at (b) bottom and (d) top of the troposphere. $\bar{A}=\bar{B}=5 \mathrm{~m} \mathrm{~s}^{-1}$. Time series of barotropic, baroclinic, and total energy (top). Structure of the baroclinic (left) and barotropic (right) solution for the solitary wave solution of (6.4) (middle) and numerical solution of (3.3) with same initial data (bottom). Contour of potential temperature and vorticity are shown for the baroclinic and barotropic parts, respectively. Adapted from [43]. 
In this case, (3.3) reduces to the forced vorticity-stream function equation

$$
\frac{\partial \Delta \bar{\psi}}{\partial t}+J(\bar{\psi}, \bar{\Delta} \psi)+\bar{\psi}_{x}=\frac{1}{2} k_{0} \alpha_{0}^{2} y e^{-y^{2}} \sin \left(2 k_{0}\left(x-c_{0} t\right)\right) \equiv F(x, y, t) .
$$

In [112], (6.5) is first solved numerical using the Arakawa Jacobian [113]; a finite differences scheme that conserves both energy and enstrophy. The computational domain is a periodic channel of 40,000 km length and 10,000 km width, centered at the equator. The system is integrated for 100 days. After a small transient period of 5 days, the solution enters a statistical steady state where the barotropic response balances the forcing. A few snapshots of the solution are shown in Figure 26, for the parameter values $\alpha_{0}=10 \mathrm{~m} \mathrm{~s}^{-1}, k_{0}=1$, and $c_{0}=5 \mathrm{~m} \mathrm{~s}^{-1}$.

As we can see from Figure 26, although the forcing is trapped in the vicinity of the equator, the barotropic response is global and reaches the lateral boundaries of the domain. Careful investigation, using spectral analysis, revealed that the solution consists of a phase-locked barotropic wave that moves eastward with the forcing and is equally trapped in the vicinity of the equator and a certain number of barotropic Rossby wave packets whose structure expands laterally to the whole planet.

Inspired by this finding, Ferguson et al. [112], proposed the following ansatz for an analytic solution to the forced barotropic vorticity equation (6.5).

$$
\bar{\psi}=\bar{\psi}_{0}+\sum_{j=1}^{\infty} \bar{\psi}_{j}
$$

where $\bar{\psi}_{0}$ represents the phase-locked wave and $\bar{\psi}_{j}=\sin (y l) \cos (k x-\omega t)$ are Rossby wave packets with a zonal wavenumber $k=2 k_{0}$ and meridional wavenumber $j=1,2, \cdots$. Plugging this ansatz in (6.5) yields [112]

$$
\begin{aligned}
& \left(\frac{\partial \Delta \bar{\psi}_{0}}{\partial t}+\frac{\partial \bar{\psi}_{0}}{\partial x}-F(x, y, t)\right)+\left(J\left(\bar{\psi}_{0}, \Delta \bar{\psi}_{0}\right)+\sum_{i \neq j} J\left(\bar{\psi}_{i}, \Delta \bar{\psi}_{j}\right)\right)=\sum_{j=1}^{\infty}\left(\frac{\partial \Delta \bar{\psi}_{j}}{\partial t}+J\left(\bar{\psi}_{j}, \Delta \bar{\psi}_{j}\right)+\frac{\partial \bar{\psi}_{j}}{\partial x}\right) \\
& \text { Using the fact that } J\left(\bar{\psi}_{j}, \Delta \bar{\psi}_{j}\right)=0 \text { and assuming that } \frac{\partial \Delta \bar{\psi}_{0}}{\partial t}+\frac{\partial \bar{\psi}_{0}}{\partial x}-F(x, y, t)=0 \\
& {[112] \text {, we find }} \\
& \qquad \bar{\psi}_{0}(x, y, t)=[A \cos (k x-\omega t)+B \sin (k x-\omega t)] g(y)
\end{aligned}
$$

where

$$
g(y)=(\sqrt{\pi} / 8)\left[(1-\operatorname{erf}(y+\lambda / 2)) e^{\lambda y}-(1-\operatorname{erf}(-y+\lambda / 2)) e^{-\lambda y}\right] .
$$

The sequence of Rossby wave packets that contribute effectively to the overall solution satisfy $J\left(\bar{\psi}_{0}, \Delta \bar{\psi}_{0}\right)+\sum_{J=1}^{\infty} J\left(\bar{\psi}_{j}, \Delta \bar{\psi}_{j}\right)=0$. It is found in [112] that the numerical solution is formed by the Rossby waves associated with the meridional modes $g(y)$ that carry the energy of the forcing. The mathematical justification for the latter behavior is still an open question though this ansatz is robust to changes in the parameter values $[112]$. 

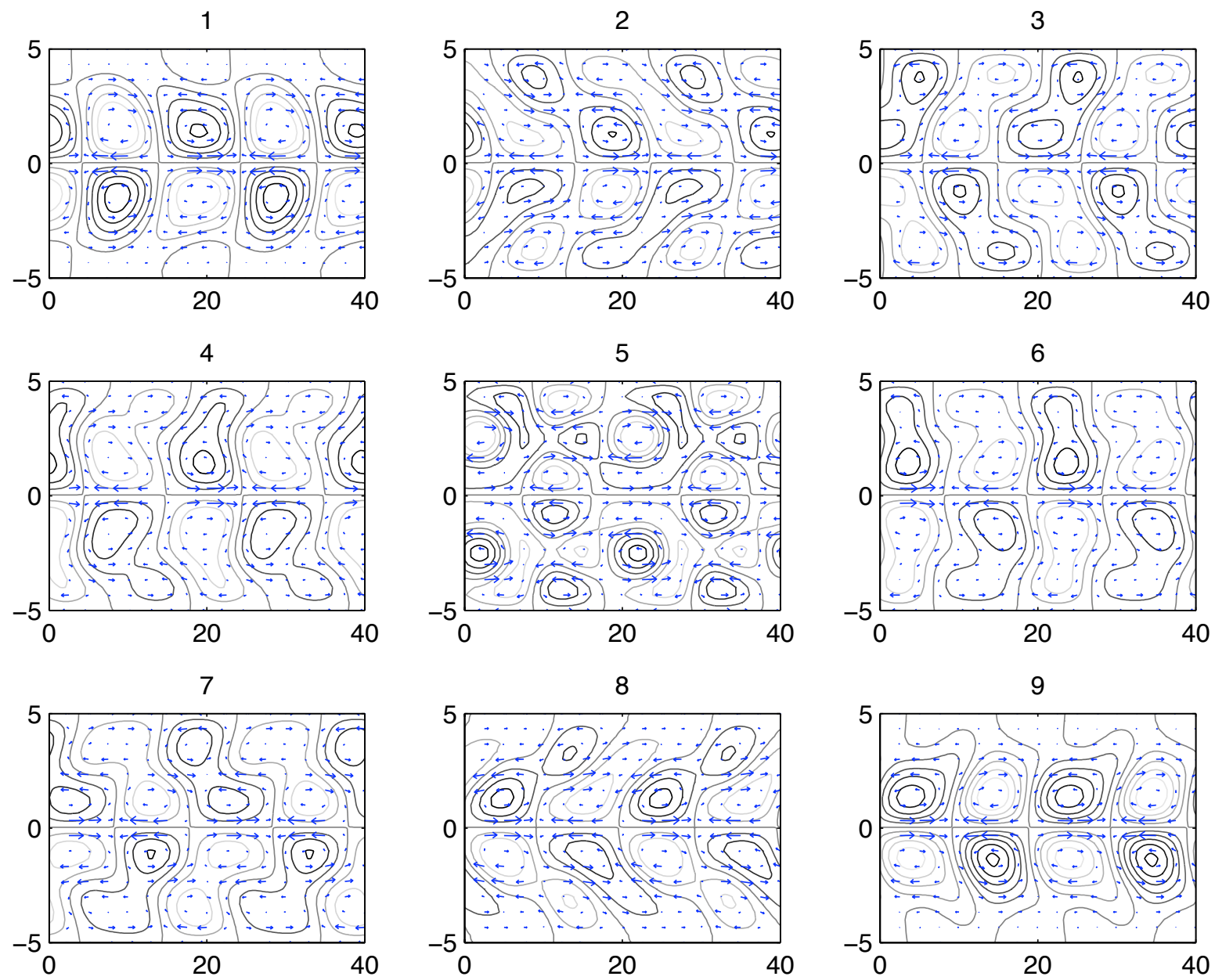

Figure 26. Contours of the stream-function and velocity arrows for the forced barotropic equations (6.5) with $\alpha_{0}=10 \mathrm{~m} / \mathrm{s}, k_{0}=1$, and $c_{0}=5 \mathrm{~m} / \mathrm{s}$. The snapshots are taken successively between times 42 days and 56 days at a roughly 2 days interval. Adapted from [112].

\subsection{Resonant interactions of equatorial waves and the diurnal cycle}

Because of its importance for tropical-extratropical interactions, the excitation of planetary-barotropic Rossby waves by equatorially trapped waves is the subject of many research papers. An interesting way of looking at the problem is by considering resonant triad interactions involving barotropic Rossby waves and equatorially trapped waves. See for example the work of Reznik and Zeitlin [114, 115], Raupp and Silva Dias [116, 117], and Raupp, Tabak, and Milewski [118]. Here we discuss in detail the results of Raupp and Silva Dias in [117] where they considered the case when the resonant triads interact with the diurnal cycle of convection.

Seasonal and diurnal variations of solar radiation are the two major external factors that affect the variability of Earth's weather and climate. The tropical atmosphere responds directly, though in a non trivial fashion, to these periodic forcings by regular 
oscillations in the convective activity and precipitation occurring on the same time scales as the forcing and indirectly by a myriad of chaotic disturbances occurring at various time scales.

As a direct effect of the seasonal cycle, the ITCZ experiences North-South migrations twice a year across the equator while the daily solar variations induce a diurnal cycle in precipitation which typically peaks in the morning over the ocean and in the late afternoon over land. GCMs often do not capture very well both the migration of the ITCZ and the diurnal cycle of convection. The chaotic response of the tropical atmosphere to the seasonal and diurnal cycles of solar radiation are due to the internal modes of variability of the atmosphere and to the way the periodic forcing interacts with these modes, which comprise monsoons and the wide spectrum of convectively coupled waves, including the MJO, that are documented in the previous sections.

The diurnal cycle of precipitation in the multicloud model over both land and ocean is studied by Frenkel et al. [119, 120]. Both the land and the ocean regimes of the diurnal cycle are correctly predicted by the multicloud model $[119,120]$. The interactions of the periodic solar forcing and the internal modes of atmospheric variability is very complex and remains one of the most challenging problems in atmospheric science. The possible interactions between the diurnal cycle and the equatorial waves was the focus of the paper by Raupp and Silva Dias [117], who "aim to present a theory explaining how the diurnal cycle of tropical convection can give rise to low-frequency (intraseasonal) oscillations in the atmosphere". They considered the effect of diurnal cycle forcing on resonant triads involving a barotropic Rossby wave and two equatorially-trapped baroclinic waves and the induced energy exchange between the tropics and the extratropics through this mechanism.

To ease the transition to the original paper for the interested reader, we keep here the exact same notion as in [117] at the risk of having inconsistencies with the rest of this paper. Starting from the equatorial beta-plane primitive equations, with a diabatic forcing in pressure coordinates,

$$
\begin{aligned}
& \frac{\partial u}{\partial t}+\epsilon\left(u \frac{\partial u}{\partial x}+v \frac{\partial u}{\partial y}+F \omega \frac{\partial u}{\partial p}\right)-y v+\frac{\partial \phi}{\partial x}=0 \\
& \frac{\partial v}{\partial t}+\epsilon\left(u \frac{\partial v}{\partial x}+v \frac{\partial v}{\partial y}+F \omega \frac{\partial v}{\partial p}\right)+y u+\frac{\partial \phi}{\partial y}=0 \\
& \frac{\partial u}{\partial x}+\frac{\partial v}{\partial y}+F \frac{\partial \omega}{\partial p}=0 \\
& \frac{\partial}{\partial t} \frac{\partial \phi}{\partial p}+\epsilon\left(u \frac{\partial}{\partial x} \frac{\partial \phi}{\partial p}+v \frac{\partial}{\partial y} \frac{\partial \phi}{\partial p}+F \omega \frac{\partial^{2} \phi}{\partial p^{2}}+\frac{F \omega}{p}(1-\kappa) \frac{\partial \phi}{\partial p}\right)+F \tilde{\bar{\sigma}} \omega=-F \frac{\kappa}{p} \bar{\gamma} Q
\end{aligned}
$$

where $u, v, \omega$ are the zonal, meridional, and pressure-vertical velocity $(\omega=D p / D t)$, $\phi$ is the geopotential and $Q$ is the diabatic (convective) heating. Here $\epsilon$ is the equatorial Rossby number which is considered to be a small parameter and is used for asymptotic expansion. The remaining quantities are constants whose values are specified in the original article. Raupp and Silva Dias assumed periodic boundary conditions in $x$, bounded solutions in $y$, and rigid-lid in the vertical direction. By design, their 
asymptotic set up and time scales mimic the one from [34] discussed earlier in (3.12), (3.13) of the nonlinear Kelvin wave interacting with topography.

Assuming a multi-time scale asymptotic expansion in $\epsilon, \xi=\xi^{0}(x, y, p, t, \epsilon t)+$ $\epsilon \xi^{(1)}(x, y, p, t, \epsilon t)+O\left(\epsilon^{2}\right)$ for the solution $\xi=(u, v, \omega, \phi)$ of $(6.7)$, they arrived at the linear system

$$
\mathcal{J} \xi^{(1)}=N^{(0)}+S
$$

for the first-order perturbation, when grouping terms of the same order in $\epsilon$. Here $\mathcal{J}$ is the linear PDE operator corresponding to the left hand-side of $(6.7), N^{(0)}$ is a vector containing the nonlinear contributions of the leading-order terms $\xi^{(0)}$ and $S$ contains the diabatic forcing, which takes the form

$$
Q(x, y, p, t)=\epsilon Q_{0} H(x, y) q(p) f(t)
$$

where $H(x, y)$ is a simple Gaussian function centered at a specific location mimicking the Amazon forest, $q(p)=\sin \left[\pi\left(p-p_{T}\right) /\left(p-p_{0}\right)\right]$, where $p_{T}$ and $p_{0}$ are respectively the pressure at top and bottom of the troposphere. We note that $q(p)$ has the profile of deep convection that projects solely on the first baroclinic mode. Stratiform and congestus heating are not included and $f(t)$ is a one-day periodic function of time which takes the form of a half sine during one half of the day and zero during the other half. It is expanded in Fourier cosine series,

$$
f(t)=\sum_{j=0}^{\infty} \frac{2}{\pi\left(1-4 j^{2}\right)} \cos \left(\frac{2 \pi j t}{\Delta}\right),
$$

where $\Delta$ is the duration of a day in non-dimensional units [117].

The leading-order solution $\xi^{(0)}$ is expanded in terms of the normal modes of the linear operator $\mathcal{J}$ which are precisely, the barotropic Rossby waves and the whole spectrum of equatorially trapped-baroclinic waves introduced in Section 3:

$$
\xi^{(0)}(x, y, p, t, \tau)=\sum_{a} A_{a}(\tau) \xi_{a}(y) e^{i k_{a} x+i \bar{\omega}_{a} t} G_{a}(p)+c . c .,
$$

where $A_{a}(\tau)$ is the slowly variant amplitude ( $\tau=\epsilon t$ is the slow time) and $\xi_{a}$ is the meridional structure vector-function with $a=(m, k, n, r)$ is an index vector referring to a particular mode with a vertical structure mode $m$, a zonal wavenumber $k$, a meridional index $n$, and the type of wave $r$. Here $m=0$ corresponds to a barotropic mode and $m=1$ represents the first baroclinic mode and $r$ takes the "values" $K, M, R, G$ for Kelvin, mixed-Rossby gravity, Rossby, and inertio-gravity waves, respectively. When the linear equations (6.8) are projected (in the sense of the $L^{2}$ inner product) onto each one of these normal modes, resonant triads emerge, due to the non-linear term $N^{(0)}$. They involve three normal modes $a, b, c$ such that $\bar{\omega}_{a}=\bar{\omega}_{b}+\bar{\omega}_{c}, k_{a}=k_{b}+k_{c}$, and $n_{a}+n_{b}+n_{c}$ is odd, so that the associated $L_{2}$ projection is non trivial. This yields the 
resonant triad ODEs

$$
\begin{aligned}
& c_{a}^{2} \frac{d A_{a}}{d \tau}=A_{b} A_{c} \eta_{a}^{b c}+q_{a} g\left(\bar{\omega}_{a} \pm \frac{2 \pi j_{1}}{\Delta}\right), \\
& c_{b}^{2} \frac{d A_{b}}{d \tau}=A_{a} A_{c}^{*} \eta_{b}^{a c}+q_{b} g\left(\bar{\omega}_{a} \pm \frac{2 \pi j_{2}}{\Delta}\right), \\
& c_{c}^{2} \frac{d A_{c}}{d \tau}=A_{a} A_{b}^{*} \eta_{c}^{a b}+q_{c} g\left(\bar{\omega}_{a} \pm \frac{2 \pi j_{3}}{\Delta}\right) .
\end{aligned}
$$

Here $\eta_{a}^{b c}$ are the non-linear coupling coefficients, $q_{a}$ is the projection of the forcing onto mode $a$ and $j_{1}, j_{2}, j_{3}$ are three arbitrary harmonics of the forcing and the function $g$ satisfies $g(0)=1$ and zero elsewhere.

Given the special form of the imposed heat source, we are concerned here with resonant triads involving two first baroclinic waves and a barotropic Rossby wave. One of the baroclinic waves is assumed to nearly resonate with one harmonic of the diurnal forcing, that is $\bar{\omega}_{a}+2 \pi j / \Delta=O(\epsilon)$ [117]. Raupp and Silva Dias [117] considered the following two main cases: 1$)$ the background forcing, $j=0$-harmonic, resonating with a first baroclinic zonally symmetric geostrophic flow $(k=0)$ and interacting with a barotropic Rossby wave and a mixed Rossby-gravity wave and 2) the first harmonic, $j=1$, (which has a period of one day) forcing a westward inertio-gravity wave and interacting with another inertio-gravity wave and a barotropic Rossby wave. In the first case, the coupling coefficient of the geostrophic mode is zero. This leads to a decoupled equation $c_{c}^{2} d A_{c} / d \tau=q_{c}$, whose solution grows linearly without bounds. The remaining two-by-two system is a harmonic oscillator whose frequency increases linearly with $\tau$ [117]. Thus this asymptotic solution breaks down in the long time. In the second, more interesting case, all three coupling coefficients are non-zero resulting in a complex three-by-three nonlinear system whose solutions achieve non-linear saturation in finite time. It results in interesting long-time oscillations reminiscent of the low-frequency (intraseasonal) oscillations of the tropical atmosphere. The numerical solutions obtained by Raupp and Silva Dias [117] are illustrated in Figure 27 for the sake of completeness. It's interesting to note the stronger initial condition leads to faster oscillations that saturate earlier at a slightly lower energy level. The interested reader is referred to the original paper for a more detailed discussion of the solution procedure and of these solutions.

\subsection{Extra-tropical initiation of the $M J O$}

While a lot of progress is made in our understanding of the propagation and maintenance of the MJO and its dynamical features, very little is known about it is initiation. However, a working hypothesis involves two different mechanisms for MJO initiation: one is internal to the tropics while one is external and originates from the extra tropics. The internal mechanisms may include stochastic effects due to synoptic and/or mesoscale convective activity that projects on and feeds energy to the "MJO mode" [121] and self-organization in the wake of an existing MJO event [20, 122]. The observed tropical-midlatitude interactions on the intraseasonal time scales can be traced back 


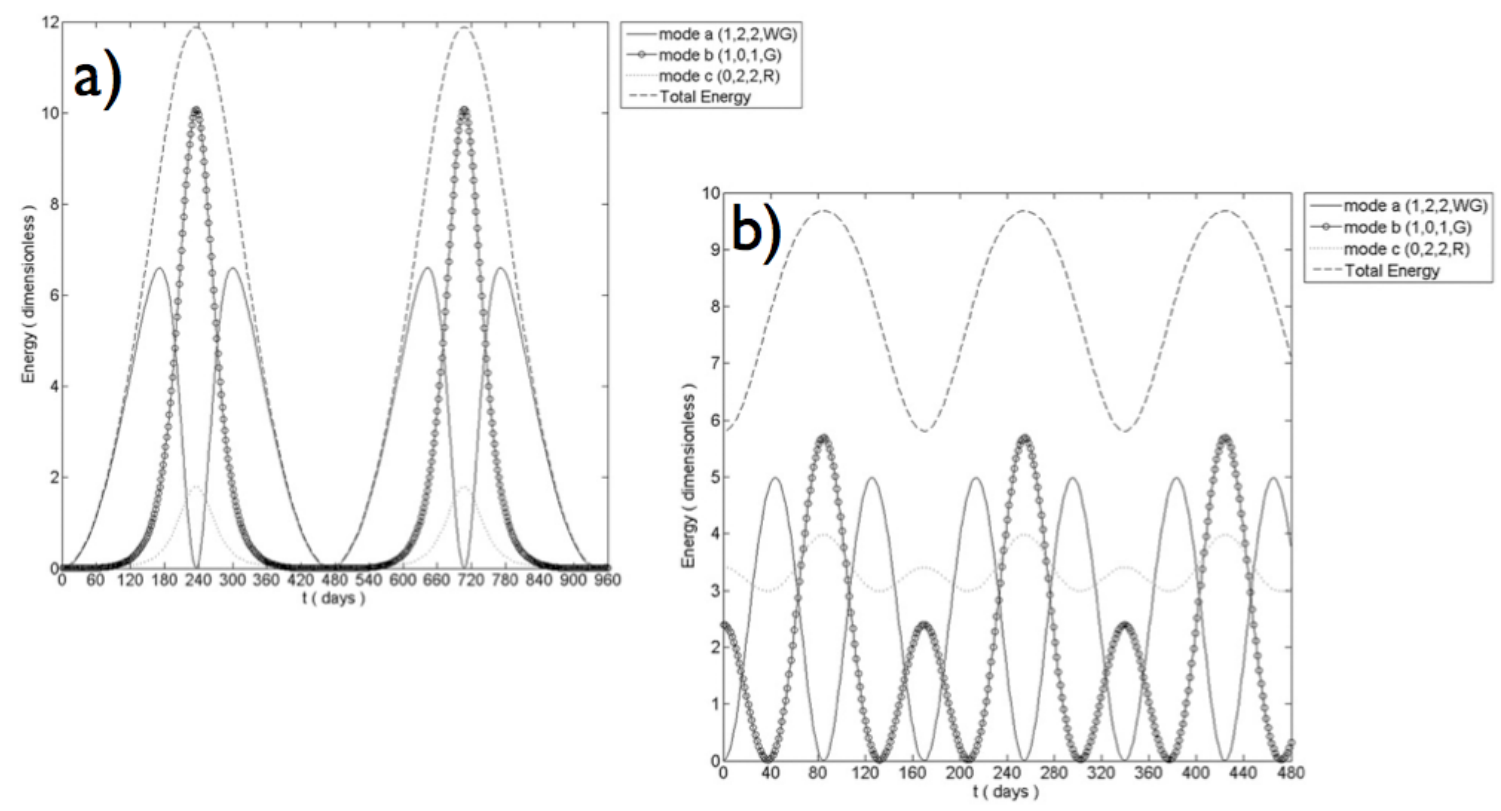

Figure 27. Resonant triads forced by the diurnally varying heat source: Case of two 1st baroclinic gravity waves and a barotropic Rossby wave. (a) $\left|A_{a}(0)\right|=0, \mid A_{b}(0)=$ $0.02|,| A_{c}(0)=0.03 \mid$ and (b) $\left|A_{a}(0)\right|=0,\left|A_{b}(0)=2\right|,\left|A_{c}(0)=0.3\right|$. The forcing resonates with Mode $a$. Adapted from [117].

to the work of Liebmann and Hartmann [123] who showed a clear correlation between fluctuations in $500 \mathrm{mb}$ height in the Northern Hemisphere and outgoing long wave radiation in the tropics thus conjecturing south-east wave propagation. Ray and Zhang [124] demonstrated that an MJO simulated in a channel domain is sensitive to the prescribed winds in the lateral boundary conditions and to the distance of these boundaries from the equator. Lin et al. [125] observed a connection between the North Atlantic Oscillation and the MJO. In this extra-tropical initiation scenario, a possible connection between a planetary scale baroclinic and/or barotropic instability of the basic state is believed to be the main mechanism for the MJO and many other modes of atmospheric variability, including monsoons, storm tracks, etc. [126, 127, 128].

In [126] and [128], a two-layer primitive equation model on the whole sphere is utilized (roughly equivalent to the barotropic-1st baroclinic two mode model discussed in (3.3) above). The model is coupled to a simple convective parameterization (waveCISK in [126] and wave-CISK plus wind induced surface heat exchange-WISHE in [128]) but their results showed that the main-low frequency mode instabilities are insensitive to the convective heating; they are pure dry dynamical-baroclinic-barotropic instabilities.

In [128], for example, Frederiksen's linearized equation for the disturbance potential temperature takes the form

$$
\begin{aligned}
& \frac{\partial \theta}{\partial t}=-J(\bar{\psi}, \theta)-J(\psi, \bar{\theta})-J(\bar{\tau}, \sigma)-J(\tau, \bar{\sigma}) \\
& +\nabla \cdot \bar{\sigma} \nabla \zeta+\nabla \cdot \sigma \nabla \bar{\zeta}-K^{\prime} \nabla^{4} \theta-\bar{s} \nabla^{2} \zeta+C_{0}\left(u_{0} \bar{u}_{0}+\bar{v}_{0} v_{0}\right)\left(q_{s}-\bar{q}\right) .
\end{aligned}
$$


With the convention that the overbar variables denote the basic state values and the "unbarred" the corresponding disturbances, $u_{0}, v_{0}$ are the zonal and meridional velocities in the lower layer, $\psi$ is the vertical mean (barotropic) stream function, $\tau$ is the vertical shear (baroclinic) stream function, $\zeta$ is the lower level velocity potential, and $\sigma$ is the static stability. Capital $J$ denotes the Jacobian determinant and the associated terms represent horizontal advection; also $K^{\prime}$ is a hyperviscosity coefficient, $\bar{s}$ is a wave-CISK parameter and $C_{0}$ is a WISHE parameter which depends on the background lowerlevel zonal wind. They used monthly averaged global fields for January 1979, from the ECMWF analysis data. They considered both the case of a zonally varying and zonally symmetric basic states. The results were found to be qualitatively similar in the two cases. They considered only cases when the moist static stability $\bar{\sigma}_{m}=\bar{\sigma}-\bar{s}>0$ so that wave-CISK is not active. The effect of WISHE is active and the effect of $C_{0} \neq 0$ was mainly to increase the growth rate of the so-called "MJO mode", which appears to be unstable even in the case when the wave-CISK and WISHE parameters are set to zero (dry basic state), and to destabilize a menagerie of large-scale equatorially trapped waves, including, the Kelvin, MRG, inertio-gravity, and Rossby waves. We note however, because their model supports only one cloud type and doesn't take into account the important properties of organized tropical convection, unlike the multicloud model, the vertical structure of these waves is not realistic. The intraseasonal "MJO" mode exhibits a horizontal structure that extends to the whole planet, unlike the MJO in nature which is a tropical signal. The contours of the mean stream function, the upperlevel velocity potential, the vertical shear zonal velocity, and the upper-level divergence are reproduced in Figure 28 for the sake of convenience. We note in particular that the stream function contours display planetary scale vortices that bear some similarities (at least in terms of length scales) to the quadrupole Rossby gyres present in the actual MJO. We note also this mode has an intraseasonal period of roughly 30 days. Thus it wouldn't be surprising if such a mode would play an important role in MJO initiation by resonating (in some fashion) in time and in space with the MJO skeleton or the actual MJO in nature.

\section{Applied math and rigorous PDEs for the Tropics}

\subsection{Precipitation fronts}

As described earlier in section 3.2, precipitation fronts are the large-scale boundaries between moist and dry regions, and a simplified tropical climate model for their behavior has been studied by $[24,26,25,43,55,51,53,54]$. In addition, rigorous theorems on precipitation fronts have been proven by Majda and Souganidis [52].

In [52], Majda and Souganidis prove the existence and uniqueness of precipitation fronts as suitable weak solutions in the zero-relaxation limit $\tau_{c} \rightarrow 0$ of the hyperbolic free boundary value problem. The proof uses energy estimates for first derivatives, which were established in [24] and shown here in (3.23)-(3.24), as well as a new $L^{2}$-contraction 

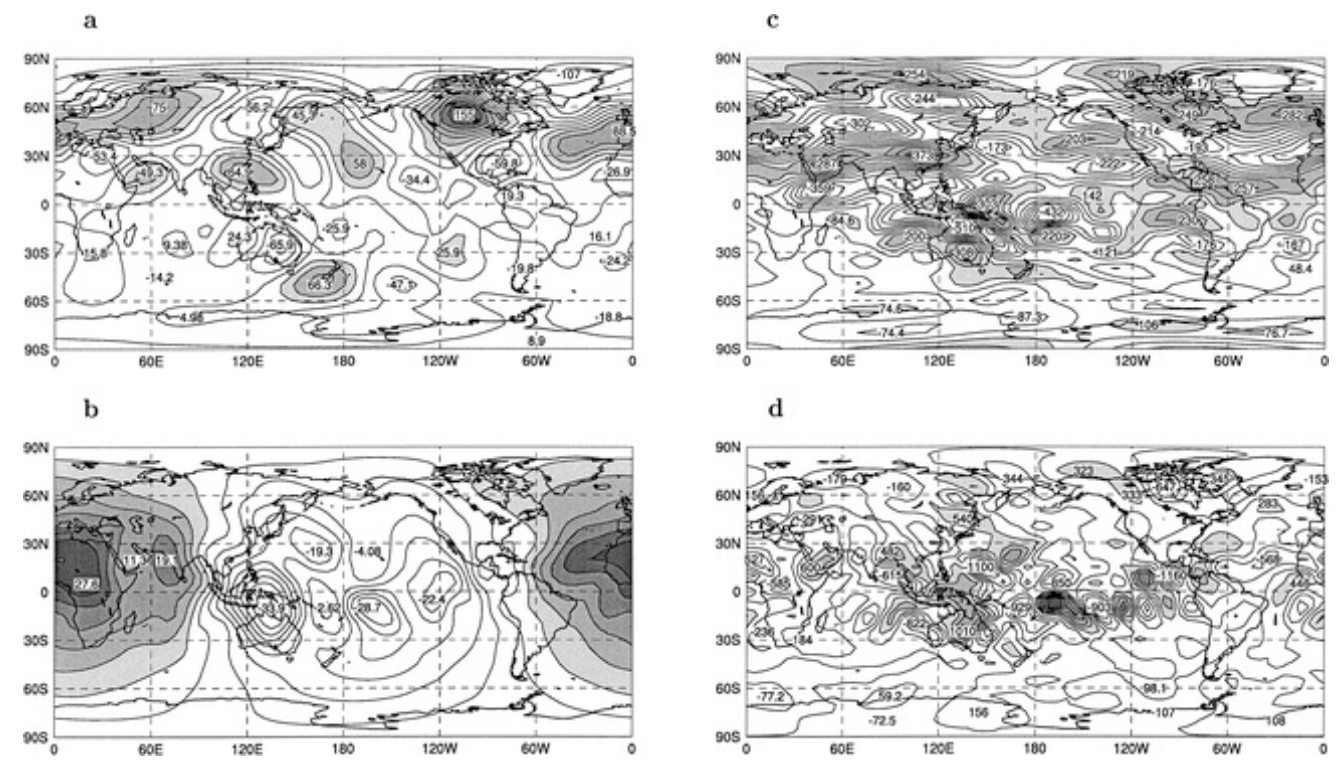

Figure 28. The horizontal structure of the dominant (most unstable) intraseasonal mode in the Frederiksen [128] model. (a) mean stream function, (b) the upperlevel velocity potential, (c) the vertical shear zonal velocity, and (d) the upper-level divergence. Adopted from [128].

property and a new intrinsic characterization of the problem as a variational inequality. The $L^{2}$-contraction property is that

$$
\left\|U^{\epsilon, 1}(\cdot, t)-U^{\epsilon, 2}(\cdot, t)\right\|_{L^{2}} \leq\left\|U^{\epsilon, 1}(\cdot, 0)-U^{\epsilon, 2}(\cdot, 0)\right\|_{L^{2}}
$$

where here $U^{\epsilon, 1}=\left(\mathbf{v}^{\epsilon, 1}, \theta^{\epsilon, 1}, q^{\epsilon, 1}\right)$ and $U^{\epsilon, 2}$ are two solutions, and where this property holds independent of the value of the relaxation timescale $\tau_{c}=\epsilon$ and the $L^{2}$ norm is the moisture weighted energy norm used in (3.22). The variational inequality formally 
states that, for any limit $(\mathbf{v}, \theta, q)$ of $\left(\mathbf{v}^{\epsilon}, \theta^{\epsilon}, q^{\epsilon}\right)$ as $\epsilon \rightarrow 0$,

$$
\begin{aligned}
& \frac{\partial \mathbf{v}}{\partial t}+y \mathbf{v}^{\perp}=\nabla \theta \\
& \frac{\partial}{\partial t}(q+\theta)-(1-\tilde{Q}) \operatorname{div} \mathbf{v}=0 \\
& \frac{\partial q_{e}}{\partial t}+(\tilde{Q}+\alpha) \operatorname{div} \mathbf{v} \leq 0 \\
& q_{e} \leq 0, \\
& \frac{\partial q_{e}}{\partial t}+(\tilde{Q}+\alpha) \operatorname{div} \mathbf{v}=0 \quad \text { a.e. in }\left\{q_{e}<0\right\}
\end{aligned}
$$

where $q_{e}=q-\tilde{q}$ and $\tilde{q}=\hat{q}+\alpha \theta$ is the saturation moisture that was introduced above in (3.2.2). It can then be shown that weak solutions of the variational inequality are unique.

These results were established in [52] in the absence of a barotropic mode. A very interesting problem is to establish existence and uniqueness when the barotropic mode is also included, as written out explicitly in [24].

\subsection{Rigorous multiscale singular limits}

The equatorial shallow water equations (3.11), form a symmetric hyperbolic system, but, because of the large variable-coefficient terms, this system is not covered by the classical Klainerman-Majda theory of singular limits [129, 130]. Nevertheless, several recent results have been established [131, 132, 133].

In $[131,132]$, Dutrifoy and Majda considered the nonlinear shallow water system (3.11) under the assumption that both the Froude number (typical fluid velocity ratio to the gravity wave speed) and the height fluctuations are of order $\epsilon$, in which case (3.11) are rescaled and take the form

$$
\begin{aligned}
& \frac{\partial u}{\partial t}+u \frac{\partial u}{\partial x}+v \frac{\partial u}{\partial y}-\frac{1}{\epsilon}\left(y v+\frac{\partial \theta}{\partial x}\right)=0 \\
& \frac{\partial v}{\partial t}+u \frac{\partial v}{\partial x}+v \frac{\partial v}{\partial y}+\frac{1}{\epsilon}\left(y u-\frac{\partial \theta}{\partial y}\right)=0 \\
& \frac{\partial \theta}{\partial t}+u \frac{\partial \theta}{\partial x}+v \frac{\partial \theta}{\partial y}+\theta\left(\frac{\partial u}{\partial x}+\frac{\partial v}{\partial y}\right)-\frac{1}{\epsilon}\left(\frac{\partial u}{\partial x}+\frac{\partial v}{\partial y}\right)=0
\end{aligned}
$$

By exploiting the special structure hidden in (7.3), these equations can be rewritten as a symmetric hyperbolic system,

$$
\frac{\partial U}{\partial t}+S_{1}(U) \frac{\partial U}{\partial x}+S_{2}(U) \frac{\partial U}{\partial y}+\frac{1}{\epsilon} \mathcal{L} U=0
$$

where $U=((u+\theta) / \sqrt{2},(-u+\theta) / \sqrt{2}, v)$ and $\mathcal{L}$ is a symmetric matrix involving the raising and lowering operators $L_{ \pm}=\partial_{y} \pm y$ from the quantum harmonic oscillator. Since the classical Klainerman-Majda theory of singular limits $[129,130]$ does not directly apply in this case with large variable coefficient terms, Dutrifoy and Majda 
$[131,132]$ introduce a modified Sobolev space $\bar{W}^{s}$, defined as the space of functions $f(x, y) \in L^{2}(\mathbb{T} \times \mathbb{R})$ such that

$$
\sum_{\alpha+\beta+\gamma \leq s}\left\|\partial_{x}^{\alpha} y^{\beta} \partial_{y}^{\gamma} f\right\|_{L^{2}}<\infty
$$

and obtained uniform-in- $\epsilon$ estimates in this modified space.

In [132], Dutrifoy and Majda used fast-wave averaging and showed that zonal jets arise as the singular limit of (7.3). Indeed, one must have

$$
\begin{aligned}
& -y v-\frac{\partial \theta}{\partial x}=0 \\
& y u-\frac{\partial \theta}{\partial y}=0 \\
& \frac{\partial u}{\partial x}+\frac{\partial v}{\partial y}=0
\end{aligned}
$$

if all terms of order $\epsilon^{-1}$ in (7.3) are to vanish. Differentiating the first equation in (7.6) with respect to $y$, the second with respect to $x$ and subtracting gives $-v-y \partial_{y} v-y \partial_{x} u=$ 0 , hence $v=0$ using the third equation. Then the first gives $\partial_{x} \theta=0$, so $\theta$ must be independent of $x$, and so by the third equation $u$ must be independent of $x$ too - i.e., a zonal jet. In [132], the forced version of (7.3) is also considered, and the computation of resonances shows that fast waves may interact with a strong external forcing, introduced to mimic the effects of moisture, to create zonal jets.

In [131], Dutrifoy and Majda consider long-wave-scaled solutions $(u, v, \theta)(\epsilon x, y, t)$. Rescaling (7.3) in the horizontal variable $X=\epsilon x$ gives the long-wave-scaled system,

$$
\begin{aligned}
& \frac{\partial u}{\partial t}+\epsilon u \frac{\partial u}{\partial X}+v \frac{\partial u}{\partial y}-\frac{\partial \theta}{\partial X}-\frac{1}{\epsilon} y v=0 \\
& \frac{\partial v}{\partial t}+\epsilon u \frac{\partial v}{\partial X}+v \frac{\partial v}{\partial y}+\frac{1}{\epsilon}\left(y u-\frac{\partial \theta}{\partial y}\right)=0 \\
& \frac{\partial \theta}{\partial t}+\epsilon u \frac{\partial \theta}{\partial X}+v \frac{\partial \theta}{\partial y}-(1-\epsilon \theta) \frac{\partial u}{\partial X}+\theta \frac{\partial v}{\partial y}-\frac{1}{\epsilon} \frac{\partial v}{\partial y}=0 .
\end{aligned}
$$

Under suitable assumptions on the initial data, solutions to this system converge as $\epsilon \rightarrow 0$ to solutions $(u, \theta)$ of the long-wave equations

$$
\begin{aligned}
& \frac{\partial u}{\partial t}-\frac{\partial \theta}{\partial X}-y v=0 \\
& \frac{\partial \theta}{\partial t}-\frac{\partial u}{\partial X}-\frac{\partial v}{\partial y}=0 \\
& y u-\frac{\partial \theta}{\partial y}=0 .
\end{aligned}
$$

Here, variable $v$ plays the role of a Lagrange multiplier which can be eliminated from the equations using the condition $y u+\partial_{y} \theta=0$, in the same way as the pressure can be eliminated from the incompressible Euler equations using the divergence-free condition. This system is the shallow water version of the fully stratified equatorial long-wave equations shown above in (4.3). See [22] for an elementary discussion with this viewpoint. 
On the other hand, in [133], Dutrifoy, Majda, and Schochet present a simpler proof for balanced initial data by using direct algebraic properties of the fast operator, rather than expanding solutions in a basis of parabolic cylinder functions. This alternative is closer in spirit to the classical theory, uses the weighted spaces in (7.5), and utilizes explicit commutator properties of the raising, lowering, and Hermite operators.

In other related work, both weak and strong convergence results for viscous equatorial shallow-water equations were later obtained by Gallagher and Saint-Raymond [134] by a very different approach following the solution strategy of Leray-Hopf at fixed viscosity and utilizing a detailed study of resonances.

\section{Future perspectives and open problems}

Climate science in the tropics involves a rich variety of physics and math problems. While significant progress has been reviewed here, many long-standing challenges remain in terms of theory, computational modeling, and observations as well. Two of the most challenging aspects are that (i) water vapor and moist convection are strongly coupled with the fluid dynamics and cannot be ignored, and (ii) the range of scales is vast, and different interesting dynamics arise on each set of different scales, in association with a variety of different climate processes.

Coupling with water vapor and moist convection is essential and challenging. For GCMs, this is manifest as the long-standing "cumulus parameterization problem" of representing moist convection as a subgrid-scale process [135]. Some progress on this problem was reviewed above. In particular, the multicloud parameterization of Khouider and Majda reproduces key features of multiscale coherent structures such as CCEWs and the MJO in idealized GCM simulations [49, 136, 50, 95, 20]; a further challenge, which is being pursued by the first two authors, is to use the multicloud parameterization in a full-physics GCM setting. In addition to the multicloud parameterization, superparameterization techniques also reproduce key features of CCEWs and the MJO $[137,19]$, and an open challenge is to design superparameterization algorithms without great computational cost beyond the dry GCM dynamical core [79, 80]. Stochastic multi-cloud models $[96,97,108]$ are an appealing alternative way to achieve this.

For idealized models rather than GCMs, a further challenge with moist convection is to parameterize it in the simplest way possible, while still retaining some of the essential physics. Two such models reviewed above are the models for precipitation fronts and the MJO skeleton. The precipitation fronts model of Frierson, Majda, and Pauluis [24] describes the dynamics of free boundaries between dry and moist regions. The only nonlinearity in the model is a nonlinear switch in the convection parameterization, which is a simplified version of moist convective adjustment present in contemporary GCMs [45, 27]. The MJO skeleton model of Majda and Stechmann [92, 93] predicts the coarsest-scale features of the MJO, in model with a minimal number of parameters and with only a single quadratic nonlinearity. Idealized models such as these offer insight into the complex world of fluid dynamics coupled with moist convection. 
A second challenging aspect for tropical climate science is the vast range of scales involved. As reviewed above, scale-dependent and multiscale models provide a natural framework for understanding the different dynamics and physics on each set of scales. The applications involved the full range of scales from planetary to sub-cloud scales, including the MJO, "self-similarity" in organized tropical convection, squall lines, convective momentum transport, the hurricane embryo, deep and shallow cumulus clouds, and others. Also challenging is the observational analysis of such multiscale systems, and several recent field campaigns have been designed to target this issue [7].

Open problems also remain for rigorous PDEs and applied math, and many of them are suggested by the new PDEs reviewed above. We end with a list of open problems:

(i) The hurricane embryo model of [61] involves a mixed hyperbolic-elliptic system of PDEs. Study the existence and uniqueness of solutions for this system, with active moisture source terms.

(ii) In the tropical-extratropical interactions model of [28], there is degenerate Ekman friction - i.e., the asymptotic model has the property that the dissipation matrix has one eigenvalue which is nearly zero, and hence the dynamics rapidly dissipates flows with pressure at the base of the troposphere and creates barotropic/baroclinic spin up/spin down. While this effect was studied in some situations in [28], much more could be done. What is the role of this degenerate Ekman friction spin-down/spinup in tropical-extratropical interactions? What can be established rigorously for large time behavior?

(iii) Existence and uniqueness of precipitation fronts were established by [52] in the absence of a barotropic mode. An interesting and difficult problem is to establish existence and uniqueness when the barotropic mode is also included, as written out explicitly in [24].

(iv) The rigorous multiscale singular limits established by Dutrifoy and Majda [131, 132] and Dutrifoy, Majda, and Schochet [133] were all for the two-dimensional shallow water case only. Can similar results be established for fully three-dimensional flows? One difficulty is that the nonlinearities in the 3D case can cause a loss of hyperbolicity, which can be seen even in a simple setting with only two vertical baroclinic modes [35] so some vertical viscosity is needed. Can this viscosity be sent to zero in the large scale limit?

(v) In the context of convectively coupled wave-mean flow interaction, it was shown in [75] that amplitude ODEs can capture the behavior of the nonlinear PDEs, including nonlinear oscillations and a Hopf bifurcation. However, a derivation of the amplitude ODEs using systematic asymptotics was not given, due to the complicated form of the nonlinearities in the source terms of the governing equations, including nonlinear switches. Can the amplitude ODEs be derived using systematic asymptotics? This may require a modified version of the governing nonlinear PDEs that does not involve nonlinear switches yet still retains the essential behavior of the multicloud model. 


\section{Acknowledgement}

The research of B.K. is partially supported by a grant from the Natural Sciences and Engineering Research Council of Canada. The research of A. J. M. is partially supported by National Science Foundation grants DMS-0456713 and DMS-1025468 and by the office of Naval Research grants ONR DRI N0014-10-1-0554 and N00014-11-1-0306. The research of S. N. S. is supported by a NSF Mathematical Sciences Postdoctoral Research

Fellowship and a start-up grant from the University of Wisconsin-Madison. The authors would like to thank the associate editor Professor Edriss Titi for his consideration and for inviting them to write this review paper.

\section{References}

[1] A. J. Majda. Real world turbulence and modern applied mathematics. In Mathematics: Frontiers and Perspectives, Amer. Math. Soc. Providence., pages 135-151, 2000.

[2] R. Madden and P. Julian. Detection of a 40-50 day oscillation in the zonal wind in the tropical pacific. J. Atmos. Sci., 28:702-708, 1971.

[3] R. Madden and P. Julian. Description of global-scale circulation cells in the tropics with a 40-50 day period. J. Atmos. Sci., 28:1109-1123, 1972.

[4] C. Zhang. Madden-Julian Oscillation. Reviews of Geophysics, 43:G2003+, June 2005.

[5] W. K. M. Lau and D. E. Waliser. Intraseasonal Variability in the Atmosphere-Ocean Climate System. Springer-Verlag, 2011.

[6] J-L Lin, G. N. Kiladis, B. E. Mapes, K. M. Weickmann, K. R. Sperber, W. Lin, M. C. Wheeler, S. D. Schubert, A. Del Genio, L. J. Donner, S. Emori, J F Gueremy, F. Hourdin, P. J. Rasch, E. Roeckner, and J. F. Scinocca. Tropical intraseasonal variability in 14 IPCC AR4 climate models. Part I: Convective signals. Journal of Climate, 19(12):2665-2690, 2006.

[7] M.W. Moncrieff, M. Shapiro, J Slingo, and F. Molteni. Collaborative research at the intersection of weather and climate. WMO Bulletin, 56:204-211, 2007.

[8] O. Pauluis, A. Czaja, and R. Korty. The global atmospheric circulation on moist isentropes. Science, 321(5892):1075-1078, 2008.

[9] C. R. Mechoso, A. W. Robertson, N. Barth, M. K. Davey, P. Delecluse, P. R. Gent, S. Ineson, B. Kirtman, M. Latif, H. L. Treut, et al. The seasonal cycle over the tropical Pacific in coupled ocean-atmosphere general circulation models. Mon. Wea. Rev., 123(9):2825-2838, 1995.

[10] J.-L. Lin. The double-ITCZ problem in IPCC AR4 coupled GCMs: Ocean-atmosphere feedback analysis. J. Climate, 20(18):4497-4525, 2007.

[11] C. Wang. Atmospheric circulation cells associated with the El Niño-Southern Oscillation. Journal of Climate, 15(4):399-419, 2002.

[12] B. Stevens. Atmospheric moist convection. Annu. Rev. Earth Planet. Sci., 33(1):605-643, 2005.

[13] J. M. Slingo, K. R. Sperber, J. S. Boyle, J.-P. Ceron, M. Dix, B. Dugas, W. Ebisuzaki, J. Fyfe, D. Gregory, J.-F. Gueremy, J. Hack, A. Harzallah, P. Inness, A. Kitoh, W. K.-M. Lau, B. McAvaney, R. Madden, A. Matthews, T. N. Palmer, C.-K. Parkas, D. Randall, and N. Renno. Intraseasonal oscillations in 15 atmospheric general circulation models: results from an AMIP diagnostic subproject. Climate Dynamics, 12:325-357, April 1996.

[14] M. W. Moncrieff and E. Klinker. Organized convective systems in the tropical western Pacific as a process in general circulation models: A TOGA COARE case-study. Quarterly Journal of the Royal Meteorological Society, 123:805-827, April 1997.

[15] D. Kim, K. Sperber, W. Stern, D. Waliser, I.-S. Kang, E. Maloney, W. Wang, K. Weickmann, J. Benedict, M. Khairoutdinov, et al. Application of MJO simulation diagnostics to climate models. J. Climate, 22(23):6413-6436, 2009. 
[16] W. W. Grabowski and P. K. Smolarkiewicz. CRCP: a Cloud Resolving Convection Parameterization for modeling the tropical convecting atmosphere. Physica D: Nonlinear Phenomena, 133:171-178, 1999.

[17] W. W. Grabowski. Coupling cloud processes with the large-scale dynamics using the cloudresolving convection parameterization (CRCP). J. Atmos. Sci., 58:978-997, May 2001.

[18] D. Randall, M. Khairoutdinov, A. Arakawa, and W. Grabowski. Breaking the cloud parameterization deadlock. Bull. Amer. Meteor. Soc., 84:1547-1564, November 2003.

[19] J.J. Benedict and D.A. Randall. Structure of the Madden-Julian Oscillation in the Superparameterized CAM. J. Atmos. Sci., 66(11):3277-3296, 2009.

[20] B. Khouider, A. St-Cyr, A. J. Majda, and J. Tribbia. The MJO and convectively coupled waves in a coarse-resolution GCM with a simple multicloud parameterization. J. Atmos. Sci., 68(2):240-264, 2011.

[21] G. N. Kiladis, M. C. Wheeler, P. T. Haertel, K. H. Straub, and P. E. Roundy. Convectively coupled equatorial waves. Rev. Geophys., 47:RG2003, doi:10.1029/2008RG000266, 2009.

[22] A. J. Majda. Introduction to PDEs and Waves for the Atmosphere and Ocean, volume 9 of Courant Lecture Notes in Mathematics. American Mathematical Society, Providence, 2003.

[23] A. Majda and J. Biello. The nonlinear interaction of barotropic and equatorial baroclinic Rossby waves. J. Atmos. Sci., 60:1809-1821, 2003.

[24] D. Frierson, A. Majda, and O. Pauluis. Dynamics of precipitation fronts in the tropical atmosphere. Comm. Math. Sciences, 2:591-626, 2004.

[25] B. Khouider and A. J. Majda. A non-oscillatory well balanced scheme for an idealized tropical climate model. Part I: Algorithm and validation. Theor. Comp. Fluid Dyn., 19:331-354, 2005.

[26] S. N. Stechmann and A. J. Majda. The structure of precipitation fronts for finite relaxation time. Theor. Comp. Fluid Dyn., 20:377-404, 2006.

[27] J. D. Neelin and N. Zeng. A quasi-equilibrium tropical circulation model: Formulation. J. Atmos. Sci., 57:1741-1766, 2000.

[28] J. Biello and A. Majda. Boundary layer dissipation and the nonlinear interaction of equatorial baroclinic barotropic rossby waves. Geophysical and Astrophysical Fluid Dynamics, 98:85-127, 2004.

[29] J. Biello and A. Majda. The effect of meridional and vertical shear on the interaction of equatorial baroclinic and barotropic rossby waves. STUDIES IN APPLIED MATHEMATICS, 112:341390, 2004.

[30] A. E. Gill. Atmosphere-Ocean Dynamics. Academic, San Diego, Calif., 1982.

[31] J.h Pedlosky. Geophysical Fluid Dynamics. Springer, 1987.

[32] J. Holton. An introduction to dynamic meterology. Elsevier Academic Press, 2004.

[33] T. Matsuno. Quasi-geostrophic motions in the equatorial area. J. Met. Jap., 44:25-41, 1966.

[34] A. J. Majda, R. Rosales, E. G. Tabak, and C. V. Turner. Interaction of large-scale equatorial waves and dispersion of Kelvin waves through topographic resonances. J. Atmos. Sci., 56:No. 24, 1999.

[35] S. N. Stechmann, A. J. Majda, and B. Khouider. Nonlinear dynamics of hydrostatic internal gravity waves. Theor. Comp. Fluid Dyn., 22:407-432, 2008.

[36] Y. Han and B. Khouider. Convectively coupled waves in a sheared environment. J. Atmos. Sci., 67:2913-2942, September 2010.

[37] G. Strang. On the construction and comparison of difference schemes. SIAM J. Num. Anal., 5:506-517, 1998.

[38] H. Nessyahu and E. Tadmor. Non-oscillatory central differencing for hyperbolic conservation laws. J. Comput. Phys., 87(2):408-463, 1990.

[39] G. S. Jiang and E. Tadmor. Non-oscillatory central differencing for multidimentional hyperbolic conservation laws. SIAM J. Sci. Comp., 19(6):1892-1917, 1998.

[40] S. N. Stechmann and A. J. Majda. Gravity waves in shear and implications for organized convecion. J. Atmos. Sci., 66:2579-2599, 2009. 
[41] W. W. Grabowski. Toward cloud resolving modeling of large-scale tropical circulations: A simple cloud microphysics parameterization. Journal of the Atmospheric Sciences, 55:3283-3298, 1998.

[42] X. Wu and X. Li. A review of cloud-resolving model studies of convective processes. Advances Atmospheric Sciences, 55(17):2693-2714, 1998.

[43] B. Khouider and A. J. Majda. A non-oscillatory well balanced scheme for an idealized tropical climate model. Part II: Nonlinear coupling and moisture effects. Theor. Comp. Fluid Dyn., 19:355-375, 2005.

[44] B. Khouider and A. J. Majda. Multicloud convective parametrizations with crude vertical structure. Theor. Comp. Fluid Dyn., 20:351?375, 2006.

[45] A. K. Betts and M. J. Miller. A new convective adjustment scheme. Part II: Single column tests using GATE wave, BOMEX, atex and arctic air-mass data sets. Quarterly Journal of the Royal Meteorological Society, 112(473):693-709, 1986.

[46] J. D. Neelin and I. M. Held. Modelling tropical convergence based on the moist static energy budget. Mon. Wea. Rev., 115:3-12, 1987.

[47] Y. N. Takayabu. Large-scale cloud disturbances associated with equatorial waves. Part I: Spectral features of the cloud disturbances. J. Meteor. Soc. Japan, 72:433-448, 1994.

[48] M. Wheeler and G. N. Kiladis. Convectively coupled equatorial waves: Analysis of clouds and temperature in the wavenumber-frequency domain. J. Atmos. Sci., 56(3):374-399, 1999.

[49] B. Khouider and A. J. Majda. A simple multicloud parametrization for convectively coupled tropical waves. Part I: Linear analysis. J. Atmos. Sci., 63:1308-1323, 2006.

[50] B. Khouider and A. J. Majda. Multicloud models for organized tropical convection: Enhanced congestus heating. J. Atmos. Sci., 65:897-914, 2008.

[51] J. Dias and O. Pauluis. Convectively coupled waves propagating along an equatorial itcz. $J$. Atmos. Sci., 66:2237-2255, 2009.

[52] A. J. Majda and P. E. Souganidis. Existence and uniqueness of weak solutions for precipitation fronts: A novel hyperbolic free boundary problem in several space variables. Comm. Pure Appl. Math., 63(10):1351-1361, 2010.

[53] F. Bouchut, J. Lambaerts, G. Lapeyre, and V. Zeitlin. Fronts and nonlinear waves in a simplified shallow-water model of the atmosphere with moisture and convection. Physics of Fluids, 21:116604, 2009.

[54] J. Lambaerts, G. Lapeyre, V. Zeitlin, and F. Bouchut. Simplified two-layer models of precipitating atmosphere and their properties. Physics of Fluids, 23:046603, 2011.

[55] O. Pauluis, D. Frierson, and A. Majda. Precipitation fronts and the reflection and transmission of tropical disturbances. Q. J. R. Meteorol. Soc., 134:913?930, 2008.

[56] J. G. Charney. A note on large-scale motions in the tropics. J. Atmos. Sci., 20:607-608, 1963.

[57] I. M. Held and B. J. Hoskins. Large-scale eddies and the general circulation of the troposphere. Advances in Geophysics, 28:3-31, 1985.

[58] A. H. Sobel, J. Nilsson, and L. M. Polvani. The weak temperature gradient approximation and balanced tropical moisture waves. J. Atmos. Sci., 58(23):3650-3665, 2001.

[59] A. J. Majda and R. Klein. Systematic multiscale models for the Tropics. J. Atmos. Sci., 60:393408, January 2003.

[60] A. J. Majda, M. Mohammadian, and Y. Xing. Vertically sheared horizontal flow with mass sources: a canonical balanced model. Geophys. Astrophys. Fluid Dynam., 102(6):543-591, 2008.

[61] A. J. Majda, Y. Xing, and M. Mohammadian. Moist multi-scale models for the hurricane embryo. J. Fluid Mech., 657:478-501, 2010.

[62] P. F. Embid and A. J. Majda. Low Froude number limiting dynamics for stably stratified flow with small or finite Rossby numbers. Geophys. Astrophys. Fluid Dynam., 87(1-2):1-50, 1998.

[63] A.E. Gill. Some simple solutions for heat-induced tropical circulation. Q. J. Royal Meteor. Soc., 106(449):447-462, 1980. 
[64] A. Majda and J. Biello. A multi-scale model for the intraseasonal oscillation. Proc. Nat. Acad. Sci., 101:4736-4741, 2004.

[65] J. Biello and A. Majda. A multi-scale model for the madden-julian oscillation. J. Atmos. Sci., 62:1694-1721, 2005.

[66] B. Mapes, S. Tulich, J. Lin, and P. Zuidema. The mesoscale convection life cycle: Building block or prototype for large-scale tropical waves? Dynamics of Atmospheres and Oceans, 42(1-4):3 $-29,2006$.

[67] G. I. Barenblatt. Scaling. Cambridge University Press, 2003.

[68] A. J. Majda. New multi-scale models and self-similarity in tropical convection. J. Atmos. Sci., 64:1393-1404, 2007.

[69] A. J. Majda. Multiscale models with moisture and systematic strategies for superparameterization. J. Atmos. Sci., 64:2726-2734, 2007.

[70] R. Klein. Asymptotic analyses for atmospheric flows and the construction of asymptotically adaptive numerical methods. Z. Angew. Math. Mech., 80:765-777, 2000.

[71] R. Klein. Scale-dependent models for atmospheric flows. Annu. Rev. Fluid Mech., 42:249-274, 2010.

[72] J. A. Biello and A. J. Majda. Intraseasonal multi-scale moist dynamics of the tropical troposphere. Commun. Math. Sci., 8:519-540, 2010.

[73] A. J. Majda and S. N. Stechmann. A simple dynamical model with features of convective momentum transport. Journal of the Atmospheric Sciences, 66(2):373-392, 2009.

[74] B. Khouider, Y. Han, A. Majda, and S. Stechmann. Multi-scale waves in an MJO background and CMT feedback. J. Atmos. Sci., 69:915-933, 2012.

[75] S. N. Stechmann, A. J. Majda, and D. Skjorshammer. Convectively coupled wave environment interactions. Theor. Comp. Fluid Dyn., page submitted, 2012.

[76] M. W. Moncrieff. Analytic representation of the large-scale organization of tropical convection. J. Atmos. Sci., 61:1521-1538, July 2004.

[77] J. A. Biello, A. J. Majda, and M. W. Moncrieff. Meridional momentum flux and superrotation in the multi-scale IPESD MJO model. J. Atmos. Sci., 64(5):1636-1651, 2007.

[78] R. Caballero and M. Huber. Spontaneous transition to superrotation in warm climates simulated by CAM3. Geophys. Res. Lett., 37(11):L11701, 2010.

[79] Y. Xing, A. J. Majda, and W. W. Grabowski. New efficient sparse space-time algorithms for superparameterization on mesoscales. Monthly Weather Review, 137:4307-4324, 2009.

[80] A. J. Majda and M. J. Grote. Mathematical test models for superparametrization in anisotropic turbulence. Proc. Natl. Acad. Sci., 106(14):5470-5474, 2009.

[81] M. T. Montgomery, M. E. Nicholls, T. A. Cram, and A. B. Saunders. A vortical hot tower route to tropical cyclogenesis. J. Atmos. Sci., 63(1):355-386, 2006.

[82] R. A. Houze, Jr. Mesoscale convective systems. Rev. Geophys., 42:G4003+, December 2004.

[83] A. J. Majda and Y. Xing. New multi-scale models on mesoscales and squall lines. Commun. Math. Sci., 8:113-144, 2010.

[84] R. Klein and A. Majda. Systematic multiscale models for deep convection on mesoscales. Theor. Comp. Fluid Dyn., 20:525-551, 2006.

[85] D. Ruprecht, R. Klein, and A. J. Majda. Modulation of internal gravity waves in a multiscale model for deep convection on mesoscales. J. Atmos. Sci., 67(8):2504-2519, 2010.

[86] S. N. Stechmann and B. Stevens. Multiscale models for cumulus cloud dynamics. J. Atmos. Sci., 67:3269-3285, 2010.

[87] B. Stevens. Bulk boundary-layer concepts for simplified models of tropical dynamics. Theor. Comp. Fluid Dyn., 20(5):279-304, 2006.

[88] M. L. Waite and B. Khouider. Boundary layer dynamics in a simple model for convectively coupled gravity waves. J. Atmos. Sci., 66:2780-2795, 2009.

[89] O. Pauluis and J. Schumacher. Idealized moist Rayleigh-Bénard convection with piecewise linear equation of state. Commun. Math. Sci, 8:295-319, 2010. 
[90] O. Pauluis and J. Schumacher. Self-aggregation of clouds in conditionally unstable moist convection. Proc. Natl. Acad. Sci. USA, 108:12623-12628, 2011.

[91] A. Z. Owinoh, B. Stevens, and R. Klein. Multiscale asymptotics analysis for the mesoscale dynamics of cloud-topped boundary layers. J. Atmos. Sci., 68:379-402, 2011.

[92] A. J. Majda and S. N. Stechmann. The skeleton of tropical intraseasonal oscillations. Proc. Nat. Acad. Sci., 106(21):8417-8422, 2009.

[93] A. J. Majda and S. N. Stechmann. Nonlinear dynamics and regional variations in the MJO skeleton. J. Atmos. Sci., 68:3053-3071, 2011.

[94] A. J. Majda and S. N. Stechmann. Multiscale theories for the MJO. In W. K. M. Lau and D. E. Waliser, editors, Intraseasonal Variability in the Atmosphere-Ocean Climate System. Springer, Berlin, 2011.

[95] B. Khouider and A. J. Majda. Equatorial convectively coupled waves in a simple multicloud model. J. Atmos. Sci., 65:3376-3397, 2008.

[96] B. Khouider, J. Biello, and A. J. Majda. A stochastic multicloud model for tropical convection. Comm. Math. Sci., 8(1):187-216, 2010.

[97] Y. Frenkel, A. J. Majda, and B. Khouider. Using the stochastic multicloud model to improve tropical convective parameterization: A paradigm example. J. Atmos. Sci., 69:1080-1105, 2012.

[98] A. J. Majda, S. N. Stechmann, and B. Khouider. Madden-julian oscillation analog and intraseasonal variability in a multicloud model above the equator. Proc. Nat. Acad. Sci., 104:9919-9924, 2007.

[99] J.M. Dennis, A. Fournier, W. Spotz, A. St-Cyr, M. Taylor, S. J. Thomas, and H.M. Tufo. High resolution mesh convergence properties and parallel efficiency of a spectral element atmospheric dynamical core. Int. J. High Perform. Comput. Appl., Special Issue on Climate Modeling. Eds. J.B. Drake, P. Jones, and G. Carr., 19:225-245, 2005.

[100] M.A. Taylor, J. Edwards, and A. St-Cyr. Petascale atmospheric models for the community climate system model: New developments and evaluation of scalable dynamical codes. $J$. Phys. Conf. Ser., 125:012023, 2008.

[101] A. Kasahara and K. Puri. Spectral representation of three-dimensional global data by expansion in normal mode functions. Mon. Weather Review, 109:37-51, 1981.

[102] M. A. LeMone. Momentum transport by a line of cumulonimbus. J. Atmos. Sci., 40:1815-1834, 1983.

[103] M. W. Moncrieff. A theory of organized steady convection and its transport properties. Quart. J. Roy. Meteor. Soc., 107:29?50, 1981.

[104] M. W. Moncrieff. Organized convective systems: Archetypical dynamical models, mass and momentum flux theory, and parameterization. Quart. J. Roy. Meteor. Soc., 118:819-850, 1992.

[105] M. A. LeMone and M. W. Moncrieff. Momentum and mass transport by convective bands: Comparisons of highly idealized dynamical models to observations. J. Atmos. Sci., 51:281305, 1994.

[106] W. W. Tung and M. Yanai. Convective momentum transport observed during the TOGA COARE IOP. Part I: General features. J. Atmos. Sci., 59:1857-1871, 2002.

[107] W. W. Tung and M. Yanai. Convective momentum transport observed during the TOGA COARE IOP. Part II: Case studies. J. Atmos. Sci., 59:2535-2549, 2002.

[108] A. J. Majda and S. N. Stechmann. Stochastic models for convective momentum transport. Proceedings of the National Academy of Sciences, 105(46):17614-17619, 2008.

[109] B. Khouider, Y. Han, and J. Biello. Convective momentum transport in a simple multicloud model. J. Atmos. Sci., 69(1):281-302, 2012.

[110] W. C. Chao. On the origin of the tropical intraseasonal oscillation. J. Atmos. Sci., 44:1940-1949, 1987.

[111] R. H. Johnson, T. M. Rickenbach, S. A. Rutledge, P. E. Ciesielski, and W. H. Schubert. Trimodal 
characteristics of tropical convection. Journal of Climate, 12(8):2397-2418, 1999.

[112] J. Ferguson, B. Khouider, and M. Namazi. Two-way interactions between between equatorially trapped waves and the barotropic flow. Chinese Annals of Mathematics. Series B, 30:539-568, 2010.

[113] A. Arakawa. Computational design for lon-term numerical integration of the equations of fluid motion: Two-dimensional incompressible flow, Part I. J. Comput. Phys., 1:119-143, 1966.

[114] G. M. Reznik and V. Zeitlin. Resonant excitation of Rossby waves in the equatorial waveguide and their nonlinear evolution. Phys. Rev. Lett., 96(3):34502, 2006.

[115] G. M. Reznik and V. Zeitlin. Interaction of free Rossby waves with semi-transparent equatorial waveguide. Part 1. Wave triads. Physica D: Nonlinear Phenomena, 226(1):55-79, 2007.

[116] C. F. M. Raupp and P. L. Silva Dias. Dynamics of resonantly interacting equatorial waves. Tellus, 58A:263-279, 2006.

[117] C. F. M. Raupp and P. L. Silva Dias. Resonant wave interactions in the presence of a diurnally varying heat source. J. Atmos. Sci., 66:3165-3183, 2009.

[118] C. F. M. Raupp, E. G. Tabak, and P. Milewski. Resonant wave interactions in the equatorial waveguide. J. Atmos. Sci., 65:263-279, 2008.

[119] Y. Frenkel, B. Khouider, and A. J. Majda. Simple multicloud models for the diurnal cycle of tropical precipitation. Part I: Formulation and the case of the tropical oceans. J. Atmos. Sci., 68:2169-2190, 2011.

[120] Y. Frenkel, B. Khouider, and A. J. Majda. Simple multicloud models for the diurnal cycle of tropical precipitation. Part II: The continental regime. J. Atmos. Sci., 68:2192-2207, 2011.

[121] C. Zhang and J. Ling. Potential vorticity of the Madden-Julian oscillation. J. Atmos. Sci., 69:65-78, 2012.

[122] M. Yanai, B. Chen, and W. Tung. The Madden-Julian oscillation observed during the TOGA COARE IOP: Global view. J. Atmos. Sci., 57:2374-2396, 2000.

[123] B. Liebmann and D. Hartmann. An observational study of tropical-midlatitude interaction on intraseasonal time scales during winter. J. Atmos. Sci., 41:3333-3350, 1984.

[124] P. Ray and C. Zhang. A case study of the mechanics of extratropical influence on the initiation of the madden-julian oscillation. J. Atmos. Sci., 67:515-528, 2010.

[125] H. Lin, G. Brunet, and J. Derome. An observed connection between the north atlantic oscillation and the madden-julian oscillation. J. Climate, 22:364-380, 2009.

[126] J. S. Frederiksen and C. S. Frederiksen. Monsoon disturbances, intraseasonal oscillations, teleconnection patterns, blocking, and storm tracks of the global atmosphere during january 1979: Linear theory. J. Atmos. Sci., 50:1349-1372, 1993.

[127] D. M. Strauss and R. S. Lindzen. Planetary-scale baroclinic instability and the mjo. J. Atmos. Sci., 57:3609-3626, 2000.

[128] J. S. Frederiksen. Genesis of intraseasonal oscillations and equatorial waves. J. Atmos. Sci., 59:2761-2781, 2002.

[129] S. Klainerman and A. Majda. Singular limits of quasilinear hyperbolic systems with large parameters and the incompressible limit of compressible fluids. Comm. Pure Appl. Math., 34(4):481-524, 1981.

[130] S. Klainerman and A. Majda. Compressible and incompressible fluids. Comm. Pure Appl. Math., 35(5):629-651, 1982.

[131] A. Dutrifoy and A. Majda. The dynamics of equatorial long waves: a singular limit with fast variable coefficients. Commun. Math. Sci., 4(2):375-397, 2006.

[132] A. Dutrifoy and A. Majda. Fast wave averaging for the equatorial shallow water equations. Communications in Partial Differential Equations, 32:1617-1642, 2007.

[133] A. Dutrifoy, A. J. Majda, and S. Schochet. A simple justification of the singular limit for equatorial shallow-water dynamics. Communications on Pure and Applied Mathematics, 62(3):322-333, 2009.

[134] I. Gallagher and L. Saint-Raymond. Mathematical study of the betaplane model: Equatorial 
waves and convergence results. Mémoires de la Société Mathématique de France, 107:116, 2006.

[135] A. Arakawa. The cumulus parameterization problem: Past, present, and future. J. Climate, 17(13):2493-2525, 2004.

[136] B. Khouider and A. J. Majda. A simple multicloud parametrization for convectively coupled tropical waves. Part II: Nonlinear simulations. J. Atmos. Sci., 64:381-400, 2007.

[137] W. W. Grabowski. MJO-like coherent structures: sensitivity simulations using the cloudresolving convection parameterization (CRCP). J. Atmos. Sci., 60:847-864, March 2003. 